\title{
SUR LA THÉORIE DU POTENTIEL DANS LES DOMAINES DE JOHN
}

\author{
Alano Ancona \\ Abstract \\ Using rather elementary and direct methods, we first recover and \\ add on some results of Aikawa-Hirata-Lundh about the Martin \\ boundary of a John domain. In particular we answer a question \\ raised by these authors. Some applications are given and the case \\ of more general second order elliptic operators is also investigated. \\ In the last parts of the paper two potential theoretic results are \\ shown in the framework of uniform domains or the framework of \\ hyperbolic manifolds.
}

\section{Introduction}

Dans leur travail récent [AHL], H. Aikawa, K. Hirata et T. Lundh ont étudié la compactification de Martin d'un domaine de John de $\mathbb{R}^{d}$. Ces auteurs ont en particulier établi —cf. [AHL, §6] — un principe de Harnack au bord "faible" (essentiellement l'énoncé du Théorème 3.1 ci dessous, à quelques modifications près) et ont montré que pour un tel domaine $\Omega$, l'ensemble des points minimaux sur la frontière de Martin de $\Omega$ qui sont associés (en un sens naturel, cf. [AHL]) à un point $\xi \in \partial \Omega$ donné est fini, de cardinal $N(\xi)$ majoré par un entier $N_{\Omega}$ ne dépendant que de $d$ et de la constante de John de $\Omega$ (voir un énoncé plus précis dans $[\mathbf{A H L}]$ ). Dans le présent article, on se propose d'abord (Sections 2 et 3) de retrouver par des méthodes directes élémentaires - proches de notre travail [An3] — l'inégalité de Harnack au bord susmentionnée de $[\mathbf{A H L}]$ puis de répondre positivement à une question laissée ouverte par ces auteurs : on a $N_{\xi} \leq m$ si $\xi \in \partial \Omega$ admet pour chaque $R>0$ assez petit un système de $m$ points dominants sur $\partial B(a, R)$ [voir la Section 1 pour les définitions]. Ce qui résulte suivant les méthodes de [An3] d'une inégalité de Harnack au bord "forte" basée sur ces points (inégalité conjecturée dans $[\mathbf{A H L}]$ ) et précise le lien entre la géométrie 
de $\Omega$ et sa compactification de Martin. La Section 4 est consacrée à la démonstration de cette inégalité qui est obtenue en étendant par une récurrence convenable l'argument déjà utilisé pour les domaines de Denjoy dans [An3] et en s'inspirant d'un travail de A. Lömker [Löm] sur la frontière de Martin des domaines multisectoriels. L'approche adoptée ici fournit en outre des énoncés souples et bien localisés (cf. les Sections 5 et 6 où sont décrites certaines conséquences des inégalités de Harnack au bord obtenues). La Section 6 donne en termes des arcs de John dans $\Omega$ plusieurs formulations utiles du principe de Harnack au bord pour le cas des domaines intérieurement uniformes.

Dans la Section 7, on examine le cas de la théorie du potentiel relative à un opérateur elliptique d'ordre 2 assez général. On montre que les résultats obtenus pour le Laplacien s'étendent à de tels opérateurs sous des hypothèses d'uniforme régularité des domaines envisagés en utilisant des résultats de [An7]. Nous ignorons ce qu'il advient lorsqu'on omet cette hypothèse - peut-être artificielle - et que l'opérateur n'est pas supposé formellement auto-adjoint.

Les Sections 8 et 9 sont consacrées à deux types de résultats pour le cas (très spécial) des domaines uniformes. Elles sont à ce titre un peu disjointes de ce qui précède.

Dans la Section 8, on établit, dans le sillage de $[\mathbf{D o o}],[\mathbf{L M T}]$ et $[\mathbf{A C}]$, une version du théorème de limite radiale de Littlewood pour les domaines (intérieurement) uniformes; ici un argument nouveau est introduit pour éviter l'hypothèse — requise dans [AC, §A.1]—que la densité capacitaire du complémentaire du domaine est strictement positive.

Dans la Section 9, on établit, en application d'une des formes du principe de Harnack au bord et pour le cadre d'un domaine intérieurement uniforme, ou pour celui d'un opérateur elliptique du second ordre sur une variété riemannienne complète hyperbolique, le caractère quasi-métrique de l'inverse du noyau de Naïm, résultats partiellement annoncés dans [An8, pp. 131-132] (cf. aussi [An9]) et répondant à une question de Kalton-Verbitsky [KV]. Indépendamment, W. Hansen a établi cette propriété de quasi-métrique pour un domaine uniforme [Han]. L'argument ici est basé plus explicitement sur des considérations de géométrie hyperboliques et est bien adapté au cadre de la théorie du potentiel sur une variété hyperbolique.

Remerciements. Je remercie Hiroaki Aikawa qui m'a transmis les versions préliminaires de $[\mathbf{A i k}],[\mathbf{A L M}],[\mathbf{A H L}]$ et m'a signalé le problème soulevé en [AHL, Remark 2.2 et Remark 6.1]. Je lui dois aussi une lecture attentive d'une première version de la preuve du principe de Harnack 
"fort" de la Section 4 et des commentaires sur cette preuve. Je remercie également le referee pour de nombreuses et très utiles remarques.

\section{Quelques définitions et lemmes élémentaires préliminaires}

1.A. Rappelons d'abord la notion d'arc de John [MS $]$ qui sera centrale dans la suite. Dans tout ce qui suit, on notera si $\Omega$ est un domaine de $\mathbb{R}^{d}$ et si $x \in \bar{\Omega}$ (adhérence de $\Omega$ dans $\mathbb{R}^{d}$ ), $\delta_{\Omega}(x)$ (ou même $\delta(x)$ s'il n'y a pas d'ambiguité sur $\Omega$ ) la distance $d(x, \partial \Omega)$.

Définition 1.1. Soit $\gamma:[\alpha, \beta] \rightarrow \bar{\Omega}$ un arc rectifiable dans $\bar{\Omega}$ et soit $c>0$. On dira que $\gamma$ est un $c$-arc de John de $\Omega$ (ou encore un $c$-arc de John unilatère dans $\Omega$ ) si

$$
\delta_{\Omega}(\gamma(t)) \geq c \operatorname{long}\left(\gamma_{\mid[\alpha, t]}\right) .
$$

On dira qu'un point $A$ de $\bar{\Omega}$ peut être joint à un point $B$ de $\Omega$ par un $c$-arc de John de $\Omega$, s'il existe un $c$-arc de John de $\Omega$ d'origine $A$ et d'extrémité $B$. Évidemment ici les rôles de $A$ et $B$ ne sont pas échangeables en général. Le mot unilatère pourra servir ici à souligner ce fait et à éviter toute confusion avec les arcs uniformes ou arcs de John bilatères dans $\Omega$ utilisés dans les Sections 6 et suivantes.

La reformulation élémentaire (et plutôt évidente) suivante des inégalités de Harnack en termes d'arc de John nous sera très utile. Soit $\gamma:[\alpha, \beta] \rightarrow \Omega$ un $c_{0}$-arc de John $\left(c_{0}>0\right)$ de l'ouvert $\Omega$ de $\mathbb{R}^{d}$, issu de $A \in \Omega$ et aboutissant à $B \in \Omega$. Soit aussi $\varepsilon_{0}>0$.

Proposition 1.2. Soit $P$ un point de $\mathbb{R}^{d}$ tel que $d(P, A) \geq \varepsilon_{0} \delta_{\Omega}(A)$, $d(P, B) \geq \varepsilon_{0} \delta_{\Omega}(B)$, soient $U=\left\{x \in \Omega ; d(x, \gamma)<\varepsilon_{0} \delta_{\Omega}(A)\right\}$ et $h$ une fonction harmonique positive sur $U \backslash\{P\}$. Il existe une constante $C=$ $C\left(c_{0}, \varepsilon_{0}, \frac{\delta_{\Omega}(B)}{\delta_{\Omega}(A)}, d\right) \geq 1$ telle que

$$
C^{-1} h(A) \leq h(B) \leq C h(A) .
$$

En particulier, la validité des inégalités de Harnack entre $A$ et $B$ n'est pas mise en cause par l'éventuelle proximité de $P$ et $\gamma$; le point $P$ peut même être un point de l'arc $\gamma$.

Démonstration: On peut supposer $\alpha=0, c_{0} \leq 1$, la paramétrisation $\gamma$ intrinsèque (i.e. $t=\operatorname{long}\left(\gamma_{[0, t]}\right)$ pour $0 \leq t \leq \beta$ ), et donc $\beta=\ell:=$ $\operatorname{long}(\gamma)>0$.

Pour tout $t \in[0, \ell]$, on a $d\left(\gamma(t), \Omega^{c}\right)>\frac{1}{2} c_{0} \delta_{\Omega}(A)$ (distinguer $t \leq$ $\frac{1}{2} \delta_{\Omega}(A)$ et $\left.t>\frac{1}{2} \delta_{\Omega}(A)\right)$. Donc, quitte à remplacer $\varepsilon_{0}$ par $\min \left\{\varepsilon_{0}, \frac{c_{0}}{2}\right\}$, on 
a $U=\left\{x \in \mathbb{R}^{d} ; d(x, \gamma)<\varepsilon_{0} \delta_{\Omega}(A)\right\}$ (i.e. $d(x, \gamma)<\varepsilon_{0} \delta_{\Omega}(A) \Rightarrow x \in \Omega$ ). Notons aussi que $\ell \leq c_{0}^{-1} \delta_{\Omega}(B)=L\left(c_{0}, \frac{\delta_{\Omega}(B)}{\delta_{\Omega}(A)}\right) \times \delta_{\Omega}(A)$.

Choisissons alors des points $t_{0}=0<\cdots<t_{N-1}<t_{N}=\ell$ de $[0, \ell]$ tels que $d\left(\gamma\left(t_{i}\right), \gamma\left(t_{i+1}\right)\right)=\frac{\varepsilon_{0}}{12} \delta_{\Omega}(A)$ pour $i \leq N-2$, et $d\left(\gamma\left(t_{N-1}\right), \gamma\left(t_{N}\right)\right) \leq$ $\frac{\varepsilon_{0}}{12} \delta_{\Omega}(A)$; évidemment $(N-1) \times \frac{\varepsilon_{0}}{12} \leq L$. Supprimant les $t_{j} \in\{1, \ldots, N-$ $1\}$ tels que $d\left(\gamma\left(t_{j}\right), P\right)<\frac{\varepsilon_{0}}{24} \delta_{\Omega}(A)$, on obtient une nouvelle subdivision $t_{0}^{\prime}<t_{1}^{\prime}<\cdots<t_{m}^{\prime}=\ell$ de $[0, \ell]$ telle que $m \leq m_{0}:=1+$ $\frac{12}{\varepsilon_{0}} L, d\left(\gamma\left(t_{i}^{\prime}\right), \gamma\left(t_{i+1}^{\prime}\right)\right) \leq \frac{\varepsilon_{0}}{4} \delta_{\Omega}(A)$ pour $0 \leq i<m$ et $d\left(\gamma\left(t_{i}^{\prime}\right), P\right) \geq$ $\frac{\varepsilon_{0}}{24} \delta_{\Omega}(A)$ pour $i \leq m$. Si $d\left(\gamma\left(t_{i}^{\prime}\right), P\right) \geq \frac{\varepsilon_{0}}{2} \delta_{\Omega}(A)$, les inégalités de Harnack ordinaires dans la boule $B\left(\gamma\left(t_{i}^{\prime}\right), \frac{\varepsilon_{0}}{2} \delta_{\Omega}(A)\right)$ donnent $c^{-1} h\left(\gamma\left(t_{i}^{\prime}\right)\right) \leq$ $h\left(\gamma\left(t_{i+1}^{\prime}\right)\right) \leq c h\left(\gamma\left(t_{i}^{\prime}\right)\right)$ avec $c=c(d)$. Si $d\left(\gamma\left(t_{i}^{\prime}\right), P\right)<\frac{\varepsilon_{0}}{2} \delta_{\Omega}(A)$, le lemme élémentaire suivant appliqué à la boule $B\left(P, \frac{\varepsilon_{0}}{2} \delta_{\Omega}(A)\right)$ donne $c^{-1} h\left(\gamma\left(t_{i}^{\prime}\right)\right) \leq h\left(\gamma\left(t_{i+1}^{\prime}\right)\right) \leq c h\left(\gamma\left(t_{i}^{\prime}\right)\right)$ avec $c=c(d)$. Comme $m \leq m_{0}$, on a bien $C^{-1} h(A) \leq h(B) \leq C h(A)$ avec $C=C\left(c_{0}, \varepsilon_{0}, \frac{\delta_{\Omega}(B)}{\delta_{\Omega}(A)}, d\right)$.

Lemme 1.3. Soit $h$ une fonction harmonique positive sur la boule épointée $B(0,1) \backslash\{0\}$ de $\mathbb{R}^{d}$, soient $\alpha, \beta \in B(0,1 / 2)$ tels que $|\alpha|,|\beta| \geq \varepsilon>0$. Alors $c^{-1} h(\alpha) \leq h(\beta) \leq c h(\alpha)$ pour un réel $c=c(d, \varepsilon) \geq 1$.

Remarquons que la Proposition 1.2 admet l'amélioration "automatique" suivante.

Proposition 1.2bis. Soient $U=\left\{x \in \Omega ; d(x, \gamma)<\varepsilon_{0} \delta_{\Omega}(A)\right\}$ et $s$ une fonction surharmonique positive sur $U$ qui est harmonique dans $V=$ $\left\{x \in U ; \min \{d(x, A), d(x, B)\}<\varepsilon_{0} \delta_{\Omega}(A)\right\}$. Il existe une constante $C=$ $C\left(c_{0}, \varepsilon_{0}, \frac{\delta_{\Omega}(B)}{\delta_{\Omega}(A)}, d\right) \geq 1$ telle que

$$
C^{-1} s(A) \leq s(B) \leq C s(A) .
$$

Par la représentation de Riesz de $s$ dans $U, s$ est somme d'une fonction $h$ harmonique positive dans $U$ et du potentiel $p()=.\int_{U \backslash V} G_{x}(). d \mu(x)$ où $G_{x}$ désigne la fonction de Green de pôle $x$ dans $U$ et où $\mu$ désigne la mesure $-\Delta s$. Appliquant la Proposition 1.2 à $h$ et chacune des $G_{x}$ on obtient la double inégalité voulue.

1.B. Passons maintenant à deux lemmes simples liés à l'existence d'arcs de John dans $\Omega$.

Lemme 1.4. Soient $\Omega$ un ouvert de $\mathbb{R}^{d}, a \in \mathbb{R}^{d}$ et $U$ une composante connexe de $B(a, R) \cap \Omega, R>0$. Supposons que pour un $c_{0}>0$ tout point de $B(a, R / 4) \cap U$ puisse être joint à un point de $\Omega \backslash U$ par un $c_{0}$-arc de John de $\Omega$. Alors, tout point $x$ de $B(a, R / 4) \cap U$ peut être joint à au moins un point de $U \cap \partial B(a, R / 2)$ par un $c_{0}$-arc de John de $U$. 
En effet, si l'arc rectifiable $\gamma:[0, \ell] \rightarrow \Omega$ joint $x \in B(a, R / 4) \cap U$ à $y \in \Omega \backslash U$ et est $c_{0}$-John dans $\Omega$, il existe $t_{0} \in(0, \ell]$ minimum tel que $\gamma\left(t_{0}\right) \in \partial B(a, R / 2)$ (sinon $\left.\gamma([0, \ell]) \subset U\right)$. Alors $\gamma_{0}=\gamma_{\left[0, t_{0}\right]}$ est tracé dans $U$ et pour $0 \leq t \leq t_{0}$, on a $B\left(\gamma(t), c_{0} t\right) \subset \Omega \cap B(a, R)$. Donc $B\left(\gamma(t), c_{0} t\right) \subset U$ et $\gamma_{0}$ est $c_{0}$-John dans $U$.

Lemme 1.5. Soient $\Omega$ un ouvert de $\mathbb{R}^{d}, a \in \mathbb{R}^{d}, R>0,0<\eta<1$, tels que chaque point $z \in B(a,(1-\eta) R) \cap \Omega$ puisse être joint à (au moins) un point de $\Omega \backslash B(a, R)$ par un $c_{0}$-arc de John dans $\Omega$ issu de $z\left(c_{0}>0\right)$. Il existe alors une famille finie $\left\{A_{j}\right\}_{1 \leq j \leq m}$ de points de $\partial B(a, R) \cap \Omega$ telle que: (i) on a $m \leq m_{0}$ pour un entier $m_{0}=m_{0}\left(d, c_{0}, \eta\right)$, (ii) $\delta_{\Omega}\left(A_{j}\right) \geq$ $\eta c_{0} R$ pour $1 \leq j \leq m$ et (iii) chaque $x \in B(a,(1-\eta) R) \cap \Omega$ peut être joint à l'un des $A_{j}$ par un $\frac{c_{0}}{2}$-arc de John dans $\Omega$.

Preuve: Voir aussi la très semblable Proposition 2.1 de [AHL]. Soit $K$ l'ensemble (qu'on peut supposer non vide) des traces sur $\partial B(a, R)$ des $c_{0}$-arcs de John joignant un point de $B(a,(1-\eta) R) \cap \Omega$ à un point dans $\Omega \backslash B(a, R)$. Par construction, pour chaque $z \in K$, on a $\delta_{\Omega}(z) \geq$ $\eta c_{0} R$. Soit $\left\{A_{j}\right\}_{1 \leq j \leq m}$ un ensemble maximal de points de $K$ tel que $d\left(A_{j}, A_{k}\right) \geq c_{0} \eta R / 4$ pour $j \neq k$. Les boules $B\left(A_{j}, \eta c_{0} \frac{R}{8}\right)$ étant deux à deux disjointes et contenues dans $B\left(a,\left(1+c_{0} \eta\right) R\right)$, on a $m \leq m_{0}:=$ $\left[\left(1+\eta c_{0}\right)^{d}\left(\frac{8}{\eta c_{0}}\right)^{d}\right]+1$.

D'après les hypothèses, un point donné $x \in B(a,(1-\eta) R) \cap \Omega$ peut être joint à au moins un point $y \in K$ par un $c_{0}$-arc de John $\gamma$ de $\Omega$; comme $d(y ; \partial \Omega) \geq c_{0} \eta R$ un calcul simple montre que choisissant $j$ tel que $d\left(y, A_{j}\right) \leq \frac{c_{0} \eta}{4} R$, l'arc $\tilde{\gamma}$ obtenu en complétant $\gamma$ par le segment orienté $\left[y, A_{j}\right]$ est $\frac{c_{0}}{2}$-John dans $\Omega$. D'où l'énoncé.

On voit d'ailleurs qu'on peut aussi dans le Lemme 1.5 imposer $d\left(A_{j}, A_{k}\right) \geq \frac{c_{0} \eta}{4} R$ pour $j \neq k$. Dégageons pour finir cette partie la notion suivante (très proche de la Définition 2.1 de $[\mathbf{A H L}]$ ) suggérée par le Lemme 1.5.

Définition 1.6. Soient $U \subset \Omega,\left\{A_{j}\right\}_{1 \leq j \leq m}$ un ensemble fini de points de $\Omega$ tel que chaque point $z \in U$ puisse être joint à (au moins) un des $A_{j}$ par un $c_{0}$-arc de John dans $\Omega$ issu de $z, c_{0}>0$. On dira alors que $\left\{A_{j}\right\}_{1 \leq j \leq m}$ est $c_{0}$-dominant pour $U$ dans $\Omega$.

1.C. Soit $\Omega$ un domaine de $\mathbb{R}^{d}$ et soit $\tilde{\partial} \Omega=\tilde{\Omega} \backslash \Omega$ la frontière de $\Omega$ vis à vis d'une compactification $\tilde{\Omega}$ de $\Omega$. Il sera utile de disposer d'une notion adéquate d'annulation au bord (cf. [An3, p. 221] ou [AHL] pour une notion parallèle mais distincte pour le cas où $\tilde{\Omega}$ est l'adhérence dans $\mathbb{R}^{d} \cup$ $\{\infty\})$. Cette notion n'interviendra pas avant la Section 5 . 
Définition 1.7. Soit $u$ une fonction sur $\Omega$ et soit $A \subset \tilde{\partial} \Omega$. On dira que $u$ s'annulle sur $A$ (au sens intrinsèque et relativement à la compactification $\tilde{\Omega}$ ) s'il existe $s$ surharmonique positive sur $\Omega$ (distincte de la constante $+\infty$ ) telle que $|u|=o(s)$ le long du filtre des (traces sur $\Omega$ ) des voisinages de $A$. On écrira alors $u=0$ sur $A$.

\section{Remarques 1.8 .}

1. (Principe du minimum). Si $u$ est sous-harmonique sur $\Omega$ telle que $u_{+}$s'annulle (au sens intrinsèque) sur $\tilde{\partial} \Omega$ alors $u \leq 0$ sur $\Omega$ (car $u \leq \varepsilon s$ pour tout $\varepsilon>0)$.

2. Si $u$ est bornée au voisinage de $A \subset \tilde{\partial} \Omega$ et si $A$ est de mesure harmonique nulle dans $\Omega$ (i.e. l'enveloppe de Perron supérieure $\bar{H}_{1_{A}}$ est nulle) alors $u=0$ sur $A$.

3. Si $u$ est nulle sur $A_{j} \subset \tilde{\partial} \Omega, j=1,2$, alors $u=0$ sur $A_{1} \cup A_{2}$.

4. Soit $u \geq 0$ sur le domaine $\Omega$ et soit $x_{0} \in \Omega$ fixé. Pour que $u$ soit nulle sur $A \subset \tilde{\partial} \Omega$ il suffit que pour tout $\varepsilon>0$ on ait $u \leq s+p$ au voisinage de $A$, pour une fonction $s$ surharmonique positive dans $\Omega$ telle que $s\left(x_{0}\right) \leq \varepsilon$ et un potentiel $p$ sur $\Omega$ (i.e. $p$ est surharmonique dans $\Omega$ et la plus grande minorante harmonique de $p$ dans $\Omega$ est nulle, [Br4]) : en effet, en remplaçant $p$ par une réduite (ref. [Br4], [Her]) $\widehat{R}_{p}^{\Omega \backslash K}$ dans $\Omega$, avec $K$ compact assez grand dans $\Omega$, on voit qu'existe $s^{\prime}$ surharmonique $\geq 0$ dans $\Omega$ telle que $u \leq s^{\prime}$ au voisinage de $\tilde{\partial} \Omega$ et $s^{\prime}\left(x_{0}\right) \leq 2 \varepsilon$. Prenant alors $s_{n}^{\prime}$ associée à $\varepsilon_{n}=2^{-n}$ on voit que $\sigma=\sum_{n \geq 1} s_{n}^{\prime}$ est surharmonique $(\sigma \not \equiv+\infty)$ dans $\Omega$ et que $u=o(\sigma)$ le long du filtre des voisinages de $A$.

5. En particulier tout potentiel s'annulle sur $\partial \widetilde{\Omega}$. Inversement si $s$ est surharmonique positive, nulle au sens intrinsèque sur $\tilde{\partial} \Omega$, alors $s$ est un potentiel. Cela se déduit aussitôt du principe du minimum du 1.8.1 ci-dessus.

Exemples 1.9. (a) Si on prend $\tilde{\Omega}=\bar{\Omega}$ (l'adhérence ordinaire dans $\mathbb{R}^{d} \cup$ $\{\infty\})$, si $u: \Omega \rightarrow \overline{\mathbb{R}}_{+}$est bornée au voisinage de $A \subset \partial \Omega$ et si $u$ tend vers zéro en tout point $\xi \in A$ hors d'un ensemble polaire, alors $u=0 \operatorname{sur} A$. On retrouve ici la notion d'annulation au bord utilisée dans $[\mathbf{A n 3}],[\mathbf{A i k}],[\mathbf{A L M}]$.

(b) Si $s$ surharmonique positive sur $\Omega$ est nulle sur la partie $A$ de $\tilde{\partial} \Omega$, si $F \subset \Omega$ est tel que $\bar{F} \cap \tilde{\partial} \Omega \subset A$, alors la réduite régularisée $u=\widehat{R}_{s}^{F}$ de $s$ sur $F$ dans $\Omega$ est un potentiel (dans $\Omega$ ). En effet, si $\sigma$ est surharmonique positive sur $\Omega$ telle que $s=o(\sigma)$ au voisinage de 
$A$ dans $\Omega$, si - pour $\varepsilon>0$ fixé- $L$ est l'ensemble (relativement compact dans $\Omega) L:=\{x \in F ; s \geq \varepsilon \sigma\}$, on a

$$
\widehat{R}_{s}^{F} \leq \widehat{R}_{s}^{F \backslash L}+\widehat{R}_{s}^{L} \leq \varepsilon \sigma+\widehat{R}_{s}^{L}
$$

où $\widehat{R}_{s}^{L}$ est un potentiel puisque $L$ est relativement compact dans $\Omega$. On en déduit par le 1.8.4 ci-dessus que $\widehat{R}_{s}^{F}$ est nul (au sens intrinsèque) sur $\partial \Omega$. Le 1.8.5 dit alors que $\widehat{R}_{s}^{F}$ est un potentiel.

\section{Un premier contrôle de la fonction de Green}

Cette partie est consacrée à la preuve du Théorème 2.1 suivant. La majoration qui y apparait est l'analogue du Lemme 5.3 de [AHL]. Elle étend le Lemme 7.1 de [An3] dont on reprend le mode de démonstration. Ce type d'estimation trouve son origine dans $[\mathbf{C a r}]$ et $[\mathbf{H W} 1],[\mathbf{H W 2}]$. Voir aussi [Tay], [An1], [An2], [CFMS], [JK].

Soient $a \in \mathbb{R}^{d}$ et $\Omega$ un ouvert de Green de $\mathbb{R}^{d}$ dont la fonction de Green sera notée $G$. On notera aussi $G(x, y)=G_{y}(x)$ pour $(x, y) \in \Omega$.

Théorème 2.1. Soient $R>0, c_{0}>0$, et soient $A_{1}, \ldots, A_{m}$ des points de $\partial B(a, R) \cap \Omega$ tels que chaque point $z \in B\left(a, \frac{1}{2} R\right) \cap \Omega$ puisse être joint à au moins l'un des $A_{j}$ par un $c_{0}$-arc de John dans $\Omega$ issu de $z$. On a alors

$$
G_{x}(y) \leq C \sum_{j=1}^{m} G_{x}\left(A_{j}\right)
$$

pour tout $x \in \partial B\left(a, \frac{1}{4} R\right) \cap \Omega$ et tout $y \in \partial B\left(a, \frac{1}{2} R\right) \cap \Omega$. Ici $C=$ $C\left(d, c_{0}\right)>0$

L'hypothèse est donc que $\left\{A_{j}\right\}_{1 \leq j \leq m}$ est $c_{0}$-dominant pour $\Omega \cap B\left(a, \frac{1}{2} R\right)$ dans $\Omega$ [cf. la Définition 1.6]. Dans les paragraphes 2.A et suivants on verra des formes plus générales de (2.1) mais qui en résultent facilement.

Remarques 2.2. (1) Par le principe du maximum, l'inégalité (2.1) s'étend aux $y \in \Omega \backslash B\left(a, \frac{R}{2}\right)$ et aux $x \in B\left(a, \frac{R}{4}\right) \cap \Omega$.

(2) Quitte à remplacer $c_{0}$ par $c_{0}^{\prime}=\frac{1}{2} c_{0}$, on peut aussi après suppression de certains des $A_{j}$ supposer que $d\left(A_{j}, A_{k}\right) \geq c_{0} \frac{R}{8}$ pour $j \neq k$ et donc $m \leq m_{0}(c, d)$ : si $J \subset\{1, \ldots, m\}$ est maximal tel que $d\left(A_{j}, A_{k}\right) \geq c_{0} \frac{R}{8}$ pour $j, k \in J, j \neq k$, alors $\left\{A_{j}\right\}_{j \in J}$ convient. [Cf. la fin de la preuve du Lemme 1.5.]

Pour établir le Théorème 2.1, on va passer par un énoncé plus technique. 
Proposition 2.3. Soient $R>0, a \in \partial \Omega, c_{0}>0, m_{0} \geq 1$ et soit pour chaque $n \geq 0$, une famille $\left\{A_{j}^{n}\right\}_{1 \leq j \leq m_{0}}$ de points de $\partial B\left(a, 2^{-n} R\right)$ $c_{0}$-dominante pour $\Omega \cap B\left(a, 2^{-n-1} R\right)$ dans $\Omega$. Alors pour $x \in \Omega$ tel que $\delta_{\Omega}(x)=d(x, a)$ et $0<\delta_{\Omega}(x) \leq \frac{R}{4}$, on $a$

$$
G_{x}(y) \leq C \sum_{j=1}^{m_{0}} G_{x}\left(A_{j}\right)
$$

pour tout $y \in \partial B\left(a, \frac{1}{2} R\right) \cap \Omega$. Ici $A_{j}=A_{j}^{0}$ et $C=C\left(d, c_{0}\right)>0$.

Preuve de la Proposition 2.3: (a) Observons d'abord la majoration plus faible suivante : pour $x \in \Omega$ tel que $\frac{R}{2^{n+1}} \leq \delta(x) \leq \frac{R}{2^{n}}, n \geq 2$, et $\delta(x)=d(x, a)$, on a

$$
G_{x}(y) \leq C_{n} \sum_{j=1}^{m_{0}} G_{x}\left(A_{j}\right)
$$

pour tout $y \in \partial B\left(a, \frac{1}{2} R\right) \cap \Omega$ avec une constante $C_{n}=C_{n}\left(d, c_{0}\right)$ dépendant de $n, d$ et $c_{0}$.

En utilisant l'existence d'un $c_{0}$-arc de John dans $\Omega$, issu de $x$ et d'extrémité $A_{j_{0}}$ (pour un $j_{0}$ convenable), et à l'aide des inégalités de Harnack de la Proposition 1.2 (appliquée à la fonction $x \mapsto G_{x}(y)$ qui est harmonique sur $\Omega \backslash\{y\}$ ), on obtient

$$
G_{x}(y) \leq C_{n}^{1} G_{A_{j_{0}}}(y),
$$

avec $C_{n}^{1}=C_{n}^{1}\left(d, c_{0}\right)$. Par le principe du maximum, on a $G_{A_{j_{0}}}(y) \leq$ $\sup \left\{G_{A_{j_{0}}}(z) ; d\left(A_{j_{0}}, z\right)=\frac{c_{0}}{10} R\right\}$. Une nouvelle utilisation des inégalités de Harnack de la Proposition 1.2 nous donne à partir de là une constante $C_{n}^{2}$-dépendant de $n, d$ et $c_{0}$ - telle que

$$
G_{A_{j_{0}}}(y) \leq C_{n}^{2} G_{x}\left(A_{j_{0}}\right)
$$

et donc (2.3) avec $C_{n}=C_{n}^{1} C_{n}^{2}$.

(b) Notons $\widetilde{C}_{n}=\widetilde{C}_{n}\left(d, c_{0}\right)$ la plus petite constante $C_{n}$ ne dépendant que de $n, c_{0}$ et $d$ telle qu'on ait la majoration (2.3) chaque fois que les hypothèses de la Proposition 2.3 ont lieu. On a en particulier sous les hypothèses de la Proposition 2.3,

$$
G_{x}(y) \leq \widetilde{C}_{n} \sum_{j=1}^{m_{0}} G_{x}\left(A_{j}^{p}\right)
$$


pour $n \geq 2, p \geq 0,2^{-p-n-1} R \leq \delta(x)=d(x, a) \leq 2^{-p-n} R$, et $y \in$ $\partial B\left(a, 2^{-p-1} R\right.$ ) (en remplaçant $R$ par $2^{-p} R$ à $p$ fixé et en considérant les systèmes de points $\left.\left\{A_{j}^{p+q}\right\}_{1 \leq j \leq m_{0}}, q \geq 0\right)$.

(c) Il suffira pour établir la Proposition 2.3 de montrer que les $\widetilde{C}_{n}$ sont bornés uniformément par rapport à $n$. A cette fin, on adapte la méthode du Lemme 7.1 de [An3] qui porte sur les domaines de Denjoy lipschitziens.

Si $n \geq 3$, on a pour $\frac{1}{2^{n+2}} R \leq \delta(x) \leq \frac{1}{2^{n+1}} R$ et par définition de $\widetilde{C_{n}}$ la majoration

$$
G_{x}(y) \leq \widetilde{C}_{n} \sum_{j=1}^{m_{0}} G_{x}\left(A_{j}^{1}\right)
$$

pour $y \in \Omega \backslash B\left(a, \frac{1}{4} R\right)$.

Joignant chacun des $A_{j}^{1}$ à l'un des $A_{k}^{0}$ par un $c_{0}$-arc de John dans $\Omega$, une utilisation des inégalités de Harnack (sous la forme de la Proposition 1.2) nous donne une constante $c^{\prime}=c\left(c_{0}, d\right)$ telle que

$$
G_{x}(z) \leq c^{\prime} \tilde{C}_{n} \sum_{j=1}^{m_{0}} G_{x}\left(A_{j}^{0}\right)
$$

pour tout $z \in \Omega \backslash B\left(a, \frac{1}{4} R\right)$.

Prenons maintenant $y \in \partial B\left(a, \frac{1}{2} R\right) \cap \Omega$ et un $c_{0}$-arc de John $\gamma:[0, \ell] \rightarrow$ $\Omega$ (en paramétrisation intrinsèque) joignant $y$ à l'un des $A_{j}^{0}$, soit $A_{j_{0}}^{0}$; on a donc $\alpha^{-1} R \leq \ell \leq \alpha R, \alpha=\alpha\left(c_{0}\right)$. Considérons ensuite pour un $\eta>0$ petit qu'on fixera ultérieurement, l'ensemble fermé (pseudo tronc de cône de sommet $\gamma(\eta \ell)$ et d' "axe" $\gamma_{\mid[\eta \ell, \ell]}$ — c'est une "carotte" dans la terminologie de [Va1], [NV]-,

$$
F=\bigcup_{\eta \ell \leq t \leq \ell}\left\{\bar{B}\left(\gamma(t), \frac{c_{0}}{4}(t-\eta \ell)\right)\right\} \subset \Omega .
$$

Notons $y_{\eta}=\gamma(\eta \ell)$ le "sommet" de $F$ et introduisons l'ouvert $U=$ $\Omega \cap\left(B\left(y_{\eta}, \frac{R}{10}\right) \backslash F\right)$. Si $h_{1}$ est la solution du problème de Dirichlet dans $U$ pour la donnée frontière $1_{F \cap \partial U} G_{x}$, et si $h_{2}$ est celle correspondant à la donnée frontière $1_{\Omega \cap \partial B(y, R / 10) \backslash F} G_{x}$, les $h_{j}$ sont harmoniques positives dans $U$ et $G_{x}=h_{1}+h_{2}$ dans $U$ puisque $G_{x}=0$ sur $\partial \Omega$.

Par la définition de $h_{2}$, le principe du maximum et l'inégalité (2.7) ci-dessus, on a

$$
h_{2}(y) \leq c^{\prime} \widetilde{C}_{n}\left(\sum_{j=1}^{m_{0}} G_{x}\left(A_{j}^{0}\right)\right) \mu_{y}\left(\partial B\left(y_{\eta}, \frac{R}{10}\right) \backslash F\right)
$$


où $\mu_{y}\left(\partial B\left(y_{\eta}, \frac{R}{10}\right) \backslash F\right)$ est la mesure harmonique en $y$ de $\partial B\left(y_{\eta}, \frac{R}{10}\right) \backslash F$ dans $B\left(y_{\eta}, \frac{R}{10}\right) \backslash F$ (elle vaut 0 si $y \in F$ ). Par le Lemme 2.4 ci-dessous on a $\mu_{y}\left(\partial B\left(y_{\eta}, \frac{R}{10}\right) \backslash F\right) \leq \frac{1}{2 c^{\prime}}$ si on choisit $\eta=\eta\left(c^{\prime}, c_{0}, d\right)$ assez petit, choix (indépendant de $n$ ) qu'on fixe désormais. Alors,

$$
h_{2}(y) \leq \frac{1}{2} \widetilde{C}_{n}\left(\sum_{j=1}^{m_{0}} G_{x}\left(A_{j}\right)\right) .
$$

D'autre part pour tout $z \in \partial F$, les inégalités de Harnack et la Proposition 1.2 nous donnent (une fois $\eta>0$ fixé comme ci-dessus) que $G_{x}(z) \leq$ $c^{\prime \prime} G_{x}\left(A_{j_{0}}\right)$ pour tout $z \in \partial F \cap B\left(y, \frac{R}{10}\right)$ avec $c^{\prime \prime}=c^{\prime \prime}\left(c_{0}, \eta, d\right)>0$. Et $h_{1}(y) \leq c^{\prime \prime} G_{x}\left(A_{j_{0}}\right)$.

On aboutit ainsi à la majoration : $\widetilde{C}_{n+1} \leq \frac{1}{2} \widetilde{C}_{n}+c^{\prime \prime}$ pour tout $n \geq 0$. Mais alors $\widetilde{C}_{n+1} \leq \max \left\{\widetilde{C}_{n}, 2 c^{\prime \prime}\right\}$ et donc $\widetilde{C}_{n} \leq K:=\max \left\{C_{2}, 2 c^{\prime \prime}\right\}$. Ce qui achève d'établir la Proposition 2.3.

On a utilisé, comme dans [An3] (cf. le Lemme 6.3), le lemme bien classique suivant. Une preuve est indiquée pour la commodité du lecteur.

Lemme 2.4. Soient $c \in\left(0, \frac{1}{4}\right)$ et $F$ un fermé de la boule ouverte $B(0,1)$. Supposons que pour tout $n \geq 1$ il existe $x_{n}$ tel que $\left|x_{n}\right|=\frac{1}{2^{n}}$ et $B\left(x_{n}, c 2^{-n}\right) \subset$ $F$. Alors la mesure harmonique $\mu$ de $\partial B(0,1)$ dans $B(0,1) \backslash F$ vérifie

$$
\mu(x) \leq C\|x\|^{\alpha} \text { pour } x \in B(0,1)
$$

où $\alpha=\alpha(d, c) \in(0,1)$ et $C=C(d, c)>0$.

Preuve abrégée: On observe d'abord que la mesure harmonique $\nu$ de $\partial B(0,1)$ dans $\omega=B(0,1) \backslash B\left(m_{0}, \frac{c}{2}\right), m_{0} \in \partial B\left(0, \frac{1}{2}\right)$ vérifie $\nu(x) \leq(1-\varepsilon)$ pour $x \in B\left(0, \frac{1}{2}\right)$. Ici $\varepsilon=\varepsilon(d, c)>0$.

Posons $\mu_{n}:=\sup \left\{\mu(z) ;|z|=2^{-n}\right\}$. En combinant l'observation précédente et le principe du maximum on obtient que $\mu_{n+1} \leq(1-\varepsilon) \mu_{n}$. D'où $\mu_{n} \leq(1-\varepsilon)^{n}=2^{-n \alpha}$ où $\alpha=:-\log (1-\varepsilon)$ et le résultat : $\mu(x) \leq$ $2^{\alpha}\|x\|^{\alpha}$.

On déduit maintenant de ce qui précède le Théorème 2.1.

Preuve du Théorème 2.1: (a) Prenons $x \in \partial B(a, R / 4)$ et supposons d'abord que $\delta_{\Omega}(x) \leq \frac{R}{100}$. Soit $a^{\prime} \in \partial \Omega$ tel que $\delta(x)=d\left(x, a^{\prime}\right)$. Par le Lemme 1.5, il existe pour chaque $R^{\prime} \leq \frac{R}{25}$ des points $A_{j}^{\prime}\left(R^{\prime}\right)$ sur $\partial B\left(a^{\prime}, R^{\prime}\right)$, $1 \leq j \leq m_{0}=m_{0}\left(c_{0}, d\right)$, tels que de tout point $z \in \Omega \cap B\left(a^{\prime}, \frac{R^{\prime}}{2}\right)$ on puisse faire partir un $\frac{c_{0}}{2}$-arc de John de $\Omega$ d'extrémité l'un des $A_{j}^{\prime}\left(R^{\prime}\right)$. Par la 
Proposition 2.3 et le Lemme 1.5, on a alors pour tout $y \in \Omega \backslash B\left(a^{\prime}, \frac{R}{50}\right)$,

$$
G_{x}(y) \leq C \sum_{j=1}^{m_{0}} G_{x}\left(A_{j}^{\prime}\right) \leq c C \sum_{j=1}^{m} G_{x}\left(A_{j}\right)
$$

si $A_{j}^{\prime}=A_{j}^{\prime}\left(\frac{R}{25}\right)$. La deuxième inégalité vient des inégalités de Harnack de la Proposition 1.2 : à l'aide de $c_{0}$-arcs de John dans $\Omega$ joignant chaque $A_{j}^{\prime}$ à l'un des $A_{k}$, soit $A_{j_{k}}$, on trouve que $G_{x}\left(A_{j}^{\prime}\right) \leq c_{1} \sum_{1 \leq k \leq m} G_{x}\left(A_{j_{k}}\right)$ et $\operatorname{donc} \sum_{j=1}^{m_{0}} G_{x}\left(A_{j}^{\prime}\right) \leq m_{0} c_{1} \sum_{j=1}^{m} G_{x}\left(A_{j}\right), c_{1}=c_{1}\left(d, c_{0}\right)$.

(b) Reste à considérer le cas de $x \in B\left(a, \frac{R}{4}\right)$ tel que $\delta(x) \geq \frac{R}{100}$. Ici à l'aide d'un arc de John $\gamma$ joignant $x$ à l'un des $A_{j}$, soit $A_{j_{0}}$, et à l'aide des inégalités de Harnack (en particulier celles de la Proposition 1.2) on obtient

$$
G_{x}(y) \leq \sup \left\{G_{x}(z) ; d(x, z)=\frac{R}{200}\right\} \leq c G_{x}\left(z^{\prime}\right) \leq c c^{\prime} G_{x}\left(A_{j_{0}}\right)
$$

où $z^{\prime}$ est un point de $\gamma$ appartenant à $\partial B\left(x, \frac{R}{200}\right)$ et $c=c(d), c^{\prime}=$ $c^{\prime}\left(d, c_{0}\right)$. Ce qui donne l'inégalité du Théorème 2.1 .

2.A. Extension du Théorème 2.1. On peut facilement déduire du Théorème 2.1 une extension à la fois plus souple et plus satisfaisante. Donnons nous une partie fermée $F$ de $\Omega$ et un système $\left\{A_{j}\right\}_{1 \leq j \leq m}$ de points de $\Omega$ qui est $c_{0}$-dominant pour $F_{\rho}:=\{x \in \Omega ; d(x, F) \leq \rho\}$ dans $\Omega$ et tel que $c_{1} \rho \leq \delta_{\Omega}\left(A_{j}\right) \leq c_{1}^{-1} \rho$ pour $1 \leq j \leq m^{(1)}$. Alors pour $x \in \Omega \backslash F_{\rho}$ et $y \in F$ on a

$$
G_{x}(y) \leq C \sum_{1 \leq j \leq m} G_{x}\left(A_{j}\right)
$$

pour une constante $C=C\left(d, c_{0}, c_{1}\right)>0$.

Notons $\rho^{\prime}=\frac{1}{2} \rho$. D'après le principe du maximum il suffira d'établir l'inégalité (2.9) pour les $x \in \partial F_{\rho^{\prime}} \cap \Omega$. On peut aussi supposer que $d\left(x, A_{k}\right) \geq \frac{1}{2} c_{1} \rho$ pour $1 \leq k \leq m$. Par le Lemme 1.5, il existe alors une famille $\left\{A_{j}^{\prime}\right\}_{1 \leq j \leq m_{0}}$ de points de $\partial B\left(x, \frac{\rho}{10}\right) \cap \Omega$ qui est $\frac{c_{0}}{2}$-dominante pour $\Omega \cap B\left(x, \frac{\rho}{20}\right)$ dans $\Omega, m_{0}=m_{0}\left(d, c_{0}\right)$, et telle que $\delta\left(A_{j}^{\prime}\right) \geq \frac{c_{0} \rho}{20}$. D'après le Théorème 2.1 , on a $G_{x}(y) \leq C \sum_{j=1}^{m_{0}} G_{x}\left(A_{j}^{\prime}\right), c=c\left(d, c_{0}\right)>0$. Mais pour chaque $j$, l'utilisation d'un $c_{0}$-arc de John $\hat{A}_{j}^{\prime} \widehat{A}_{k_{j}}$ et la Proposition 1.2

\footnotetext{
(1) Alors chaque point $x \in F$ (et même chaque $x \in F_{\rho}$ ) est à distance $d\left(x, A_{j}\right)$ majorée par $c_{0}^{-1} c_{1}^{-1} \rho$ d'un des $A_{j}$.
} 
nous donnent que $G_{x}\left(A_{j}^{\prime}\right) \leq c^{\prime} G_{x}\left(A_{k_{j}}\right), c^{\prime}=c^{\prime}\left(d, c_{0}, c_{1}\right), 1 \leq j \leq m_{0}$ (noter qu'on peut supposer que $d\left(x, A_{k}\right) \geq \frac{c_{1} \rho}{2}$ pour $1 \leq k \leq m$ ). D'où la conclusion voulue.

2.B. Notons aussi une forme duale de (2.9). Sous les mêmes hypothèses qu'en 2.A ci-dessus, on a

$$
G_{x}(y) \leq C \sum_{j=1}^{m} G_{A_{j}}(y)
$$

pour une constante $C=C\left(d, c_{0}, c_{1}\right)>0$. Pour le voir on pourra observer d'abord qu'il suffit d'après le principe du maximum d'établir (2.10) pour $y \in \Omega \cap \partial F_{\rho^{\prime}}, \rho^{\prime}=\frac{1}{2} \rho$. Pour la même raison on peut aussi supposer $x \in \partial F_{\rho^{\prime \prime}}, \rho^{\prime \prime}=\frac{3}{4} \rho$. Mais, pour $x$ et $y$ de cette forme, on a

$$
G_{x}(y)=G_{y}(x) \leq C \sum_{1 \leq j \leq m} G_{y}\left(A_{j}\right)
$$

d'après le résultat du 2.A, en y substituant $\Omega \cap \partial F_{\rho^{\prime \prime}}$ à $F$ et $\frac{\rho}{4}$ à $\rho$ (permuter aussi les rôles de $x$ et $y$ ).

On peut enfin observer que dans les hypothèses du 2.A sur $F$ et les $A_{j}$, et si $U$ et $V$ sont deux composantes connexes distinctes de $\Omega \backslash F_{\rho}$, on a encore en application immédiate de (2.9)

$$
G_{x}(y) \leq C \sum_{1 \leq j \leq m} G_{x}\left(A_{j}\right)
$$

pour $x \in U$ et $y \in V$ avec $C=C\left(d, c_{0}, c_{1}\right)>0$.

\section{Le principe de Harnack au bord dans $\Omega$. Une forme très faible}

On montre maintenant - en adaptant une méthode bien connue ([An1, pp. 189-190], cf. aussi [An2], [CFMS], [JK], et la Proposition 7.2 de [An3] très voisine de la suivante) - que les majorations de Théorème 2.1 conduisent presqu'automatiquement à des majorations bien meilleures du point de vue du comportement au bord.

Notons aussi que le théorème suivant est à peu de choses près le Lemma 6.2 de [AHL] qu'on obtient donc ici par une autre voie. On désigne toujours par $G$ la fonction de Green d'un domaine greenien $\Omega$ de $\mathbb{R}^{d}$. 
Théorème 3.1. Soient $R$ un réel $>0, a \in \mathbb{R}^{d}$ et soient $A_{1}, \ldots, A_{m}$ (où $m \geq 1$ ) des points de $\partial B(a, R) \cap \Omega$ tels que chaque $z \in B\left(a, \frac{3}{4} R\right) \cap \Omega$ puisse être joint à au moins l'un des $A_{j}$ par un $c_{0}$-arc de John de $\Omega$ issu de z. On a alors

$$
G_{x}(y) \leq C R^{d-2} \sum_{1 \leq j, k \leq m} G_{x}\left(A_{j}\right) G_{A_{k}}(y)
$$

pour tout $x \in \partial B\left(a, \frac{1}{8} R\right) \cap \Omega$ et tout $y \in \partial B\left(a, \frac{1}{2} R\right) \cap \Omega$. Ici $C=$ $C\left(d, c_{0}\right)>0$.

Remarques. 1) Ici $x$ et $y$ jouent des rôles plus symétriques que dans les inégalités de la Section 2. Remarquons qu'ici encore, par le principe du maximum, les inégalités se prolongent au cas où $x \in$ $B\left(a, \frac{1}{8} R\right) \cap \Omega$ et où $y \in \Omega \backslash B\left(a, \frac{1}{2} R\right)$.

2) Pour $d=2$ le résultat obtenu sera en réalité un peu plus précis que l'énoncé ci-dessus. (Cf. le 3.2.)

Preuve: Par le Théorème 2.1 (cf. le 2.A) et le principe du maximum, on a pour $y \in \Omega \backslash \bar{B}\left(a, \frac{R}{4}\right)$

$$
G_{x}(y) \leq c\left[\sum_{k=1}^{m} G_{x}\left(A_{k}\right)\right] \mu(y)
$$

où $\mu$ désigne la mesure harmonique de $\partial B\left(a, \frac{R}{4}\right) \cap \Omega$ dans $V=\Omega \backslash \bar{B}\left(a, \frac{R}{4}\right)$ et $c=c\left(d, c_{0}\right)$ une constante positive. Or, si $g_{y}$ est la fonction de Green du point $y$ dans $V$ - prolongée par zéro hors de $V$-, le Laplacien au sens des distributions $\Delta g_{y}$ de $g_{y}$ coïncide avec la mesure harmonique de $y$ dans $V$ diminuée de la masse de Dirac en $y$. Donc $\mu(y)=\Delta g_{y}(\partial B(a, R / 4))$ et pour $d(y, a) \geq \frac{R}{2}$

(*) $\mu(y) \leq \int \Delta \varphi(z) g_{y}(z) d z \leq \int|\Delta \varphi(z)| g_{y}(z) d z \leq \int|\Delta \varphi(z)| G_{z}(y) d z$

si $\varphi \in C_{0}^{\infty}\left(\mathbb{R}^{d}\right)$ est positive, égale à 1 sur $\partial B(a, R / 4)$ et de support inclus dans $B\left(a, \frac{3 R}{8}\right)$. Avec le choix $\varphi(x)=\Phi\left(\frac{x-a}{R}\right)$ ( $\Phi$ correspondant au cas $a=0, R=1)$, il vient

$$
\mu(y) \leq C R^{d-2} \sup _{z \in B\left(a, \frac{3 R}{8}\right)} G_{z}(y) .
$$

Mais d'après le Théorème 2.1 (cf. le 2.B) et puisque $y \in \partial B\left(a, \frac{R}{2}\right)$, on a pour $z \in B\left(a, \frac{r}{8}\right)$,

$$
G_{z}(y)=G_{y}(z) \leq c \sum_{1 \leq j \leq m} G_{A_{j}}(y)
$$

où $c=c\left(d, c_{0}\right)$. Et la proposition s'ensuit. 
Remarques 3.2. Quitte à supprimer les $A_{j}$ "inutiles" (i.e. qui ne sont $c_{0}$-connectés à aucun $\left.x \in \Omega \cap B\left(a, \frac{3 R}{4}\right)\right)$ on peut supposer $\delta\left(A_{j}\right) \geq c_{0} \frac{R}{4}$ pour $j=1, \ldots, m$. De plus comme dans le Lemme 1.5 , quitte à encore supprimer des $A_{j}$, on peut supposer $m \leq m_{0}\left(d, c_{0}\right)$. Alors :

(i) $\operatorname{Si} d \geq 3$, on a $C_{1}^{-1} \leq R^{d-2} G_{A_{j}}(z) \leq C_{1}$ sur $\partial B\left(A_{j}, \frac{c_{0}}{8} R\right)$ pour tout $j$, avec $C_{1}=C_{1}(d)$; donc hors de $B_{j}=\bar{B}\left(A_{j}, \frac{c_{0}}{8} R\right), R^{d-2} G_{A_{j}}$ est équivalent en taille (avec des constantes ne dépendant que de $d$ ) au potentiel capacitaire $\pi_{j}:=R_{1}^{B_{j}}$-où la réduite est relative à $\Omega$ - de la boule $B_{j}$ dans $\Omega\left(\pi_{j}\right.$ est aussi la mesure harmonique de $B_{j}$ dans $\left.\Omega \backslash B_{j}\right)$. Et pour $x, y$ comme dans l'énoncé, on a la majoration (où $C=C\left(d, c_{0}\right)$ )

$$
G_{x}(y) \leq C \sum_{1 \leq j, k \leq m} G_{x}\left(A_{k}\right) \pi_{j}(y)
$$

C'est "la bonne" majoration puisque par le principe du maximum l'inégalité opposée (avec une autre constante $C$ ) a également lieu, y compris si $d=2$.

(ii) Dans le cas $d=2, G_{A_{j}}$ n'est pas uniformément bornée (indépendamment de $R)$ sur $\partial B\left(A_{j}, c_{0} \frac{R}{8}\right)$ en général et (3.1) est peu précise (si on ne fait pas par exemple d'hypothèse sur l'épaisseur en capacité de $B(a, R) \backslash \Omega)$. Néanmoins on trouve la "bonne" estimation en reprenant plus soigneusement la preuve ci-dessus. Observons par exemple que si $\Omega \cap B\left(a, \frac{R}{8}\right) \neq \emptyset, \Omega$ contient une boule $B(b, \alpha R)$ avec $|b-a|=\frac{1}{2}(1 / 4+$ $1 / 8) R=\frac{3}{16} R, \alpha=\frac{1}{16} \min \left\{c_{0}, 1\right\}$ (utiliser un $c_{0}$-arc de John $z \widehat{A}_{j}$ de $\Omega$, $\left.z \in \Omega \cap B\left(a, \frac{R}{8}\right)\right)$ et $V=\Omega \backslash \bar{B}\left(a, \frac{R}{4}\right)$ est contenu dans $\Omega^{\prime}:=\Omega \backslash$ $\bar{B}\left(b, \frac{\alpha}{2} R\right) \subset \Omega$. De plus (exercice facile) $\Omega^{\prime},\left\{A_{j}\right\}_{1 \leq j \leq m}$ et $R$ vérifient les hypothèses du Théorème 3.1 sur $\Omega,\left\{A_{j}\right\}_{1 \leq j \leq m}$ et $R$-avec un nouveau $c_{0}$ - En reprenant les inégalités $(*)$, et en notant $G^{\prime}$ la fonction de Green de $\Omega^{\prime}$, on obtient grâce au 2.B et en passant par $G^{\prime}$,

$$
\text { (3.3) } \mu(y) \leq C \sup _{z \in B\left(a, \frac{3 R}{8}\right)} g_{z}(y) \leq C \sup _{z \in B\left(a, \frac{3 R}{8}\right)} G_{z}^{\prime}(y) \leq C C^{\prime} \sum_{1 \leq j \leq m} G_{A_{j}}^{\prime}(y)
$$

avec $C^{\prime}=C\left(d, c_{0}\right)$.

Comme $\mathbb{R}^{2} \backslash \Omega^{\prime}$ contient $B\left(b, \frac{1}{2} \alpha R\right)$, on a $G_{A_{j}}^{\prime} \leq C^{\prime \prime}$ sur $\partial B\left(A_{j} ; c_{0} R / 8\right)$ (pour une constante absolue $C^{\prime \prime}>0$ ) et $G_{A_{j}}^{\prime} \leq C^{\prime \prime} \pi_{j}$ sur $\Omega \backslash B\left(A_{j} ; c_{0} R / 8\right.$ ) (rappel : $\pi_{j}$ est le potentiel capacitaire de la boule $\bar{B}\left(A_{j}, c_{0} \frac{R}{8}\right)$ dans $\Omega$ ). Finalement, on obtient que pour $x \in \Omega \cap B\left(a ; \frac{R}{8}\right)$ et $y \in \Omega \backslash B\left(a ; \frac{R}{2}\right)$

$$
G_{x}(y) \leq C \sum_{1 \leq j, k \leq m} G_{x}\left(A_{k}\right) \pi_{j}(y)
$$

avec $C=C\left(d, c_{0}\right)>0$. 
Observons maintenant en vue de la suite la variante suivante du Théorème 3.1 .

Corollaire 3.3. Plaçons-nous dans les hypothèses du Théorème 3.1 en supposant de plus que $\delta_{\Omega}\left(A_{k}\right) \geq \frac{c_{0}}{4} R$, pour $1 \leq k \leq m$, et notons $\pi_{k}:=$ $R_{1}^{B_{k}}$ le potentiel capacitaire de la boule $B_{k}:=\bar{B}\left(A_{k}, \frac{c_{0}}{8} R\right)$ dans $\Omega$. Pour chaque $\eta \in\left(0, \frac{1}{2}\right)$ il existe une constante $C=C\left(\eta, c_{0}, d\right)>0$ telle que

$$
G_{x}(y) \leq C \sum_{1 \leq j, k \leq m} G_{x}\left(A_{j}\right) \pi_{k}(y)
$$

pour tout $x \in B\left(a, \frac{\eta}{4} R\right) \cap \Omega$ et tout $y \in \partial B(a, \eta R) \cap \Omega$.

Démonstration: ${ }^{(2)}$ Il existe d'après le Lemme 1.5 des points $A_{k}^{\prime} \in$ $\partial B(a, 2 \eta R) \cap \Omega, 1 \leq k \leq m_{0}=m_{0}\left(c_{0}, d\right)$, tels que chaque point de $B(a, 3 \eta R / 2) \cap \Omega$ (supposé non vide) puisse être joint à un $A_{k}^{\prime}$ par un $\frac{c_{0}}{2}$-arc de John de $\Omega$ et $\delta\left(A_{k}^{\prime}\right) \geq \frac{c_{0} \eta}{2} R$ pour $1 \leq k \leq m$. Par le théorème précédent

$$
G_{x}(y) \leq C R^{d-2} \sum_{1 \leq j, k \leq m_{0}} G_{x}\left(A_{j}^{\prime}\right) G_{A_{k}^{\prime}}(y)
$$

pour $x \in B(a, \eta R / 4) \cap \Omega, y \in \partial B(a, \eta R)$ et une constante $C=C\left(c_{0}, d\right)$. Comme chaque $A_{k}^{\prime}$ peut être joint à un $A_{j}$ par un $c_{0}$-arc de John de $\Omega$ et comme $\delta\left(A_{j}\right) \leq \delta\left(A_{k}^{\prime}\right)+R \leq\left(1+2 c_{0}^{-1} \eta^{-1}\right) \delta\left(A_{k}^{\prime}\right)$ la Proposition 1.2 dit que pour une constante $c^{\prime}=c^{\prime}\left(c_{0}, d, \eta\right)$ on a

$$
G_{x}(y) \leq C c^{\prime} R^{d-2} \sum_{1 \leq j, k \leq m} G_{x}\left(A_{j}\right) G_{A_{k}}(y) .
$$

Ce qui —au moins si $d \geq 3$ - donne le résultat puisque $G_{A_{k}} \leq c \pi_{k}$ sur $\Omega \backslash B\left(A_{k}, \frac{c_{0}}{8} R\right.$ ). Des adaptations faciles (cf. les Remarques 3.2 et la Proposition 1.2bis) étendent (3.5) au cas $d=2$.

\section{Le principe de Harnack au bord dans $\Omega$. Forme forte}

Le but ici est d'établir un principe de Harnack à la frontière plus précis que le précédent, ce qui résoudra le problème soulevé dans $[\mathbf{A H L}$, Remark 2.2]. Il apparait dans [An3] (cf. Définition 2.3) où il est établi pour les domaines de Denjoy lipschitziens. Il est appelé dans [AHL] (cf. Lemma 6.3) "principe de Harnack au bord faible" contrairement à la terminologie adoptée ici. L'idée va être d'étendre la méthode de [An3] (propre au cas $N=2$ et reprise d'ailleurs dans $[\mathbf{A L M}]$ ) à l'aide d'une récurrence inspirée par le travail de Lömker (voir [Löm, p. 37 et suivantes]).

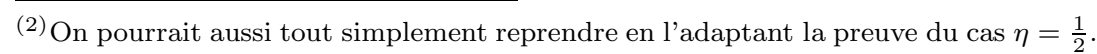


La proposition clef. Considérons un domaine de Green $U$ de $\mathbb{R}^{d}$, un point $a \in \mathbb{R}^{d}$, des points $A_{j} \in \partial B(a, R) \cap U, 1 \leq j \leq N$, et supposons qu'on ait la propriété $P(\Gamma)$ suivante pour une partie $\Gamma$ de $\{1, \ldots, N\}^{2}$ contenant la diagonale $\{(j, j) ; 1 \leq j \leq N\}$ :

"Pour tout $\eta \in\left(0, \frac{1}{2}\right)$, il existe $\varepsilon=\varepsilon(\eta, \Gamma) \in\left(0, \frac{1}{2}\right)$ tel que

$P(\Gamma)$

$$
G^{U}(x, y) \leq c_{\Gamma} \sum_{(j, k) \in \Gamma} G^{U}\left(x, A_{j}\right) G^{U}\left(A_{k}, y\right)
$$

pour tout $x \in U \cap \bar{B}(a, \varepsilon \eta R)$, tout $y \in U \backslash B(a, \eta R)$ et une constante $c_{\Gamma}=c_{\Gamma}(\eta)>0$."

Ici $N$ est un entier $\geq 1$ et on a noté $G^{U}$ la fonction de Green de $U$.

On a alors la propriété clef suivante.

Proposition 4.1. Si $\left(j_{0}, k_{0}\right) \in \Gamma, j_{0} \neq k_{0}$, et si $\Gamma^{\prime}=\Gamma \backslash\left\{\left(j_{0}, k_{0}\right),\left(k_{0}, j_{0}\right)\right\}$, la propriété $P\left(\Gamma^{\prime}\right)$ a également lieu avec $\varepsilon\left(\eta, \Gamma^{\prime}\right)=\varepsilon_{1} \varepsilon_{2}$, où $\varepsilon_{1}=\varepsilon(\eta, \Gamma)$ et $\varepsilon_{2}=\varepsilon\left(\varepsilon_{1} \eta, \Gamma\right)$. On peut d'ailleurs prendre $c_{\Gamma^{\prime}}(\eta)=3\left(c_{\Gamma}\left(\varepsilon_{1} \eta\right)+c_{\Gamma}(\eta)\right)$.

Démonstration: On peut supposer $A_{j_{0}} \neq A_{k_{0}}$. Notons $\varepsilon_{1}=\varepsilon(\eta, \Gamma), \varepsilon_{2}=$ $\varepsilon\left(\varepsilon_{1} \eta, \Gamma\right)$ et définissons $\varepsilon_{0}:=\varepsilon_{1} \varepsilon_{2}=\varepsilon(\eta, \Gamma) \times \varepsilon\left(\varepsilon_{1} \eta, \Gamma\right)$.

Introduisons (comme pour le cas $N=2$, cf. [An3]), les ouverts

$$
\begin{aligned}
U_{j_{0}} & =\left\{z \in U ; G^{U}\left(A_{j_{0}}, z\right)>G^{U}\left(A_{k_{0}}, z\right)\right\} \quad \text { et } \\
U_{k_{0}} & =\left\{z \in U ; G^{U}\left(A_{j_{0}}, z\right)<G^{U}\left(A_{k_{0}}, z\right)\right\} .
\end{aligned}
$$

On peut supposer $x \in \bar{U}_{j_{0}} \cap B\left(a, \varepsilon_{0} \eta\right)$ puisque $U \subset \bar{U}_{j_{0}} \cup \bar{U}_{k_{0}}$ et que le cas $x \in \bar{U}_{k_{0}} \cap B\left(a, \varepsilon_{0} \eta\right)$ est bien sûr semblable au cas où $x \in$ $\bar{U}_{j_{0}} \cap B\left(a, \varepsilon_{0} \eta\right)$.

Soit $F=\partial U_{j_{0}} \cap \bar{B}\left(a, \varepsilon_{1} \eta R\right) \cap U$ et soient $p=\widehat{R}_{G_{x}^{U}}^{F}$ et $g_{x}=G_{x}-p$, où la réduite (ref. $[\mathbf{B r} 4]$ ou $[\mathbf{H e r}]) \widehat{R}_{G_{x}^{U}}^{F}$ est relative au domaine $U$. Notons que $g_{x}$ est la fonction de Green de pôle $x$ dans $V:=U \backslash F$ prolongée par zéro hors de $V$. Si $y \in \partial B\left(a, \varepsilon_{1} \eta R\right) \cap U_{j_{0}}$, alors puisque $y \in U_{j_{0}}$

$$
G^{U}\left(x, A_{j_{0}}\right) G^{U}\left(A_{k_{0}}, y\right) \leq G^{U}\left(x, A_{j_{0}}\right) G^{U}\left(A_{j_{0}}, y\right),
$$

et aussi, puisque $x \in U_{j_{0}}$,

$$
G^{U}\left(x, A_{k_{0}}\right) G^{U}\left(A_{j_{0}}, y\right) \leq G^{U}\left(x, A_{j_{0}}\right) G^{U}\left(A_{j_{0}}, y\right) .
$$


Ainsi, pour $y \in U_{j_{0}} \backslash B\left(a, \varepsilon_{1} \eta R\right)$, on a d'après l'hypothèse (avec $\eta^{\prime}=\varepsilon_{1} \eta$ ) et ce qui précède,

$$
\begin{aligned}
g_{x}(y) \leq G_{x}^{U}(y) & \leq c_{1} \sum_{(j, k) \in \Gamma} G_{x}^{U}\left(A_{j}\right) G^{U}\left(A_{k}, y\right) \\
& \leq 3 c_{1} \sum_{(j, k) \in \Gamma^{\prime}} G_{x}^{U}\left(A_{j}\right) G^{U}\left(A_{k}, y\right)
\end{aligned}
$$

où $c_{1}=c_{\Gamma}\left(\varepsilon_{1} \eta\right)$.

Donc, par le principe du maximum, et puisque le bord dans $V=U \backslash F$ de $U_{k_{0}} \cap V$ est contenu dans $U \backslash B\left(a, \varepsilon_{1} \eta R\right)$, l'inégalité (4.1) entraîne que pour tout $y \in U_{k_{0}}$,

$$
g_{x}(y) \leq 3 c_{1} \sum_{(j, k) \in \Gamma^{\prime}} G_{x}^{U}\left(A_{j}\right) G^{U}\left(A_{k}, y\right) .
$$

D'un autre côté, si $z \in F$ et si $y \in U \backslash B(a, \eta R)$, en utilisant à nouveau l'hypothèse (cette fois pour $\eta$ ) on a,

$$
\begin{aligned}
G_{z}^{U}(y) & \leq c_{2} \sum_{(j, k) \in \Gamma} G^{U}\left(z, A_{j}\right) G^{U}\left(A_{k}, y\right) \\
& \leq 2 c_{2} \sum_{(j, k) \in \Gamma^{\prime}} G^{U}\left(z, A_{j}\right) G^{U}\left(A_{k}, y\right),
\end{aligned}
$$

puisque $G^{U}\left(z, A_{j_{0}}\right)=G^{U}\left(z, A_{k_{0}}\right)$ et donc

$$
G^{U}\left(z, A_{k_{0}}\right) G^{U}\left(A_{j_{0}}, y\right)=G^{U}\left(z, A_{j_{0}}\right) G^{U}\left(A_{j_{0}}, y\right) ;
$$

noter aussi que

$$
G^{U}\left(z, A_{j_{0}}\right) G^{U}\left(A_{k_{0}}, y\right)=G^{U}\left(z, A_{k_{0}}\right) G^{U}\left(A_{k_{0}}, y\right) .
$$

Ici $c_{2}=c_{\Gamma}(\eta)$.

En intégrant par rapport à $z$ l'inégalité (4.3) contre la mesure $-\Delta p$ associée à $p$ dans $U$, on obtient en observant que $p \leq G_{x}^{U}$,

$$
\begin{aligned}
p(y) & \leq 2 c_{2} \sum_{(j, k) \in \Gamma^{\prime}} p\left(A_{j}\right) G^{U}\left(A_{k}, y\right) \\
& \leq 2 c_{2} \sum_{(j, k) \in \Gamma^{\prime}} G^{U}\left(x, A_{j}\right) G^{U}\left(A_{k}, y\right),
\end{aligned}
$$

pour $y \in U \backslash B(a, \eta R)$. Finalement en ajoutant (4.4) et (4.2) (qui a lieu pour tout $y \in U \backslash B(a, \eta R))$,

$$
G^{U}(x, y) \leq 3\left(c_{2}+c_{1}\right) \sum_{(j, k) \in \Gamma^{\prime}} G^{U}\left(x, A_{j}\right) G^{U}\left(A_{k}, y\right),
$$


pour tout $x \in U \cap B\left(a, \varepsilon_{0} \eta R\right)$ et tout $y \in U \backslash B(a, \eta R)$. Ce qui achève la preuve de la proposition clef.

Corollaire 4.2. Soient $U$ un ouvert de Green de $\mathbb{R}^{d}, a \in \mathbb{R}^{d}, R>0$ et $\left\{A_{j}\right\}_{1 \leq j \leq N}$ vérifiant l'hypothèse $P\left(\Gamma_{0}\right)$ avec $\Gamma_{0}=\{1, \ldots, N\}^{2}, c_{\Gamma_{0}}(\eta)=$ $c_{\Gamma_{0}}^{\prime} R^{d-2}$ et $\varepsilon(\eta)=\frac{1}{4}$. Il existe $\varepsilon_{0}=\varepsilon_{0}(N, d), 0<\varepsilon_{0} \leq \frac{1}{4}$ et $C=$ $C\left(c_{\Gamma_{0}}^{\prime}, N\right)$ tels que

$$
G^{U}(x, y) \leq C R^{d-2} \sum_{j=1}^{N} G^{U}\left(x, A_{j}\right) G^{U}\left(A_{j}, y\right)
$$

pour $x \in \partial B\left(a, \varepsilon_{0} R\right) \cap U$ et $y \in \partial B\left(a, \frac{R}{2}\right) \cap U$.

En combinant avec le Théorème 3.1, on obtient une première forme du principe de Harnack au bord "fort" sous les hypothèses du Théorème 3.1.

Corollaire 4.3. Soient $\Omega$ un ouvert de Green de $\mathbb{R}^{d}, R$ un réel $>0$, et soient $a \in \mathbb{R}^{d}$ et $A_{1}, \ldots, A_{m}$ des points de $\partial B(a, R) \cap \Omega$ tels que chaque point $z \in B\left(a, \frac{3}{4} R\right) \cap \Omega$ puisse être joint à au moins un des $A_{j}$ par un $c_{0}$-arc de John de $\Omega$ issu de $z$. On a alors

$$
G_{x}(y) \leq C R^{d-2} \sum_{1 \leq j \leq m} G_{x}\left(A_{j}\right) G_{A_{j}}(y)
$$

pour tout $x \in \partial B\left(a, \frac{R}{4}\right) \cap \Omega$ et tout $y \in \partial B\left(a, \frac{R}{2}\right) \cap \Omega$. Ici $C=C\left(d, c_{0}\right)>0$.

Preuve: D'abord, quitte à réduire l'ensemble des $A_{j}$ et à remplacer $c_{0}$ par $c_{0} / 2$, on peut supposer que $d\left(A_{j}, A_{k}\right) \geq c_{0} \frac{R}{4}$ et donc $m \leq m_{0}\left(c_{0}, d\right)$. Une application directe du Corollaire 4.2 et du Théorème 3.1 donne ensuite que

$$
G_{x}(y) \leq C_{1} R^{d-2} \sum_{1 \leq j \leq m} G_{x}\left(A_{j}\right) G_{A_{j}}(y)
$$

pour $x \in \partial B(a, \varepsilon R) \cap \Omega, 0<\varepsilon=\varepsilon\left(d, m_{0}\right)<\frac{1}{4}, y \in \partial B\left(a, \frac{1}{2} R\right) \cap \Omega$ et une constante $C_{1}=C_{1}\left(d, c_{0}, m_{0}\right)>0$.

En particulier, si $x \in \partial B\left(a, \frac{R}{4}\right) \cap \Omega$ est tel que $\delta(x)<\varepsilon \frac{R}{4}$, si $a^{\prime} \in$ $\partial \Omega$ est tel que $\delta(x)=\delta\left(a^{\prime}\right)$ et si $\left\{A_{j}^{\prime}\right\}_{1 \leq j \leq m_{0}}$ est un système de points de $\partial B\left(a^{\prime}, \frac{R}{4}\right)$ dominant pour $B\left(a^{\prime}, 3 R / 16\right) \cap \Omega$ (cf. le Lemme 1.5) on a pour tout $y \in \partial B\left(a, \frac{R}{2}\right) \subset \Omega \backslash B\left(a^{\prime}, \frac{R}{8}\right)$,

$$
G_{x}(y) \leq C_{1} R^{d-2} \sum_{1 \leq j \leq m_{0}} G_{x}\left(A_{j}^{\prime}\right) G_{A_{j}^{\prime}}(y)
$$

en appliquant (4.7) à $U$, le point $a^{\prime} \in \partial U, R^{\prime}=R / 4$ et les $A_{j}^{\prime}$. En utilisant ensuite (comme dans la preuve du Théorème 2.1) la Proposition 1.2 et 
des arcs de John joignant chaque $A_{j}^{\prime}$ à un $A_{k}$, on a $G_{x}\left(A_{j}^{\prime}\right) \leq c G_{x}\left(A_{k_{j}}\right)$, $G\left(A_{j}^{\prime}, y\right) \leq c G\left(A_{k_{j}}, y\right)$ pour des $k_{j}$ convenables et une constante $c=$ $c\left(d, c_{0}, \varepsilon\right)$. On en déduit l'inégalité voulue :

$$
G_{x}(y) \leq c^{2} C_{1} R^{d-2} \sum_{1 \leq j \leq m} G_{x}\left(A_{j}\right) G_{A_{j}}(y), \quad c=c\left(d, c_{0}, \varepsilon\right) .
$$

Si $x \in \partial B\left(a, \frac{R}{4}\right) \cap \Omega$ est tel que $\delta(x) \geq \varepsilon \frac{R}{4}$, les inégalités de Harnack de la Proposition 1.2 et l'existence d'un arc $c_{0}$-John joignant $x$ à l'un des $A_{j}$ donnent directement $G_{x}(y) \leq C_{2} G_{A_{j}}(y), C_{2}=C_{2}\left(d, c_{0}, \varepsilon\right)$, et aussi $R^{d-2} G\left(x, A_{j}\right) \geq C_{3}, C_{3}=C_{3}\left(d, c_{0}, \varepsilon\right)$ pour au moins un $j, 1 \leq j \leq m$. Donc

$$
G_{x}(y) \leq C_{2} C_{3} R^{d-2} \sum_{1 \leq j \leq m} G_{x}\left(A_{j}\right) G_{A_{j}}(y) .
$$

L'énoncé suivant est équivalent au précédent pour $d \geq 3$ mais il est plus précis pour $d=2$ (voir la fin de la Section 3).

Théorème 4.4. Soit $\Omega$ un ouvert de Green de $\mathbb{R}^{d}$, soient $A_{1}, \ldots, A_{m}$ des points de $\partial B(a, R) \cap \Omega, a \in \mathbb{R}^{d}, R>0$, tels que chaque point $z \in$ $B\left(a, \frac{3}{4} R\right) \cap \Omega$ puisse être joint à au moins l'un des $A_{j}$ par un $c_{0}$-arc de John de $\Omega$ issu de $z$. On a alors

$$
G_{x}(y) \leq C \sum_{1 \leq j \leq m} G_{x}\left(A_{j}\right) \pi_{j}(y)
$$

pour tout $x \in \partial B\left(a, \frac{R}{4}\right) \cap \Omega$ et tout $y \in \partial B\left(a, \frac{1}{2} R\right) \cap \Omega$. Ici $\pi_{j}:=R_{\mathbf{1}}^{B_{j}}$ désigne le potentiel d'équilibre de $B_{j}=B\left(A_{j}, c_{0} \frac{R}{8}\right)$ dans $\Omega$ (i.e. la mesure harmonique de $\partial B_{j}$ dans $\left.\Omega \backslash \bar{B}_{j}\right)$ et $C=C\left(d, c_{0}\right)>0$.

Remarque. Comme avant (cf. le 1.B), quitte à jeter des $A_{j}$ inutiles, on peut supposer en outre $\delta\left(A_{j}\right) \geq c_{0} \frac{R}{4}$. On peut aussi en supprimant encore certains $A_{k}$ supposer que $d\left(A_{j}, A_{k}\right) \geq c_{0} \frac{R}{4}$ pour $1 \leq j \neq k \leq m$-quitte à remplacer $c_{0}$ par $\frac{c_{0}}{2}$.

Preuve: Supposant $d=2$, on adapte facilement la preuve précédente, en utilisant le Lemme 4.5 suivant qui assure qu'il existe $\tilde{C}=\tilde{C}\left(d, c_{0}\right)$ tel que si $Z_{j} \in \partial B\left(A_{j}, c_{0} \frac{R}{8}\right)$ pour $1 \leq j \leq m$, on a $G_{A_{k}}\left(Z_{k}\right) \leq \tilde{C} G_{A_{\ell}}\left(Z_{\ell}\right)$ pour $1 \leq k, \ell \leq m, \tilde{C}=\tilde{C}\left(d, c_{0}\right)$.

On voit alors que le $(3.5)$ du Corollaire 3.3 peut se réécrire sous la forme

$$
G_{x}(y) \leq C \theta^{-1} \sum_{j, k} G_{x}\left(A_{j}\right) G\left(A_{k}, y\right)
$$


avec $\theta=\max \left\{G\left(A_{j}, Z_{j}\right) ; 1 \leq j \leq m, d\left(Z_{j}, A_{j}\right)=\frac{c_{0} R}{8}\right\} \leq \tilde{C}^{-1} \min \left\{G\left(A_{j}, Z_{j}\right) ;\right.$ $\left.1 \leq j \leq m, d\left(Z_{j}, A_{j}\right)=\frac{c_{0} R}{8}\right\}, C=C\left(d, c_{0}\right), \tilde{C}=\tilde{C}(d)$. Le Corollaire 4.2 avec le raisonnement de la proposition précédente donne maintenant

$$
G_{x}(y) \leq C \theta^{-1} \sum_{1 \leq j \leq m} G\left(x, A_{j}\right) G\left(A_{j}, y\right)
$$

pour $x \in \Omega \cap B\left(a, \frac{R}{4}\right), y \in \Omega \backslash B\left(a, \frac{R}{2}\right)$ avec une nouvelle constante $C=$ $C\left(d, c_{0}\right)$ (on utilise d'ailleurs encore le Lemme 4.5). On achève en notant que par le principe du maximum $G_{A_{j}}(y) \leq \theta \pi_{j}(y)$.

Lemme 4.5. Soient $U$ un ouvert de Green de $\mathbb{R}^{d}$ et $B(a, \rho), B(b, \rho)$ deux boules contenues dans $U$ et telles que $d(a, b) \leq c \rho$. Alors on a pour $a^{\prime} \in \partial B\left(a, \frac{\rho}{2}\right), b^{\prime} \in \partial B\left(b, \frac{\rho}{2}\right)$,

$$
C^{-1} G\left(b, b^{\prime}\right) \leq G\left(a, a^{\prime}\right) \leq C G\left(b, b^{\prime}\right)
$$

où $C=C(d, c)$ et où $G$ désigne la fonction de Green de $U$.

Preuve: Pour $d \geq 3$, il est bien classique que $c_{d}\left(1-2^{2-d}\right) \rho^{2-d} \leq G\left(a, a^{\prime}\right) \leq$ $c_{d} \rho^{2-d}$ où $c_{d}$ ne dépend que de $d$. On peut donc supposer $d=2$, cas où on a de même la minoration uniforme $G\left(a, a^{\prime}\right) \geq c_{2} \log (2)=c_{2}^{\prime}$ mais où $G\left(a, a^{\prime}\right)$ n'est pas uniformément borné indépendamment de $U$. Comme par les inégalités de Harnack on peut choisir arbitrairement $b^{\prime} \in \partial B\left(b, \frac{\rho}{2}\right)$ et $a^{\prime} \in \partial B\left(a, \frac{\rho}{2}\right)$, on peut aussi supposer que $a, a^{\prime} \notin B\left(b^{\prime}, \frac{\rho}{2}\right)$.

Dans $U_{a}=U \backslash \bar{B}\left(b^{\prime}, \frac{\rho}{8}\right)$, on a $G_{a}=g_{a}+H$ où $g_{a}$ est la fonction de Green de pôle $a$ dans $U_{a}$ et où $H$ est la solution du problème de Dirichlet dans $U_{a}$ pour la donnée frontière $f=G_{a}$ sur $\partial B\left(b^{\prime}, \frac{\rho}{8}\right)$ et $f=0$ sur $\partial U_{a} \cap \partial U$. On a $g_{a} \leq c^{\prime}$ sur $\partial B\left(a, \frac{\rho}{2}\right)$ - on peut se ramener à $U_{a}=\mathbb{R}^{2} \backslash \bar{B}\left(b^{\prime}, \frac{\rho}{8}\right)$ et à $\rho=1$ - avec $c^{\prime}=c^{\prime}(c)>0$, et aussi $G\left(a, b^{\prime}\right) \leq$ $c^{\prime} G\left(b, b^{\prime}\right)$ (inégalités de Harnack et principe du maximum). Donc

$$
G_{a}\left(a^{\prime}\right) \leq c^{\prime}\left(1+G\left(a, b^{\prime}\right)\right) \leq c^{\prime}\left(1+c^{\prime} G\left(b^{\prime}, b\right)\right) \leq c^{\prime}\left(c_{2}^{-1}+c^{\prime}\right) G\left(b, b^{\prime}\right) .
$$

\section{Autres formes du PHF. Premières applications}

5.A. On va d'abord énoncer d'autres formes, plus naturelles et éventuellement plus commodes, du Théorème 4.4. Commençons par l'énoncé suivant où pour $F \subset \mathbb{R}^{d}$ on note $F_{\eta}:=\left\{x \in \mathbb{R}^{d} ; d(x, F) \leq \eta\right\}$.

Théorème 5.1. Soient $\Omega$ un ouvert de $\mathbb{R}^{d}, F$ un fermé de $\Omega, \eta, c_{0}$, $c_{1}$ des réels $>0, c_{0} \in\left(0, \frac{1}{2}\right), c_{1}>1$ et $A_{j}, 1 \leq j \leq m$, des points de $\Omega$ tels que : (i) $c_{1}^{-1} \eta \leq \delta\left(A_{j}\right) \leq c_{1} \eta$ pour $1 \leq j \leq m$ et (ii) chaque point de $F_{\eta} \cap \Omega$ peut être joint à au moins un des $A_{j}$ par un $c_{0}$-arc de John de $\Omega$. Notons $\pi_{j}:=R_{1}^{B_{j}}$ le potentiel d'équilibre dans $\Omega$ de $B_{j}:=$ $\bar{B}\left(A_{j}, \frac{c_{1}^{-1}}{4} \eta\right) \subset \Omega$. 
Alors pour toute fonction $h$ harmonique positive sur $\Omega$ qui s'annule $\operatorname{sur} \bar{F}_{\eta} \cap \partial \Omega$ (au sens intrinsèque relativement à $\Omega$ cf. le 1.C) on a

$$
h(x) \leq C \sum_{j=1}^{m} h\left(A_{j}\right) \pi_{j}(x)
$$

pour $x \in F$, avec une constante $C=C\left(d, c_{0}, c_{1}\right)>0$ indépendante de $\eta$. Donc, pour toute suite finie $\left\{h_{j}\right\}_{1 \leq j \leq m}$ de fonctions harmoniques positives sur $\Omega$ et tout $x \in F$, on a

$$
h(x) \leq C^{\prime} \sum_{j=1}^{N} \frac{h\left(A_{j}\right)}{h_{j}\left(A_{j}\right)} h_{j}(x), \quad C^{\prime}=C^{\prime}\left(d, c_{0}, c_{1}\right) .
$$

Remarque 5.2. Notons que les inégalités "opposées" $h(x) \geq C^{-1} h\left(A_{j}\right) \pi_{j}(x)$, pour $x \in \Omega$ et une nouvelle constante $C$, sont immédiates par Harnack et le principe du maximum.

Preuve: On peut supposer $\Omega$ Greenien ( $\operatorname{sinon} h$, les $\pi_{j}$ et les $h_{j}$ sont constantes) connexe et $F \neq \emptyset$. On notera $G$ la fonction de Green de $\Omega$.

(a) Montrons d'abord que pour $x \in \partial F_{\eta / 2} \cap \Omega$, on a pour tout $y \in F$,

$$
G_{x}(y) \leq C \sum_{j=1}^{m} G_{x}\left(A_{j}\right) \pi_{j}(y) .
$$

Posons $\rho=\frac{c_{1}^{-1}}{4} \eta$ et observons que d'après les hypothèses et le Lemme 1.5 on peut trouver des $A_{j}^{\prime} \in \partial B\left(x, \frac{\rho}{10}\right) \cap \Omega, 1 \leq j \leq m_{0}=m_{0}\left(c_{0}, d\right)$ tels que chaque point de $B\left(x, \frac{3 \rho}{40}\right) \cap \Omega$ puisse être joint à l'un des $A_{j}^{\prime}$ par un $\frac{c_{0}}{2}$-arc de John de $\Omega$ (d'après le Lemme 1.5). Par le Théorème 4.4 on a donc pour $y \in \Omega \cap \partial B\left(x, \frac{\rho}{20}\right)$,

$$
G_{x}(y) \leq C \sum_{1 \leq j \leq m_{0}} G_{x}\left(A_{j}^{\prime}\right) \pi_{j}^{\prime}(y), \quad C=C\left(d, c_{0}\right)
$$

où $\pi_{j}^{\prime}$ désigne le potentiel capacitaire de $B_{j}^{\prime}=\bar{B}\left(A_{j}^{\prime}, \frac{c_{0} \rho}{80}\right) \subset \Omega$ dans $\Omega$. À l'aide des inégalités de Harnack de la Proposition 1.2 et comme dans la démonstration du Corollaire 4.3 et du Théorème 4.4 (cf. aussi le 2.A), on voit qu'on peut remplacer les $A_{j}^{\prime}$ et les $\pi_{j}^{\prime}, 1 \leq j \leq m_{0}$, par les $A_{k}$ et les $\pi_{k}, 1 \leq k \leq N$, quitte a multiplier la constante $C$ par une nouvelle constante (dépendant de $c_{1}, c_{0}$ et $d$ ). D'où le (5.2).

(b) Prenons alors la fonction harmonique positive $h$ de l'énoncé et considérons sa réduite (relativement à $\Omega$ ) $p=\widehat{R}_{h}^{F^{\prime}}$ où $F^{\prime}=F_{\eta / 2}$. Cette réduite $p$ est un potentiel de Green dans $\Omega$ [cf. le 1.8] de mesure associée 
(dans $\Omega$ ) $\mu:=-\Delta p$ portée par $\partial F^{\prime} \cap \Omega$ et on a $p=h$ sur $F$. Il découle alors des inégalités (5.2) (en intégrant en $x$ contre $\mu$ ) que

$$
h(y) \leq C \sum_{j=1} h\left(A_{j}\right) \pi_{j}(y),
$$

pour tout $y \in F$. Mais par les inégalités de Harnack ordinaires, on a $\pi_{j} \leq c h_{j} / h\left(A_{j}\right)$ sur $B\left(A_{j}, \rho\right)$. Donc par le principe du maximum $\left(\pi_{j}\right.$ est un potentiel sur $\Omega), \pi_{j} \leq c h_{j} / h\left(A_{j}\right)$ sur $\Omega \backslash B\left(A_{j}, \rho\right)$, et en combinant avec (5.4) on obtient l'inégalité (5.1') de l'énoncé.

On peut donner une version un peu plus fine du Théorème 5.1, basée sur la distance accessible dans l'ouvert $\Omega$ de $\mathbb{R}^{d}$. Cette distance $d_{a}=$ $d_{a}^{\Omega}$ est définie par la formule

$$
d_{a}^{\Omega}(x, y)=\inf \{\operatorname{diam}(C) ; C \text { partie connexe de } \Omega, x, y \in C\}
$$

pour $x, y \in \Omega$ (ref. [Br1], [BV1], [ALM] pour des utilisations en théorie du potentiel). On considère maintenant, pour $F \subset \Omega$, les ensembles ouverts $F_{\eta}^{\Omega}=\left\{x \in \Omega ; d_{a}^{\Omega}(x, F)<\eta\right\}, \eta>0$.

Théorème 5.3. Soient $F$ un fermé de $\Omega, \eta$ un réel $>0, c_{0} \in\left(0, \frac{1}{2}\right)$, $c_{1} \geq 1$ et $A_{j}, 1 \leq j \leq m$, des points de $\Omega$ tels que : (i) $c_{1}^{-1} \eta \leq \delta\left(A_{j}\right) \leq$ $c_{1} \eta$ pour $1 \leq j \leq m$ et (ii) chaque point de $F_{\eta}^{\Omega}$ peut être joint $\grave{a}$ un des $A_{j}$ par un $c_{0}$-arc de John de $\Omega$. Si h est harmonique positive sur $\Omega$ telle que $h_{\mid F_{\eta}^{\Omega}}$ soit nulle sur $\overline{F_{\eta}^{\Omega}} \cap \partial \Omega$, si $\pi_{j}$ désigne le potentiel capacitaire de $\bar{B}\left(A_{j}, \eta / 4 c_{1}\right)$ dans $\Omega$ alors pour tout $x \in F$

$$
h(x) \leq C \sum_{j=1}^{m} h\left(A_{j}\right) \pi_{j}(x)
$$

avec une constante $C=C\left(d, c_{0}, c_{1}\right)>0$ indépendante de $\eta$.

Comme ci-dessus, on a facilement l'estimation opposée :

$h(x) \geq C^{-1} \sum_{j=1}^{m} h\left(A_{j}\right) \pi_{j}(x)$ sur $\Omega$ pour tout $x \in \Omega$ et $C=C\left(d, c_{0}, c_{1}\right)$ assez grand.

Preuve: On va se ramener au résultat précédent. Considérons $x \in F$, la boule $B(x, R)$ avec $R=\frac{\eta}{100}$ et la composante connexe $V$ de $B(x, R) \cap \Omega$ contenant $x$. Par construction on a $V \subset F_{2 R}^{\Omega}$.

Choisissons des points $A_{j}^{\prime} \in V \cap \partial B(x, R / 2), 1 \leq j \leq m=m\left(c_{0}, d\right)$, tels que tout point de $V \cap B(x, R / 4)$ puisse être joint à l'un des $A_{j}^{\prime}$ par un $\frac{c_{0}}{2}$-arc de John dans $V$ (voir les Lemmes 1.4 et 1.5). On peut aussi supposer $d\left(A_{j}^{\prime}, \partial V\right) \geq c_{0} R / 8$. Par le théorème précédent [appliqué 
à l'ouvert $V$, au fermé $\bar{B}\left(x, \frac{R}{2}\right) \cap V$ de $V$, aux points $A_{j}^{\prime}$ et au réel $\eta=\frac{R}{4}>$ $0]$ on a $h(x) \leq C \sum_{j=1}^{m} h\left(A_{j}^{\prime}\right) \pi_{j}^{\prime}(x)$ où $\pi_{j}^{\prime}$ désigne le potentiel capacitaire de $B\left(A_{j}^{\prime}, c_{0} \frac{R}{8}\right)$. Les inégalités de Harnack de la Proposition 1.2 le long d'un arc de John joignant chaque $A_{j}^{\prime}$ à un $A_{k}$ donnent alors le résultat voulu.

Remarques 5.4. a) L'énoncé ci-dessus admet une version (qui se déduit de (5.2)) utilisant la fonction de Green : sous les hypothèses du théorème précédent sur $\Omega, F$ et les $A_{j}$, et si $G$ est la fonction de Green de $\Omega$, on a pour tout $x \in \Omega \backslash F_{\eta}^{\Omega}$

$$
\begin{aligned}
& G_{x}(y) \leq C \sum_{j} G_{x}\left(A_{j}\right) \pi_{j}(y) \leq C \sum_{j} \rho_{j} G_{x}\left(A_{j}\right) G_{A_{j}}(y), \quad \forall y \in F, \\
& \quad \text { où } \rho_{j}^{-1}=\max \left\{G_{A_{j}}(z) ; 1 \leq j \leq m, d\left(A_{j}, z\right)=\frac{\eta}{4 c_{1}}\right\} .
\end{aligned}
$$

b) On peut remplacer à droite $\rho_{j}$ par $\eta^{d-2}$ quitte à éventuellement perdre, dans le cas $d=2$, un ordre de grandeur.

5.B. Passons maintenant, en suivant des méthodes bien connues (ref. [An3]), à des applications du Théorème 4.4 (et des variantes précédentes) à la frontière de Martin ([Mar], [Naï], [HW1], [HW2]). On va d'abord énoncer un résultat général, dans l'esprit de [An3].

Soient $\Omega$ un domaine et $x_{0}$ un point de référence fixé de $\Omega$. Pour $F$ partie fermée de $\Omega$ telle que $x_{0} \notin F$, notons $b_{+}(F)$ la réunion des composantes de $\Omega \backslash F$ ne contenant pas $x_{0}$, et $b_{-}(F)$ la composante de $\Omega \backslash F$ contenant $x_{0}$. Disons ici qu'un filtre $\mathcal{F}$ sur $\Omega$ est un bout de $\Omega$ s'il existe une suite $\left\{B_{\nu}\right\}_{\nu \geq 1}$ de parties fermées de $\Omega, x_{0} \notin B_{\nu}$, telle qu'on ait, posant $\omega_{\nu}:=b_{+}\left(B_{\nu}\right):$ (i) $B_{\mu} \subset \omega_{\nu}$ et donc $\omega_{\mu} \subset \omega_{\nu}$ si $\mu>\nu$, (ii) pour tout compact $K$ de $\Omega$ il existe $\nu \geq 1$ tel que $K \subset b_{-}\left(B_{\nu}\right)$, (iii) $\left\{\omega_{\nu}\right\}_{\nu \geq 1}$ est une base du filtre $\mathcal{F}$. Ces définitions sont empruntées à $[$ An3].

On dira que $\left\{B_{\nu}\right\}_{\nu \geq 1}$ est un accès du bout $\mathcal{F}$. On notera aussi $\mathcal{H}_{\mathcal{F}}^{+}:=$ $\left\{u \in \mathcal{H}_{+}(\Omega) ; \forall \nu \geq 1, h_{\mid U_{\nu}}\right.$ est nulle sur $\left.\partial U_{\nu} \cap \partial \Omega\right\}$ où $U_{\nu}:=b_{-}\left(B_{\nu}\right)$ et $\mathcal{H}_{+}(\Omega):=\left\{h: \Omega \rightarrow \mathbb{R}_{+} ; h\right.$ harmonique dans $\left.\Omega\right\}$.

Définition 5.5. Soient $\mathcal{F}$ un bout de $\Omega, N$ un entier $\geq 1$ et $c_{0}>0$. Disons que $\mathcal{F}$ est $c_{0}$-John d'ordre $N$ s'il existe un accès $\left\{B_{\nu}\right\}$ de $\mathcal{F}$ avec pour chaque $\nu \geq 1$ les propriétés suivantes : (i) il existe $N$ points $A_{\nu}^{j} \in \Omega$, $1 \leq j \leq N$ et $\varepsilon_{\nu}>0$ tels que tout point de $\left[B_{\nu}\right]_{\varepsilon_{\nu}}^{\Omega}$ peut être joint à l'un des $A_{\nu}^{j}$ par un $c_{0}$-arc de John de $\Omega$, (ii) $c_{0} \varepsilon_{\nu} \leq \delta\left(A_{\nu}^{j}\right) \leq c_{0}^{-1} \varepsilon_{\nu}$, et (iii) $\left[B_{\nu+1}\right]_{\varepsilon_{\nu+1}}^{\Omega} \cap\left[B_{\nu}\right]_{\varepsilon_{\nu}}^{\Omega}=\emptyset\left(\right.$ donc $\left.B_{\mu} \cap\left[B_{\nu}\right]_{\varepsilon_{\nu}}^{\Omega}=\emptyset, \forall \mu>\nu\right)$. 
Théorème 5.6. Soient $\Omega \subset \mathbb{R}^{d}$ un domaine de Green et $\mathcal{F}$ un bout de $\Omega$ qui est c $c_{0}$-John d'ordre $N$. Alors le cône $\mathcal{H}_{\mathcal{F}}^{+}$est de dimension finie $N_{\mathcal{F}}$, engendré par $N_{\mathcal{F}}$ minimales sur $\Omega$ et $1 \leq N_{\mathcal{F}} \leq N$. De plus, si on note $F:=\left\{\zeta \in \partial \widehat{\Omega} ; K_{\zeta} \in \mathcal{H}_{\mathcal{F}}^{+}\right\}$, on a $\mathcal{F}=\{V \cap \Omega ; V$ voisinage dans $\widehat{\Omega}$ de $F\}$.

Ici et dans la suite on note - selon l'usage - $\widehat{\Omega}$ le compactifié de Martin de $\Omega, \partial \widehat{\Omega}=\widehat{\Omega} \backslash \Omega$ la frontière de Martin $\Omega$ et $K_{x}=\lim _{z \in \Omega, z \rightarrow x} \frac{G(., z)}{G\left(x_{0}, z\right)}$ la fonction harmonique positive (ou noyau Martin) associée à $x \in \partial \widehat{\Omega}$ ( $x_{0}$ étant pris comme point de normalisation).

Preuve (Indications rapides): On reprend assez directement les arguments de [An3, partie 2]. (a) Si $h, h_{1}, \ldots, h_{N}$ sont $N+1$ éléments de $\mathcal{H}_{\mathcal{F}}^{+}$on a par le Théorème 5.3 et le principe du maximum : $h(x) \leq$ $C \sum_{j=1}^{N} \frac{h\left(A_{\nu}^{j}\right)}{h_{j}\left(A_{\nu}^{j}\right)} h_{j}(x)$ pour tout $x \in b_{-}\left(B_{\nu}\right)$ et une constante $C$ indépendante de $\nu$. Une répétition de l'argument de [An3, pp. 223-224] montre alors qu'étant donnés $N+1$ éléments, $\mathcal{H}_{\mathcal{F}}^{+}$, l'un d'entre eux est majoré par $C$ fois la somme des autres et que par conséquent $\mathcal{H}_{\mathcal{F}}^{+}$admet un ensemble générateur ayant au plus $N$ éléments. De plus les inégalités utilisées montrent que $\mathcal{H}_{\mathcal{F}}^{+}$est fermé. Le cône $\mathcal{H}_{\mathcal{F}}^{+}$est donc engendré par ses génératrices extrêmales et vu la définition de $\mathcal{H}_{\mathcal{F}}^{+}$ces génératrices sont aussi extrêmales dans $\mathcal{H}_{+}(\Omega)$. D'où la première assertion de l'énoncé.

(b) Soit $h$ harmonique positive dans $\Omega$, limite d'une suite de potentiels normalisés $h_{k}()=.\frac{G\left(., z_{k}\right)}{G\left(x_{0}, z_{k}\right)}, z_{k} \in \omega_{\nu_{k}}, \nu_{k} \uparrow \infty$. D'abord, on a (toujours en utilisant le Théorème 5.3)

$$
h_{k}(x) \leq C \sum_{j=1}^{N} h_{k}\left(A_{\nu}^{j}\right) G_{A_{\nu}^{j}}(x), \quad x \in b_{-}\left(B_{\nu}\right)
$$

pour $\nu_{k}>\nu$. Faisant tendre $k$ vers $+\infty$ on obtient que

$$
h(x) \leq C \sum_{j=1}^{N} h\left(A_{\nu}^{j}\right) G_{A_{\nu}^{j}}(x), \quad x \in b_{-}\left(B_{\nu}\right),
$$

ce qui montre que $h \in \mathcal{H}_{\mathcal{F}}^{+}$.

(c) Inversement si $h \in \mathcal{H}_{+}(\Omega)$ est limite d'une suite $h_{k}()=.\frac{G\left(., z_{k}\right)}{G\left(x_{0}, z_{k}\right)}$ avec des $z_{k}$ appartenant tous à un même $b_{-}\left(B_{\nu-1}\right)$, on aura

$$
h(x) \leq C \sum_{j=1}^{N} h_{k}\left(A_{\nu}^{j}\right) G_{A_{\nu}^{j}}(x), \quad x \in \omega_{\nu} .
$$


Si $h \in \mathcal{H}_{\mathcal{F}}^{+}$alors $h=0$ sur $\partial \Omega$ (frontière dans $\overline{\mathbb{R}}^{d}$ ) et $h=0$ ce qui est absurde.

En application, considérons d'abord le cas de la frontière de Martin d'un domaine $\Omega$ qui est de John au voisinage d'un point frontière. Voir aussi $[\mathbf{A H L}]$.

Plus précisément, disons que le point frontière $a \in \partial \Omega$ de l'ouvert $\Omega \subset$ $\mathbb{R}^{d}$ est un point frontière de $\Omega$ de type John d'ordre inférieur ou égal à l'entier $N \geq 1$, s'il existe $c_{0} \in\left(0, \frac{1}{2}\right)$, une suite $\left\{R_{p}\right\}_{p \geq 0}$ de réels $>0$ tendant vers 0 , une famille $\left\{A_{j}^{p}\right\}_{p \geq 0,1 \leq j \leq N}$ de points de $\Omega$ tels que (i) $c_{0} R_{p} \leq$ $\delta\left(A_{j}^{p}\right) \leq c_{0}^{-1} R_{p}$, (ii) tout point $x \in \Omega \cap B\left(a, c_{0} R_{p}\right)$ est origine d'un $c_{0}$-arc de John dans $\Omega$ d'extrémité l'un des $A_{j}^{p}, 1 \leq j \leq N$.

D'autre part, pour $F$ partie de la frontière (dans $\overline{\mathbb{R}}^{d}$ ) $\partial \Omega \mathrm{du}$ domaine $\Omega$, notons $\mathcal{H}_{F}^{+}(\Omega)$ le cône des fonctions harmoniques positives sur $\Omega$ qui s'annulent (au sens intrinsèque, cf. le 1.C) sur $\partial \Omega \backslash F$.

Le Théorème 5.6 a alors la conséquence suivante. La preuve sera laissée au lecteur.

Proposition 5.7. Si $\Omega$ est de Green et si a est un point frontière de $\Omega$ de type John d'ordre $\leq N$, alors le cône $\mathcal{H}_{a}^{+}(\Omega)$ est engendré par $N^{\prime}$ minimales, $1 \leq N^{\prime} \leq N$. Le filtre des traces sur $\Omega$ des voisinages de a dans $\mathbb{R}^{d}$ est identique à celui des traces sur $\Omega$ des voisinages dans $\widehat{\Omega}$ de $F_{a}:=\left\{x \in \partial \widehat{\Omega} ; K_{x} \in \mathcal{H}_{a}^{+}(\Omega)\right\}$.

On peut d'ailleurs facilement étendre ce qui précède aux points frontières accessibles de $\Omega$. La frontière accessible $\partial_{a} \Omega$ du domaine $\Omega$ est, par définition, l'espace $\bar{\Omega}^{a} \backslash \Omega$, où $\bar{\Omega}^{a}$ désigne le complété de l'espace métrique $\left(\Omega, d_{a}\right)$. Il est clair que l'identité se prolonge en une projection continue $\pi: \bar{\Omega}^{a} \rightarrow \bar{\Omega}$ telle que $\pi\left(\partial_{a} \Omega\right) \subset \partial \Omega$. On notera $B_{a}(\xi, \rho)$ la boule ouverte de centre $\xi \in \partial_{a} \Omega$ et de rayon $\rho$ dans $\left(\bar{\Omega}^{a}, d_{a}\right)$ et $\mathcal{H}_{\xi}^{+}(\Omega)=\{h \in$ $\mathcal{H}_{+}(\Omega) ; h_{\mid \Omega \backslash \bar{B}_{a}(\xi, r)}=0$ sur $\left.\partial\left(\Omega \backslash \bar{B}_{a}(\xi, r)\right) \cap \partial \Omega, \forall r>0\right\}$ (frontières relatives à $\overline{\mathbb{R}^{d}}$ ).

Disons que le point $\xi \in \partial_{a} \Omega$ est John d'ordre $N$ s'il existe $\beta \in\left(0, \frac{1}{2}\right)$, $c_{0}>0$, une suite $\left\{R_{n}\right\}_{n \geq 1}$ de réels $>0$ tendant vers zéro et pour chaque $n \geq 1$ une suite $\left\{A_{n}^{k}\right\}_{1 \leq k \leq N}$ de $N$ points de $\partial B_{a}\left(\xi, R_{n}\right) \cap \Omega$ qui soit $c_{0}$-dominante pour $B_{a}\left(\xi, \beta R_{n}\right)$ dans $\Omega$.

Proposition 5.7bis. Si $\Omega$ est de Green et si $\xi \in \partial_{a} \Omega$ est de type John d'ordre $\leq N$, alors $\mathcal{H}_{\xi}^{+}(\Omega)$ est engendré par un ensemble de $N^{\prime}$ harmoniques minimales, $1 \leq N^{\prime} \leq N$. Le filtre des traces sur $\Omega$ des voisinages de $\xi$ dans $\bar{\Omega}_{a}$ est identique à celui des des voisinages dans $\widehat{\Omega}$ de $M_{\xi}:=\left\{\zeta \in \partial \widehat{\Omega} ; K_{\zeta} \in \mathcal{H}_{\xi}^{+}(\Omega)\right\}$. 
Mentionnons - en vue de la Partie 8- le théorème de type FatouDoob, facilement disponible ici à l'aide d'arguments standards (cf. [BD] et [An1]). Soit $\xi \in \partial_{a} \Omega$ et soit $\mathbb{T} \subset \Omega$ de la forme $\mathbb{T}=\bigcup_{n \geq 1} \mathbb{T}_{n}$ avec $\mathbb{T}_{n}=\left\{x \in \Omega ; \delta(x) \geq \varepsilon d_{a}(x, \xi), \varepsilon \rho_{n} \leq d_{a}(x, \xi) \leq \beta \rho_{n}\right\}$, où $\left\{\rho_{n}\right\}$ est une suite de réels $>0$ décroissant vers 0 et $0<\varepsilon<\beta$.

Théorème 5.8. Supposons que pour chaque $n \geq 1$ il existe $A_{n} \in$ $\partial B_{a}\left(\xi, \rho_{n}\right) \cap \Omega$ qui soit $c_{0}$-dominant pour $B_{a}\left(\xi, \beta \rho_{n}\right) \cap \Omega$ dans $\Omega\left(c_{0}\right.$, $\beta \in\left(0, \frac{1}{2}\right)$ réels indépendants de $\left.n\right)$. Soit $X$ le point minimal de $\partial \widehat{\Omega}$ associé $\grave{a} \xi$ par la Proposition 5.7bis et soient $u, v$ des fonctions harmoniques positives sur $\Omega$. Si le rapport $\frac{u}{v}$ admet une limite fine $\ell \in \mathbb{R}_{+}$ en $X$, alors $\ell=\lim _{x \in \mathbb{T}, d_{a}(x, X) \rightarrow 0} \frac{u(x)}{v(x)}$.

Indications. On montre d'abord que pour $B=B\left(x, \frac{\varepsilon^{2}}{4} \rho_{n}\right), n \geq 1$, $x \in \mathbb{T}_{n}$, on a $R_{K_{X}}^{B}\left(x_{0}\right) \geq c_{1} K_{X}\left(x_{0}\right)=c_{1}$ avec $c_{1}$ indépendant de $x$ et $n$. Une utilisation de la Proposition 1.2 ramène au cas où $d_{a}(\xi, x)=\varepsilon \rho_{n}$; or, dans ce cas le Théorème 5.3 (avec $N=1, F=\partial B_{a}\left(\xi ; \beta \rho_{n}\right)$ voir aussi le 5.4) donne que $c^{-1} K_{x}\left(A_{n}\right) \leq K_{X}\left(A_{n}\right) \leq c K_{x}\left(A_{n}\right)$. La Proposition 1.2 montre ensuite que $c^{-1} K_{x} \leq K_{X} \leq c K_{x}$ sur $\partial B$ avec une nouvelle constante $c$. Donc, par le principe du maximum, $R_{K_{X}}^{B_{n}} \geq c^{-1} K_{x}$ sur $\Omega \backslash B$ et le résultat voulu.

On en déduit qu'une suite de boules $B_{n}=B\left(x_{n}, \frac{\varepsilon}{4} \delta\left(x_{n}\right)\right), x_{n} \in \mathbb{T}_{n}$, $n \geq 1$, n'est pas de réunion effilée en $X$, puisque l'effilement en $X$ entraîne que $\lim _{n \rightarrow \infty} R_{K_{x}}^{B_{n}}=0$ uniformément sur tout compact de $\Omega$.

Une fois ce dernier point établi, le raisonnement standard de [BD] (ou [An1]) donne le résultat voulu.

\section{C. Cas des domaines de John. Utilisation de la distance géodésique.}

Dans cette partie $\Omega$ est un ouvert de $\mathbb{R}^{d}$, borné et $c_{0}$ John relativement à un système fini $\left\{A_{j}\right\}_{1 \leq j \leq m}$ de pôles (ou centres) $A_{j} \in \Omega, m \geq 1$, $c_{0}>0$. Cela signifie que chaque $x \in \Omega$ est l'origine d'un $c_{0}$-arc de John de $\Omega$ d'extrémité l'un des $A_{j}$ (ref. [AHL]). Si $\Omega$ est connexe, $\Omega$ est alors John de centre tout point de $\Omega$ mais sa constante de John ne peut être controlée à l'aide de $c_{0}, d$, le diamètre de $\Omega$ et les distances $\delta_{\Omega}\left(A_{j}\right)$.

Rappelons que la distance géodésique $d_{g}$ dans $\Omega$ (ref. [Va2]) est obtenue en posant, pour $(x, y) \in \Omega$,

$$
d_{g}(x, y):=\inf \{\operatorname{long}(\gamma) ; \gamma:[0, \ell] \rightarrow \Omega \text { arc rectifiable joignant } x \text { à } y\}
$$


(si $\Omega$ est non connexe $d_{g}$ et $d_{a}$ ne sont pas exactement des distances puisque $d_{g}(x, y)=d_{a}(x, y)=+\infty$ pour $x$ et $y$ dans des composantes distinctes de $\Omega$ ). Évidemment $d_{a} \leq d_{g}$, mais d'après un résultat de Väisälä, il existe $C \geq 1$ tel que $d_{g} \leq C \bar{d}_{a}$ (cf. [Va2, $\S 3.3$ et $\left.\S 3.4\right]$ ). En particulier $\bar{\Omega}_{a}$ coïncide avec le complété géodésique $\bar{\Omega}_{g}$ (le complété de $\left(\Omega, d_{g}\right)$ ) et la frontière accessible $\partial_{a} \Omega$ coïncide avec la frontière géodésique $\partial_{g} \Omega=$ $\bar{\Omega}_{g} \backslash \Omega$.

Notons que le diamètre géodésique de chaque composante de $\Omega$ est borné : chaque $x \in \Omega$ peut être joint à un $A_{j}, j=j_{x}$, par un arc $\gamma$ dans $\Omega$ de longueur inférieure à $C_{1}:=c_{0}^{-1} \delta\left(A_{j}\right)$. Il s'ensuit d'ailleurs que $\gamma$ admet une reparamétrisation $\tilde{\gamma}:[0,1] \rightarrow \bar{\Omega}$ qui est $C_{1}$-lipschitzienne. Plus généralement, tout $c_{0}$-arc de John de $\bar{\Omega}_{g}$ (on convient que $\delta(x)=0$ pour $\left.x \in \partial_{g} \Omega\right)$ d'extrémité $A_{j}$ admet une reparamétrisation $\tilde{\gamma}:[0,1] \rightarrow$ $\bar{\Omega}_{g}$ qui est $C_{1}$ lipschitzienne. On en déduit la compacité de $\bar{\Omega}_{g}$.

Lemme 5.9. Le complété géodésique (ou accessible) $\left(\bar{\Omega}_{g}, d_{g}\right)$ est un espace métrique compact.

Preuve: L'ensemble $\mathcal{K}$ des $c_{0}$-arcs de John $\gamma:[0,1] \rightarrow \bar{\Omega}_{g} C_{1}$-Lipschitz et d'extrémité un des $A_{j}$, est compact pour la topologie de la convergence uniforme sur $[0,1]$. Et l'image de $\mathcal{K}$ par l'application continue $\gamma \mapsto \gamma(0) \in$ $\bar{\Omega}_{g}$ contient $\Omega$ et est compacte dans $\bar{\Omega}_{g}$.

Revenant à la compactification de Martin $\widehat{\Omega}$ de $\Omega$, la Proposition 5.7bis admet le corollaire suivant (comparer [AHL, Theorem 1.1]). On note $\partial_{1} \widehat{\Omega}$ la partie minimale de la frontière de Martin de $\Omega$.

Théorème 5.10. L'identité se prolonge en une surjection continue $\pi: \widehat{\Omega} \rightarrow \bar{\Omega}_{g}$. Si $\xi \in \partial_{g} \Omega=\partial_{a} \Omega$ admet un système de référence de moins de $N$ points, on a $1 \leq \#\left(\pi^{-1}(\xi) \cap \partial_{1} \widehat{\Omega}\right) \leq N$.

Exemple 5.11. Si chaque point $\xi \in \partial_{g} \Omega$ admet un système de référence d'ordre $N=1$, l'application canonique $\pi: \widehat{\Omega} \rightarrow \bar{\Omega}_{g}$ est un homéomorphisme et chaque point de $\partial \widehat{\Omega}$ est minimal.

\section{Domaines intérieurement uniformes et principe de Harnack au bord}

6.A. Rappelons d'abord une définition de l' "uniformité intérieure" (ref. [Va2], [Va3], [BV2], [AHL], [BHK]). 
Définitions 6.1. a) Soit $\gamma:[\alpha, \beta] \rightarrow \Omega$ un arc rectifiable dans l'ouvert $\Omega$ de $\mathbb{R}^{d}$ et soit $c>0$. On dira que $\gamma$ est un arc $c$-uniforme ${ }^{(3)}$ (ou $c$ John bilatère) dans $\Omega$ si

(i) $\delta_{\Omega}(\gamma(t)) \geq c \min \left\{\operatorname{long}\left(\gamma_{\mid[\alpha, t]}\right), \operatorname{long}\left(\gamma_{\mid[t, \beta]}\right)\right\}$ pour $\alpha \leq t \leq \beta$, et si de plus

$$
d_{g}(\gamma(\alpha), \gamma(\beta)) \geq c \operatorname{long}(\gamma) .
$$

b) Le domaine $\Omega$ est intérieurement uniforme s'il existe $c>0$ tel que pour chaque couple $(a, b) \in \Omega^{2}$, il existe un arc $c$-uniforme dans $\Omega$ joignant $a$ à $b$. On dira alors que $\Omega$ est $c$-intérieurement uniforme.

Remarque. Si $\Omega$ est borné et intérieurement uniforme au sens ci-dessus, il est $c_{1}$ John pour une constante $c_{1}=c_{1}(\Omega)>0$ et par [Va2, Theorem 3.4] on a $d_{a} \leq d_{g} \leq C d_{a}$ avec $C=C(\Omega)>0$. Dans la définition ci-dessus de l'uniformité intérieure de $\Omega$ on peut donc remplacer (ii) par : (ii)' $d_{a}(\gamma(\alpha), \gamma(\beta)) \geq c \operatorname{long}(\gamma)$.

6.B. Voici maintenant une caractérisation de l'uniformité en termes de propriétés géométriques analogues à celles de l'énoncé du Théorème 4.4 avec $N=1$. Soit $\Omega$ un domaine de $\mathbb{R}^{d}$; pour $\xi \in \bar{\Omega}_{g}$ et $r>0$, on notera $B_{g}(\xi, r)$ la boule ouverte de centre $\xi$ et de rayon $r$ dans $\left(\bar{\Omega}_{g}, d_{g}\right)$ le complété de $\left(\Omega, d_{g}\right)$.

Proposition 6.2. Supposons $\Omega$ borné et la propriété suivante où $R_{0}$, $c_{0}, \beta$ sont des réels $>0$, et $\beta, c_{0} \in\left(0, \frac{1}{2}\right):$ pour $\xi \in \partial_{g} \Omega$ et $0<R \leq$ $R_{0}$, il existe $A \in\left(\bar{B}_{g}(\xi, R) \backslash B_{g}(\xi, \beta R)\right) \cap \Omega$ tel que chaque point $z \in$ $B_{g}\left(\xi, \beta^{2} R\right) \cap \Omega$ peut être joint à $A$ par un $c_{0}$-arc de John dans $\Omega$. Alors, $\Omega$ est intérieurement uniforme.

Une réciproque est donnée plus loin dans la Proposition 6.4.

Démonstration: a) Soient $x, y \in \Omega$ tels que $d(x, y) \leq d_{g}(x, y) \leq \beta^{2} R_{0} / 10$, $\delta(y) \leq \delta(x)$.

Si $d(x, y) \leq \frac{1}{2} \delta(x)$, le segment $[x, y]$ est un arc 1-uniforme de longueur $d(x, y)=d_{g}(x, y)$ dans $\Omega$.

Si $d(x, y)>\frac{1}{2} \delta(x)$, fixons $\xi \in \partial_{g} \Omega$ tel que $d_{g}(x, \xi)=\delta(x)$, posons $R=$ $\beta^{-2}\left(d_{g}(x, y)+\delta(x)\right) \leq 3 \beta^{-2} d_{g}(x, y) \leq R_{0}$ et considérons $A \in\left(\bar{B}_{g}(\xi, R) \backslash\right.$ $B_{g}(\xi, \beta R) \cap \Omega$ avec la propriété de l'énoncé. On a $x, y \in \bar{B}_{g}\left(\xi, \beta^{2} R\right)$ et il existe des $c_{0}$-arcs de John $\gamma_{1}$ et $\gamma_{2}$ joignant respectivement $x$ et $y$ à $A$ (donc de longueurs majorées par $c_{0}^{-1} R \leq 3 c_{0}^{-1} \beta^{-2} d_{g}(x, y)$ ). L'arc $\Gamma$

\footnotetext{
(3) On devrait dire plus précisément "arc c-intérieurement uniforme".
} 
obtenu par concaténation de $\gamma_{1}$ et $\gamma_{2}^{-1}$ est un arc de John (bilatère) dans $\Omega$ pour la constante $\frac{1}{6} c_{0} \beta^{2}$.

b) Supposons $d_{g}(x, y) \geq \beta^{2} R_{0} / 10$. D'après l'hypothèse sur $\Omega$, il existe des $c_{0}$-arcs de John $\gamma_{1}, \gamma_{2}$ joignant $x$ et $y$ respectivement à des points $X, Y$ de $\Omega$ tels que $\delta(X), \delta(Y) \geq \beta^{\prime} R_{0}, \beta^{\prime}=\min \left\{\beta^{2}, c_{0}\left(\beta-\beta^{2}\right)\right\}$. Soit $F$ une partie maximale de $K:=\left\{z \in \Omega ; \delta(z) \geq \beta^{\prime} R_{0}\right\}$ dont les points soient mutuellement à distance $\geq \frac{1}{2} \beta^{\prime} R_{0}$; en particulier $\# F \leq[\operatorname{diam}(\Omega)]^{d} /\left[\frac{1}{2} \beta^{\prime} R_{0}\right]^{d}$. En prenant $X^{\prime}, Y^{\prime} \in F$ tels que $d\left(X, X^{\prime}\right), d\left(Y, Y^{\prime}\right) \leq \frac{1}{2} \beta^{\prime} R_{0}$, un arc rectifiable - ne dépendant que de $X^{\prime}$ et $Y^{\prime}$ - joignant $X^{\prime}$ et $Y^{\prime}$, et les segments $\left[X, X^{\prime}\right],\left[Y, Y^{\prime}\right]$ on joint $X$ et $Y$ par un arc de longeur majorée par une constante $C_{1}(\Omega)$ et à distance de $\Omega^{c}$ minorée par une autre constante. En ajoutant les $\operatorname{arcs} \gamma_{1}$ et $\gamma_{2}^{-1}$, on obtient un $\operatorname{arc} c$-uniforme de $\Omega$ avec $c=c(\Omega)$.

Lemme 6.3. Soient $P, Q \in \Omega$ et $\beta>0$ tels que $P \in B_{g}(Q, \beta \delta(Q))$. Si $\gamma$ est un arc $C$-uniforme dans $\Omega$ joignant $P$ à $Q, 0<C \leq 1$, alors $\gamma$ est un $c_{0}$-arc de John issu de $P$ dans $\Omega$ pour $c_{0}=\min \left\{C, \frac{1}{2} \beta^{-1} C^{2}\right\}$.

Preuve: Notant $\ell$ la longueur de $\gamma$, on a $\ell \leq C^{-1} d_{g}(P, Q) \leq \beta C^{-1} \delta(Q)$. Soit $X$ un point sur $\gamma$ tel que le sous-arc $\gamma_{X}=\widehat{X Q}$ de $\gamma$ soit de longueur inférieure à $\frac{1}{2} \ell$ (sinon la $C$-condition de John est entraînée par le caractère $C$-uniforme de l'arc). Si la longueur de $\gamma_{X}$ est minorée par $\frac{1}{2} \delta(Q)$, alors $\delta(X) \geq \frac{C}{2} \delta(Q) \geq \frac{1}{2} \beta^{-1} C^{2} \ell$ d'après l'uniformité de $\gamma$. Si au contraire $\operatorname{long}\left(\gamma_{X}\right) \leq \frac{1}{2} \delta(Q)$, on a $d(X, Q) \leq \frac{1}{2} \delta(Q)$ et $\delta(X) \geq$ $\frac{1}{2} \delta(Q) \geq \frac{1}{2} \beta^{-1} C \ell$. Dans les deux cas, on a $\delta(X) \geq \frac{1}{2} \beta^{-1} C^{2}$ long $(\gamma)$.

Proposition 6.4. Soit $\Omega$ un domaine borné et c-intérieurement uniforme dans $\mathbb{R}^{d}$. Si $R_{0}>0$ est assez petit, on peut pour chaque $\beta>0$ trouver un réel $c_{0}=c_{0}(\beta, \Omega)>0$ tel que : si $\xi \in \partial_{g} \Omega$ et $0<R<R_{0}$, il existe $A \in \partial B_{g}(\xi, R) \cap \Omega$ qui est $c_{0}$-dominant pour $B_{g}(\xi, \beta R) \cap \Omega$ dans $\Omega$.

Preuve: Comme $\delta_{\Omega} \leq \frac{1}{2} \operatorname{diam}(\Omega):=\frac{1}{2} \Delta_{0}$ sur $\Omega$, pour $x, y \in \Omega$ on a $\frac{c}{2} d_{g}(x, y) \leq \sup _{z \in \Omega} \delta(z) \leq \frac{1}{2} \Delta_{0}$ et $\Omega$ est de diamètre géodésique inférieur à $c^{-1} \Delta_{0}$. Donc d'après le Lemme 6.3 , si on fixe une boule $B\left(x_{0}, R_{0}\right)$ contenue dans $\Omega$, l'ouvert $\Omega$ est $c$ John de centre $x_{0}$ pour un $c>0$ convenable.

Soient $\xi \in \partial_{g} \Omega, 0<R<R_{0}$, et $A$ le premier point d'intersection d'un $c$-arc de John $\widehat{\xi x_{0}}$ de $\Omega$ avec $\partial B_{g}(\xi, R)$. On a $\delta(A) \geq c R$ et pour $x \in$ $B_{g}(\xi, \beta R)$, on a $x \in B_{g}(A,(1+\beta) R)$. L'uniformité de $\Omega$ et le Lemme 6.3 fournissent un $c$-arc de John joignant $x$ à $A$ avec une constante $c_{0}=$ $c_{0}(\beta, \Omega)>0$. 
En particulier, on voit que le Théorème 5.10 redonne un résultat de $[\mathbf{A L M}]$ (cf. aussi $[\mathbf{A i k}]$ ) : si $\Omega$ est borné et intérieurement uniforme la compactification de Martin $\widehat{\Omega}$ est équivalente à la complétion $\left(\bar{\Omega}, d_{g}\right)$.

Remarque 6.5. Si $\Omega$ est en outre uniformément régulier ce dernier résultat découle de $[\mathbf{A n 3}],[\mathbf{A n 5}]$ et $[\mathbf{B H K}]$. En effet si $g_{h}$ est la métrique pseudo-hyperbolique $g_{h}(d x)=\delta_{\Omega}(x)^{-2}|d x|^{2}, x \in \Omega$, l'espace métrique $\left(\Omega, g_{h}\right)$ est d'après [BHK] complet, géodésique et Gromov-hyperbolique et sa compactification visuelle est équivalente à la complétion géodésique $\bar{\Omega}_{g}$. L'opérateur $L=\delta_{\Omega}^{2} \Delta$ est adapté sur $\left(\Omega, g_{h}\right)$ (au sens de [An3], [An5]) et comme $\Omega$ est uniformément régulier (cf. [An4]) $L+\varepsilon$ admet une sursolution $>0$ finie dans $\Omega$. La théorie de [An5] et l'adaptation [An6, Chapter 5] (cf. aussi $[\mathbf{B H K}]$ ) donnent alors le résultat. Voir aussi $[\mathbf{B V 1} \mathbf{1}$.

6.C. Dans toute la suite de cette Partie $6, \Omega$ désigne un domaine borné et intérieurement uniforme de $\mathbb{R}^{d}$ de constante d'uniformité notée $c_{\Omega}$. On va répertorier des formes du principe de Harnack au bord dans ce cadre, formes associées à la donnée d'un arc de John (bilatère ou unilatère) qui semblent naturelles et utiles (cf. les Sections 8 et 9). Commençons par le corollaire suivant du Théorème 5.3 et de la Proposition 6.4.

Corollaire 6.6. Soient $\xi \in \partial_{g} \Omega, \bar{B}\left(x_{0}, r_{0}\right) \subset \Omega, R>0$ tels que $d_{g}\left(\xi, x_{0}\right)>$ $R+r_{0}$ et soit $A$ le premier point d'intersection d'un $c_{0}$-arc de John (unilatère) $\widehat{\xi x_{0}}$ de $\Omega$ avec $\partial B_{g}(\xi, R)$.

Alors, si $0<\beta^{\prime}<\beta<1$, si $x, y \in \Omega$ et $d_{g}(\xi, x) \leq \beta^{\prime} R, d_{g}(\xi, y) \geq \beta R$, on a

$$
G(x, y) \leq c \delta(A)^{d-2} G(x, A) G(A, y)
$$

pour une constante $c=c\left(\Omega, c_{0}, \beta^{\prime}, \beta\right)>0$.

Preuve: D'après la démonstration de la Proposition 6.4, le point $A$ est $c_{1}$-dominant pour $B_{g}\left(\xi,\left(2 \beta-\beta^{\prime}\right) R\right) \cap \Omega$ dans $\Omega$ avec $c_{1}=c_{1}\left(\Omega, c_{0}, \beta\right)$. Appliquant le Théorème 5.3 (cf. les Remarques 5.4) à $F=\partial B_{g}(\xi, \beta R) \cap \Omega$ et $\eta=\left(\beta-\beta^{\prime}\right) R$ avec $N=1$ et $A_{1}=A, x \in B\left(\xi, \beta^{\prime} R\right)$, on obtient que

$$
G_{x}(z) \leq c \delta(A)^{d-2} G_{x}(A) G_{A}(z), \forall z \in \partial B_{g}(\xi, \beta R)
$$

avec $c=c\left(\Omega, c_{0}, \beta^{\prime}, \beta\right)>0$. D'où l'assertion par le principe du maximum. 
Proposition 6.7 (Principe de Harnack au bord associé à un arc de John). Soit $\Gamma$ un $c_{0}$-arc de John joignant le point $P$ au point $Q$ dans $\Omega^{(4)}$. Supposons que $d(P, Q) \geq c_{0} \delta(P)$. On a alors

$$
G(P, R) \leq c \delta(Q)^{d-2} G(P, Q) G(Q, R)
$$

pour tout $R \in \Omega$ tel que $d_{g}(R, P), d_{g}(R, Q) \geq \beta d_{g}(P, Q)(\beta>0)$ et une constante $c=c\left(c_{0}, c_{\Omega}, \beta, d\right) \geq 1$.

Preuve: On pourra appliquer le Théorème 5.3 (variante des Remarques 5.4) avec $F=\{P, Q\}, x=R, y=P, \eta=\beta d_{g}(P, Q)$ et $N=1$, en notant que par le Lemme 6.3 chaque point de $F_{\eta}^{\Omega}$ peut être joint à $Q$ par un $c_{1}$-arc de John dans $\Omega, c_{1}=c_{1}\left(c_{0}, c_{\Omega}, \beta, d\right)$.

Remarque 6.8. En fait — sous les mêmes hypothèses sur $P, Q$ et $R$ - on voit que plus précisément

$$
c^{-1} G_{P}(Q) \pi_{Q}(R) \leq G_{P}(R) \leq c G_{P}(Q) \pi_{Q}(R)
$$

avec $c=c\left(c_{0}, c_{\Omega}, \beta, d\right)>0$, si $\pi_{Q}$ désigne le potentiel d'équilibre dans $\Omega$ de $B\left(Q, \frac{1}{2} \delta(Q)\right)$. La minoration découle facilement du principe de Harnack (pour $R \in \partial B\left(Q, \frac{1}{2} \delta(Q)\right)$ ) et du principe du maximum.

Corollaire 6.9 (Principe de Harnack au bord le long d'un arc uniforme). Soient $\Gamma$ un arc $c_{1}$-uniforme joignant les points $P$ et $Q$ dans $\Omega$ et $0<$ $\varepsilon_{0} \leq 1$. Pour $A \in \Gamma$ tel que $P, Q \notin B\left(A, \varepsilon_{0} \delta(A)\right)$ on a

$$
G(P, Q) \leq C_{1} \delta(A)^{d-2} G(P, A) \pi_{A}(Q) \leq C_{2} \delta(A)^{d-2} G(P, A) G(A, Q)
$$

où $C_{j}=C_{j}\left(c_{\Omega}, c_{1}, \varepsilon_{0}, d\right)$ et où $\pi_{A}$ désigne le potentiel d'équilibre dans $\Omega$ de $B\left(A, \frac{\varepsilon_{0}}{2} \delta(A)\right)$.

Preuve: On a

$$
c^{-1} G\left(A^{\prime}, A\right) \pi_{A}(P) \pi_{A}(Q) \leq G(P, A) \pi_{A}(Q) \leq c G\left(A^{\prime}, A\right) \pi_{A}(P) \pi_{A}(Q)
$$

si $A^{\prime} \in \partial B\left(A, \frac{\varepsilon_{0} \delta(A)}{2}\right.$ ) pour une constante $c=c(d)$ (principe du maximum et Harnack). On peut donc supposer (par symétrie) que $A$ appartient à la moitié de $\Gamma$ qui contient $Q$. Le sous arc $\Gamma^{\prime}=\widehat{Q A}$ est un $c_{1}$-arc de John (unilatère) et

$$
d_{g}(P, Q) \geq c_{1} \operatorname{long}(\Gamma) \geq c_{1} d_{g}(Q, A) \geq \frac{c_{1}}{1+\varepsilon_{0}^{-1}} \delta(Q)=\varepsilon_{1} \delta(Q)
$$

(en notant que $\delta(Q) \leq \delta(A)+d(Q, A) \leq\left(1+\varepsilon_{0}^{-1}\right) d(Q, A)$ ).

Aussi $d_{g}(P, A) \geq \varepsilon_{0} \delta(A) \geq \varepsilon_{0} c_{1} d_{g}(Q, A)$. La Proposition 6.7 (cf. la Remarque 6.8) appliquée à l'arc $\Gamma^{\prime}$ donne alors le corollaire.

${ }^{(4)} \mathrm{D}$ 'après le Lemme 6.3, pour $P, Q \in \Omega$ donnés, il existe un tel $\operatorname{arc} \Gamma$ avec $c_{0}=$ $c_{0}\left(\beta, c_{\Omega}\right)$ si $d_{g}(P, Q) \leq \beta \delta(Q)$. 
Observons ici qu'un sous-arc "pas trop petit" d'un arc uniforme dans $\Omega$ est aussi uniforme. Une preuve de l'énoncé (certainement standard) suivant est incluse pour la commodité du lecteur.

Lemme 6.10. Soient $\Gamma$ un arc $C$-uniforme de $\Omega$ et $X, Y \in \Gamma$ tels que $d(X, Y) \geq \varepsilon \delta(X), 0<\varepsilon<1$. Le sous-arc $\Gamma^{\prime}$ délimité par $X$ et $Y$ est $C^{\prime}$ uniforme dans $\Omega$ pour une constante $C^{\prime}=C^{\prime}(\varepsilon, C)>0$.

Preuve: D'abord $d(X, Y) \geq \varepsilon \delta(Y)-\varepsilon|\delta(X)-\delta(Y)| \geq \varepsilon \delta(Y)-\varepsilon d(X, Y)$ d'où $d(X, Y) \geq \frac{\varepsilon}{1+\varepsilon} \delta(Y)$. Donc $d(X, Y) \geq \varepsilon^{\prime} \max \{\delta(X), \delta(Y)\}$ où $\varepsilon^{\prime}=\frac{\varepsilon}{1+\varepsilon}$.

Notant $P$ et $Q$ les extrémités de $\Gamma-X$ étant entre $P$ et $Y$ le long de $\Gamma$ - et $\ell$ la longueur de $\Gamma$, on peut supposer que $X$ appartient à la moitié de $\Gamma$ issue de $P$ (sinon échanger $X$ et $Y$ ). Alors si $\operatorname{long}(\widetilde{P X}) \geq \varepsilon_{1} \ell$ (où $\varepsilon_{1}>0$ sera fixé dans la suite), on a $d_{g}(X, Y) \geq \varepsilon \delta(X) \geq \varepsilon C \varepsilon_{1} \ell$ et a fortiori, $d_{g}(X, Y) \geq C \varepsilon \varepsilon_{1} \operatorname{long}\left(\Gamma^{\prime}\right)$. On règle de même le cas où $Y$ appartient à la seconde moitié de $\Gamma$ (celle d'extrémité $Q$ ) et où $\operatorname{long}(\widehat{Y Q}) \geq \varepsilon_{1} \ell$.

Si long $(\widehat{P X}) \leq \varepsilon_{1} \ell$, distinguons deux cas : (a) si $Y$ appartient à la première moitié de $\Gamma$, on a $d_{g}(X, Y) \geq \varepsilon^{\prime} \delta(Y) \geq C \varepsilon^{\prime} \operatorname{long}\left(\Gamma^{\prime}\right)$ et $\operatorname{long}\left(\Gamma^{\prime}\right) \leq C^{-1} \varepsilon^{\prime-1} d_{g}(X, Y)$, (b) si $Y$ appartient à la deuxième moitié de $\Gamma$ et si $\operatorname{long}(\widehat{Y Q}) \leq \varepsilon_{1} \ell$, on a $d_{g}(X, Y) \geq d_{g}(P, Q)-2 \varepsilon_{1} \ell \geq\left(C-2 \varepsilon_{1}\right) \ell$ et le résultat avec $\varepsilon_{1}=\frac{C}{4}$.

On en déduit l'amélioration suivante du Corollaire 6.9.

Corollaire 6.11 (Principe de Harnack au bord le long d'un arc uniforme). Soient $\Gamma$ un $c_{1}$-arc de John bilatère dans $\Omega$ et $\varepsilon_{0}>0$. Si $X$, $Y, Z$ sont trois points se succédant dans cet ordre sur $\Gamma$ et tels que $X, Z \notin B\left(Y, \varepsilon_{0} \delta(Y)\right), Z \notin B\left(X, \varepsilon_{0} \delta(X)\right)$, on a

$$
G(X, Z) \leq C_{1} \delta(Y)^{d-2} G(X, Y) \pi_{Y}(Q) \leq C_{2} \delta(Y)^{d-2} G(X, Y) G(Y, Q)
$$

où $C_{j}=C_{j}\left(c_{\Omega}, c_{1}, \varepsilon_{0}, d\right), j=1,2$ et où $\pi_{Y}$ désigne le potentiel capacitaire de $B\left(Y, \frac{\varepsilon_{0}}{2} \delta(Y)\right.$.

6.D. Pour finir on va donner deux autres conséquences simples du principe de Harnack au bord qui serviront dans la Partie 8. 
Proposition 6.12. Soit $\Gamma$ un $c_{0}$-arc de John joignant le point $\xi \in \bar{\Omega}_{g}$ au point $X \in \Omega$. Supposons que $d(X, \xi) \geq \frac{1}{2} \delta(\xi)\left(\delta(\xi)=0\right.$ si $\left.\xi \in \partial_{g} \Omega\right)$ et que $\xi, X \notin B\left(x_{0}, r_{0}\right), 0<r_{0} \leq \delta\left(x_{0}\right)$. On a alors

$$
c^{-1} K_{X}(Q) \leq K_{\xi}(Q) \leq c K_{X}(Q)
$$

pour tout $Q \in \Omega$ tel que $d_{g}(Q, \xi), d_{g}(Q, X) \geq \beta d_{g}(\xi, X)$. Ici $K$ est le noyau-Martin de $\Omega$ normalisé en $x_{0}$, et $c$ est une constante $>0$ ne dépendant que de $d, \beta, c_{\Omega}, c_{0}$ et $r_{0}^{-1} \operatorname{diam}_{g}(\Omega)$.

Preuve: On supposera pour alléger $d \geq 3$. Prenons $\xi^{\prime} \in \Gamma$ arbitrairement proche de $\xi$ mais distinct de $\xi$. Par la Proposition 6.7 (cf. Remarque 6.8) on a avec une constante $c=c\left(c_{\Omega}, \beta, c_{0}, d\right)$

$c^{-1} \delta(X)^{d-2} G\left(\xi^{\prime}, X\right) G(X, Q) \leq G\left(\xi^{\prime}, Q\right) \leq c \delta(X)^{d-2} G\left(\xi^{\prime}, X\right) G(X, Q)$ si $d_{g}(Q, \xi), d_{g}(Q, X) \geq \beta d_{g}(\xi, X)$. En divisant par $G\left(\xi^{\prime}, x_{0}\right)$, on obtient (6.1) $c^{-1} \delta(X)^{d-2} K_{\xi^{\prime}}(X) G(X, Q) \leq K_{\xi^{\prime}}(Q) \leq c \delta(X)^{d-2} K_{\xi^{\prime}}(X) G(X, Q)$.

En faisant $Q=x_{0}$, on a

$$
c^{-1} \delta(X)^{d-2} K_{\xi^{\prime}}(X) G\left(X, x_{0}\right) \leq 1 \leq c \delta(X)^{d-2} K_{\xi^{\prime}}(X) G\left(X, x_{0}\right)
$$

où $c$ dépend de plus de $r_{0} \times \operatorname{diam}_{g}(\Omega)^{-1}$. En divisant par ces dernières les inégalités (6.1) on a bien

$$
c^{-2} K_{X}(Q) \leq K_{\xi^{\prime}}(Q) \leq c^{2} K_{X}(Q),
$$

où $c=c\left(c_{\Omega}, c_{0}, \beta, d, r_{0}^{-1} \operatorname{diam}_{g}(\Omega)\right)$. Il ne reste plus qu'à faire tendre $\xi^{\prime}$ vers $\xi$ pour obtenir le résultat.

Corollaire 6.13. Soient $\xi \in \partial_{g} \Omega, y \in \Omega \backslash B\left(x_{0}, r_{0}\right)$ (où $\bar{B}\left(x_{0}, r_{0}\right) \subset \Omega$ ) tels que $r:=\delta(y) \geq c d_{g}(\xi, y)$ et soit $h$ harmonique positive sur $\Omega$, nulle sur $\partial_{g} \Omega \backslash \mathbb{T}$ où $\mathbb{T}=\partial_{g} \Omega \cap \bar{B}_{g}\left(\xi, c^{-1} r\right)$. Alors

$$
C^{-1} \frac{h(x)}{h\left(x_{0}\right)} \leq \frac{G_{y}(x)}{G_{y}\left(x_{0}\right)} \leq C \frac{h(x)}{h\left(x_{0}\right)}
$$

pour tout $x \in \Omega$ tel que $d_{g}(x, \mathbb{T}) \geq$ cr et $d_{g}(x, y) \geq c r$. Ici $C$ est une constante ne dépendant que de $d, c_{\Omega}, c$ et $\operatorname{diam}_{g}(\Omega) / r_{0}$.

Par la représentation de Martin $h$ est barycentre des $K_{z}, z \in \mathbb{T}:=$ $\bar{B}_{g}\left(\xi, c^{-1} r\right) \cap \partial_{g} \Omega$ relativement à une mesure de Borel positive finie sur $\mathbb{T}$. Il suffit donc de vérifier l'assertion du corollaire pour $h=K_{z}, z \in \mathbb{T}$.

Or, $d_{g}(z, y) \leq c^{-1} r+d_{g}(\xi, y) \leq 2 c^{-1} \delta(y)$. Il existe donc un $c_{1}$-arc de John joignant $z$ à $y$ dans $\Omega, c_{1}=c_{1}\left(c_{\Omega}, c\right)$ (d'après le Lemme 6.3). Par la Proposition 6.12, on a donc que pour $x$ comme dans l'énoncé $c_{2}^{-1} K_{z}(x) \leq$ $\frac{G_{y}(x)}{G_{y}\left(x_{0}\right)} \leq c_{2} K_{z}(x)$. 
Corollaire 6.14. Soient $x_{0} \in \Omega, r_{0}<\delta\left(x_{0}\right)$ et $\varepsilon_{1}>0$. Si $x \in \Omega \backslash$ $B\left(x_{0}, r_{0}\right), \xi \in \partial_{g} \Omega$ sont tels que $\delta(x) \geq \varepsilon_{1} d_{g}(\xi, x)$, où $0<\varepsilon_{1}<1$, alors pour tout $y \in \Omega \backslash B\left(x, \varepsilon_{1} \delta(x)\right)$ on a

$$
K_{x}(y) \leq c K_{\xi}(y)
$$

pour une constante $\left.c=c\left(\Omega, \varepsilon_{1}, x_{0}, r_{0}\right)\right)>0$.

Par le principe du maximum, il suffit de montrer (6.2) pour $y \in$ $\partial B\left(x, \varepsilon_{1} \delta(x)\right)$. Mais l'inégalité découle dans ce cas de la Proposition 6.12.

\section{Extension à des opérateurs elliptiques du second ordre plus généraux}

7.A. Tout ce qui précède s'étend sans difficulté particulière et à l'aide d'arguments bien connus, à des opérateurs elliptique à structure divergence sur $\Omega$ de la forme

$$
L_{0} u=\sum_{1 \leq i, j \leq d} \partial_{i}\left(a_{i j} \partial_{j} u\right)
$$

où $\left\{a_{i j}\right\}_{1 \leq i, j \leq d}$ est une matrice symétrique positive à coefficients réels mesurables et uniformément bornés sur $\Omega$ tels que

$$
\varepsilon_{0} \sum_{1 \leq i \leq d} \xi_{i}^{2} \leq \sum_{1 \leq i, j \leq d} a_{i j}(x) \xi_{i} \xi_{j} \leq \varepsilon_{0}^{-1} \sum_{1 \leq i \leq d} \xi_{i}^{2}
$$

pour tout $(x, \xi) \in \Omega \times \mathbb{R}^{d}$ et pour une constante $\varepsilon_{0}>0$ convenable. On se contentera ici de renvoyer aux méthodes de [CFMS], [An3] (voir aussi [An1] pour des opérateurs à structure non divergence). On pourrait d'ailleurs rajouter à $L_{0}$ un terme d'ordre 0 de signe variable (moyennant quelques précautions) et $L^{p}$ pour un $p>d / 2$.

Un point important est que contrairement au cas où $\Omega$ est lipschitzien [An1], la symétrie de $L_{0}$ joue un rôle essentiel pour étendre les arguments de la Section 4 (passage au principe de Harnack au bord fort) et la question se pose de savoir si on peut se passer de cette hypothèse de symétrie apparemment artificielle. Par contre, pour $N=1$ la symétrie n'est pas nécessaire pour l'extension des résultats précédents, les résultats de la Section 4 devenant superflus.

On va remarquer ici que sous des hypothèses supplémentaires sur $\Omega$, on peut se débarasser de l'hypothèse de symétrie en combinant [An7] et ce qui précède. Rappelons ([An4]) que $\Omega$ est dit uniformément régulier s'il existe $\beta>0$ tel que pour tout $a \in \partial \Omega$ et tout $r>0$, la mesure harmonique classique (i.e. relative au Laplacien) de $B(a, r) \cap \Omega^{c}$ dans la boule $B(a, r)$ est supérieure ou égale à $\beta$ sur $\partial B(a, r / 2) ; \Omega$ est dit alors 
$\beta$-uniformément régulier. Si cette condition a lieu pour un $a \in \partial \Omega$ donné et un $\beta>0$ on dira que $\Omega$ est $\beta$-uniformément régulier en $a$, ou encore que $\mathbb{R}^{d} \backslash \Omega$ est de densité capacitaire supérieure ou égale à $\beta$ en $a$.

Théorème 7.1. Soit $\Omega$ un ouvert borné, uniformément régulier et de type John dans $\mathbb{R}^{d}$. Soit $L$ un opérateur de la forme

$$
L(u)=L_{0}(u)+B \cdot \nabla u+\alpha u
$$

où $B$ est un champ de vecteurs mesurable sur $\Omega$ tel que $\delta^{1-\varepsilon_{0}}|B| \leq C$ sur $\Omega^{(5)}$ pour des constantes $C>0, \varepsilon_{0}>0$ et où $\alpha$ est une fonction mesurable négative sur $\Omega$ telle que $\delta^{2-\varepsilon_{0}}|\alpha| \leq C$. Alors l'identité de $\Omega$ se prolonge en une application continue surjective $\pi: \widehat{\Omega}_{L} \rightarrow \bar{\Omega}^{g}$ où $\widehat{\Omega}_{L}$ désigne le compactifié de Martin de $\Omega$ relativement à $L$. Si $\xi \in \partial_{g} \Omega$ est John d'ordre $\leq N$, l'ensemble des points minimaux $\zeta$ tels que $\pi(\zeta)=\xi$ est fini de cardinal majoré par $N$.

Démonstration: Par le Théorème 9.1' et la Proposition 8.4 de [An7], la $L$-compactification de Martin s'identifie canoniquement à celle relative à $L_{0}$. D'où le résultat par les remarques précédentes, et en fait un résultat plus précis que celui annoncé. Notons que par le Théorème 9.1' de [An7] la fonction de Green de $L$ est uniformément équivalente à celle de $L_{0}$.

En fait on peut à l'aide du Théorème 9.1' de [An7] étendre tous les résultats des Sections 4 et 5 à l'opérateur $L$ de l'énoncé précédent (au lieu du Laplacien ordinaire) à condition de se limiter à des domaines bornés et uniformément réguliers. Pour le voir montrons un analogue du Théorème 5.3. On suppose ici que $\Omega$ est un domaine borné dans $\mathbb{R}^{d}$ et on suppose toujours que $L_{0}, B$ et $\alpha$ vérifient dans $\Omega$ les mêmes hypothèses que dans le Théorème 7.1 précédent.

Théorème 7.2. Soit $R$ un réel, $0<R \leq 1$, et soient $a \in \partial \Omega$ et $A_{1}, \ldots, A_{m}$ des points de $\partial B(a, R) \cap \Omega$ tels que chaque point $z \in B\left(a, \frac{3}{4} R\right) \cap \Omega$ puisse être joint à au moins l'un des $A_{j}$ par un $c_{0}$-arc de John de $\Omega$ issu de $z$. Supposons aussi que $\Omega$ est $\varepsilon$-uniformément régulier en chaque point de $\partial \Omega \cap B\left(a, \frac{3}{4} R\right)$, pour un $\varepsilon>0$.

Alors pour toute fonction L-harmonique positive $h$ sur $\Omega$ nulle sur $\partial \Omega \cap B\left(a, \frac{3}{4} R\right)$, on $a$

$$
h(x) \leq C_{1} R^{d-2} \sum_{1 \leq j \leq m} h\left(A_{j}\right) G_{A_{j}}(x)
$$

pour tout $x \in \partial B\left(a, \frac{R}{2}\right) \cap \Omega$. Ici $G$ désigne la fonction de Green de $L$ dans $\Omega$ et $C_{1}=C_{1}\left(d, C, \varepsilon_{0}, \varepsilon, c_{0}\right)>0$.

\footnotetext{
${ }^{\left.{ }^{5}\right)}$ Comme précédemment $\delta=\delta_{\Omega}$ désigne la distance au bord dans $\Omega$.
} 
Rappelons que quitte à modifier $c_{0}$ peut supposer que $d\left(A_{j}, A_{k}\right) \geq$ $\frac{c_{0}}{4} R$ pour $j \neq k$ [cf. la Remarque $2.2(2)$ ] et aussi $\delta\left(A_{j}\right) \geq \frac{1}{4} c_{0} R$. On suppose dans la suite que ces inégalités sont vérifiées.

Convenons de noter, si $\gamma:[\alpha, \beta] \rightarrow \bar{\Omega}$ est un $c_{0}$-arc de John de $\Omega$ issu de $\gamma(\alpha)$,

$$
\begin{aligned}
V_{\gamma} & =\bigcup_{\alpha \leq t \leq \beta} B\left(\gamma(t), \frac{c_{0}}{4} \operatorname{long}\left(\gamma_{[\alpha, t]}\right)\right) \\
& =\left\{z \in \Omega ; \exists t \in[\alpha, \beta] \text { tel que } d(z, \gamma(t))<\frac{c_{0}}{4} \operatorname{long}\left(\gamma_{[\alpha, t]}\right)\right\} .
\end{aligned}
$$

L'ensemble $V_{\gamma}$ est un ouvert qu'on peut appeler le $c_{0} / 4$ cône de John associé à $\gamma$. Soit $U$ la réunion des $c_{0} / 4$ cônes de John $V_{\gamma}$ lorsque $\gamma$ parcourt l'ensemble des $c_{0}$-arcs de John de $\Omega$ joignant un point de $B\left(a, \frac{3}{4} R\right) \cap$ $\Omega$ à l'un des $A_{j}$. On vérifie aisément que $U$ est $c$-John pour l'ensemble de pôles $\left\{A_{j}\right\}_{1 \leq j \leq m}$ et que $B\left(a, \frac{3}{4} R\right) \cap \Omega \subset U \subset B\left(a, c^{-1} R\right) \cap \Omega$ pour un $c=c\left(d, c_{0}\right)>0$ assez petit.

En général, $U$ n'est pas nécessairement uniformément régulier et il faut encore modifier $U$ pour passer à un ouvert $V$ à la fois John (relativement aux $A_{j}$ ) et uniformément régulier. Considérons les cubes de Whitney suivants : notons $R^{\prime}=c \frac{R}{4 \sqrt{d}}$ et disons qu'un cube $Q$ est bon s'il est maximal parmi ceux de la forme $Q=\prod_{1 \leq j \leq d}\left[\frac{k_{j}}{2^{\nu}} R^{\prime}, \frac{k_{j}+1}{2^{\nu}} R^{\prime}\right], k_{j} \in \mathbb{Z}$, $\nu$ entier $\geq 4$, et vérifiant $2 Q \subset U, Q \backslash B\left(a, \frac{5}{8} R\right) \neq \emptyset$ - où $2 Q$ est le cube de même centre que $Q$ et homothétique de $Q$ dans le rapport 2-. Notons qu'un bon cube contient au plus un $A_{j}$ et que les bons cubes recouvrent $U \backslash B\left(a, \frac{5}{8} R\right)$.

À chaque bon cube $Q$ associons un sous cube (fermé) $Q^{\prime}$ de la façon suivante : si le cube $Q_{0}$ de même centre que $Q$ et homothétique de celui-ci dans le rapport $\frac{1}{9}$ est tel que $2 Q_{0}$ ne contienne aucun $A_{j}$, on prend $Q^{\prime}=Q_{0}$. Sinon $Q^{\prime}$ est l'un des sous cubes obtenus en divisant $Q$ en $9^{d}$ cubes de même taille, $Q^{\prime}$ étant de plus disjoint de $2 Q_{0}$ et $Q^{\prime} \subset 7 Q_{0}$.

Soit alors $V$ l'ouvert obtenu en enlevant à $U$ tous les cubes $Q^{\prime}$ associés aux bons cubes. On peut alors vérifier que : (a) $V$ est $c^{\prime}$-John d'ensemble de pôles $\left\{A_{j}\right\}_{1 \leq j \leq m}, c^{\prime}=c^{\prime}(c, d)>0$ et (b) $V$ est $\beta$-uniformément régulier pour une constante $\beta=\beta\left(d, \varepsilon, c_{0}\right)>0$. Le (b) découle du Lemme 7.4 ci-dessous; la preuve facile — mais fastidieuse - du (a) sera omise ici.

Maintenant par ce qui précède, les résultats de [An7] (cf. la Remarque 7.3 ci-dessous) disent que $L$ et $L_{0}$ ont dans $V$ des fonctions de Green $G$ et $G_{0}$ équivalentes, i.e. $C_{2}^{-1} G_{0} \leq G \leq C_{2} G_{0}$ dans $V \times V$ 
avec $C_{2}=C_{2}\left(d, C, \varepsilon_{0}, c_{0}, \varepsilon\right)$. Il s'ensuit qu'il existe $h_{0}$, fonction $L_{0}$ harmonique sur $V$, nulle sur $\partial V \cap B\left(a, \frac{5}{8} R\right)$ telle que $C_{2}^{-2} h_{0} \leq h \leq C_{2}^{2} h_{0}$ dans $V$. Comme $L_{0}$ est symétrique, on a d'après le Théorème 4.4 (étendu à $\left.L_{0}\right)$,

$$
h_{0}(x) \leq c_{4} R^{d-2} \sum_{1 \leq j \leq m} h_{0}\left(A_{j}\right) G_{0}\left(x, A_{j}\right)
$$

pour tout $x \in \partial B\left(a, \frac{R}{2}\right) \cap \Omega$ avec $c_{4}=c_{4}\left(d, C, \varepsilon, \varepsilon_{0}, c_{0}\right)$. Donc finalement

$$
h(x) \leq c_{4} C_{2}^{4} R^{d-2} \sum_{1 \leq j \leq m} h\left(A_{j}\right) G\left(x, A_{j}\right)
$$

pour tout $x \in \partial B\left(a, \frac{R}{2}\right) \cap \Omega$.

Remarque 7.3. Dans [An3], le Théorème 9.1' est établi pour un domaine $\varepsilon$-uniformément régulier et $c_{0}$ John par rapport à un pôle. Mais le résultat et la preuve s'étendent au cas d'un ouvert qui est John par rapport à une famille de $m$ points de $\Omega$ (avec une constante de comparaison ne dépendant que de $m, c_{0}$, $\varepsilon$ et des constantes de description des opérateurs elliptiques considérés); on observera que le Théorème XX de [An3] s'étend sans difficulté particulière si on remplace la distance $d(m)=d(O, m)$ au point $O \in M$ par $d(m):=\inf \left\{d\left(O_{j}, m\right) ; 1 \leq\right.$ $j \leq N\}$, où $\left\{O_{j}\right\}_{1 \leq j \leq N}$ est une famille finie de points de référence dans $M$.

Lemme 7.4. Soit $K \subset \partial U$ un fermé tel que $\mathbb{R}^{d} \backslash U$ soit de densité capacitaire $\geq \varepsilon$ en chaque $a \in K$. Soit $\left\{C_{i}\right\}_{i \in I}$ une collection de cubes fermés contenus dans $U$ et tels que $V:=U \backslash \bigcup_{i \in I} C_{i}$ soit ouvert et :

(i) chaque cube $C_{i}$ est de côté $\ell\left(C_{i}\right) \geq \varepsilon \delta\left(x_{i}\right)$, où $x_{i}$ est le centre de $C_{i}\left(\right.$ ici $\left.\delta=\delta_{U}\right)$,

(ii) pour chaque $x \in U$ tel que $\delta(x) \leq \varepsilon d(x ; K)$, il existe $i \in I$ tel que $d\left(x, x_{i}\right) \leq \varepsilon^{-1} \delta(x)$ et $\ell\left(C_{i}\right) \geq \varepsilon \delta(x)$.

Alors, $V$ est uniformément régulier pour une constante $\alpha=\alpha(d, \varepsilon)>0$.

Remarque 7.5. La condition (i) entraîne que pour chaque $a \in C_{i}$ on a $\delta(a) \leq \sqrt{d} \ell\left(C_{i}\right)+\delta\left(x_{i}\right) \leq\left(\sqrt{d}+\varepsilon^{-1}\right) \ell\left(C_{i}\right)$ et $\ell\left(C_{i}\right) \geq \varepsilon^{\prime} \delta(a)$ si $\varepsilon^{\prime}:=$ $\left(\sqrt{d}+\varepsilon^{-1}\right)^{-1}$.

Preuve: Prenons $a \in \Phi:=\partial V, \rho>0$ et notons $B=B(a, \rho)$. Il suffira de montrer que la mesure harmonique $\mu$ de $\Phi \cap B$ dans $B$ en un point $b \in$ $\partial B\left(a, \varepsilon_{1} \rho\right) \cap V$ est $\geq c(d, \varepsilon)>0$ pour un $\varepsilon_{1} \in\left(0, \frac{1}{4}\right)$ à choisir en fonction de $\varepsilon$. En effet, on a alors pour $d\left(a, b^{\prime}\right)=\frac{1}{2} \rho, \mu\left(b^{\prime}\right) \geq c(d, \varepsilon) \times \frac{2^{d-2}-1}{\varepsilon_{1}^{2-d}-1}$ si $d \geq 3$ (resp. $\geq c(d, \varepsilon) \frac{\log 2}{\left|\log \varepsilon_{1}\right|}$ si $\left.d=2\right)$. 
Si $a \in K$, ou plus généralement si $d(b, K) \leq \frac{\rho}{4}$, il n'y a rien à démontrer d'après l'hypothèse sur la densité capacitaire en chaque $a^{\prime} \in$ $K$ de $\mathbb{R}^{d} \backslash U \subset \mathbb{R}^{d} \backslash V$. Si $a \in \partial U \backslash K$ et $d(b, K)>\frac{\rho}{4}$, on a puisque $\delta(b) \leq \varepsilon_{1} \rho$

$$
B\left(b, 2 \varepsilon^{-1} \delta(b)\right) \subset B\left(a, 2\left(\varepsilon^{-1}+1\right) \varepsilon_{1} \rho\right) \subset B
$$

si $2\left(\varepsilon^{-1}+1\right) \varepsilon_{1} \leq 1$; alors, par l'hypothèse (ii), $B$ contient le centre $x_{i}$ d'un $C_{i}$ de côté $\ell\left(C_{i}\right) \geq \varepsilon \delta(b)$. La mesure harmonique dans $B\left(b, 2 \varepsilon^{-1} \delta(b)\right)$ de $C_{i}$ est $\geq \alpha(\varepsilon)$ au point $b$ et a fortiori $\mu(b) \geq \alpha$.

Ainsi $\mathbb{R}^{\bar{d}} \backslash V$ est de densité capacitaire $\geq \alpha^{\prime}(\varepsilon)>0$ en tout $a \in \partial U$. Considérons maintenant le cas où $a \in \partial C_{i}$ pour un $i \in I$. Si $\delta(a) \leq \frac{\rho}{4}$ on a $B(a, \rho) \supset B\left(a^{\prime}, \frac{\rho}{4}\right), a^{\prime} \in \partial U$ et, puisque $\mathbb{R}^{d} \backslash V$ est de densité capacitaire $\geq \alpha^{\prime}(\varepsilon)$ en $a^{\prime}$, on a $\mu(b) \geq \alpha^{\prime}$. Si $\rho \leq 4 \delta(a)$ (et $a \in \partial C_{i}$ pour un $i \in I$ ), l'hypothèse (i) et la Remarque 7.5 donnent que $\ell\left(C_{i}\right) \geq$ $\varepsilon^{\prime} \delta(a) \geq \frac{\varepsilon^{\prime}}{4} \rho$; donc $\mu(b)$ est minoré par la mesure harmonique dans $B(a, \rho)$ d'un cube contenant $a$ et de côté $\geq \frac{\varepsilon^{\prime}}{4} \rho$, évaluée en un point de $\partial B\left(a, \varepsilon_{1} \rho\right)$. Il s'ensuit que $\mu(b) \geq C_{1}$ avec $C_{1}=C_{1}(d, \varepsilon)>0$.

\section{Une version du théorème de limite radiale de Littlewood}

Pour alléger, on se limite dans cette partie et la suivante à la théorie du potentiel par rapport au Laplacien. Les résultats s'étendent sans difficulté au cas de la théorie du potentiel associée à un opérateur elliptique $L_{0}$ comme en Section 7 (et donc aussi à un opérateur $L$ comme dans le Théorème 7.1 dans le domaine $\Omega$ envisagé lorsque celui-ci est uniformément régulier).

8.A. Soit $\Omega$ un domaine borné et intérieurement uniforme de $\mathbb{R}^{d}$ et soit $\partial_{g} \Omega=\bar{\Omega}_{g} \backslash \Omega$ sa frontière accessible. Fixons un point de réference $x_{0} \in \Omega$ et un réel $r_{0}>0$ tels que $B\left(x_{0}, 2 r_{0}\right) \subset \Omega$.

On se donne une partie $E$ de $\partial_{g} \Omega$ et un champ non tangentiel de base $E$, c'est à dire ici la donnée d'une partie borélienne (ou seulement analytique au sens de Souslin, cf. [Bou]) $\Gamma$ de $\Omega \backslash B\left(x_{0}, r_{0}\right)$ et d'une application $\pi: \Gamma \rightarrow E$ telles que

(i) $\pi$ est lipschitzienne ( $\Omega$ et $E$ étant munis de $d_{g}$ ),

(ii) $\delta(x) \geq c_{1} d_{g}(x, \xi)$ pour $x \in \Gamma, \xi=\pi(x) \in E$ et une constante $c_{1}>0$,

(iii) $\xi \in \overline{\pi^{-1}(\xi)}$ (adhérence au sens de $d_{g}$ ) pour tout $\xi \in E$.

Puisque $\pi$ est continue et $\Gamma$ analytique, l'ensemble $E$ est aussi une partie analytique de $\partial_{g} \Omega$. Une façon naturelle de produire de tels champs 
est donnée par la notion suivante, due pour l'essentiel à Zhao [Zha] et à l'origine de la définition ci-dessus.

Exemple 8.1 (Les champs de rayons non tangentiels). Soit $E \subset \partial_{g} \Omega$ borélien et soit $\gamma: E \times(0,1) \rightarrow \Omega \backslash B\left(x_{0}, r_{0}\right)$ une application Borel-mesurable vérifiant les conditions suivantes pour un $c \geq 1$ :

(i) $d(\gamma(\zeta, t), \partial \Omega) \geq c^{-1} d_{g}(\gamma(\zeta, t), \zeta)$ pour $(\zeta, t) \in E \times(0,1)$,

(ii) $\left|\gamma(\zeta, t)-\gamma\left(\zeta^{\prime}, s\right)\right| \geq c^{-1} d_{g}\left(\zeta, \zeta^{\prime}\right)$ pour $\zeta, \zeta^{\prime} \in E$, et $t, s \in(0,1)$,

(iii) $\zeta=\lim _{t \rightarrow 0} \gamma(\zeta, t)$ pour tout $\zeta \in E$.

Si on pose $\Gamma=\{\gamma(\xi, t) ; \xi \in E, t \in(0,1)\}$ et si on définit $\pi: \Gamma \rightarrow E$ par la formule $\pi(\gamma(\xi, t))=\xi$, alors le couple $(\Gamma, \pi)$ est un champ non tangentiel de base $E$.

8.B. Notons $\omega\left(x_{0},.\right)$ la mesure harmonique dans $\Omega$ de $x_{0}$ (relativement à la compactification géodésique $\bar{\Omega}_{g}$ ou ce qui revient au même à la compactification de Martin $\widehat{\Omega}$ ). L'existence de $\omega_{x_{0}}$ est établie en général dans $[\mathbf{B r} \mathbf{1}],[\mathbf{B r 2}],[\mathbf{B r} 3]$ et il est bien connu (cf. [Br3]) que cette mesure coïncide avec l'unique mesure de probabilité $\mu_{x_{0}}$ sur $\partial_{g} \Omega$ telle que $\int K_{\zeta}(x) d \mu_{x_{0}}(\zeta)=1, \forall x \in \Omega$-si $K$ désigne le noyau Martin de $\Omega$ relatif au point de normalisation $x_{0}$ - Donc $\int_{\partial_{g} \Omega} K_{\zeta}(x) \omega\left(x_{0}, d \zeta\right)=1$ pour tout $x \in \Omega$.

Pour $L$ partie compacte de $\Omega$ on notera aussi $\omega\left(x_{0}, L\right)$ la mesure harmonique de $L$ dans $\Omega$ au point $x_{0}$. C'est aussi la valeur en $x_{0}$ de la réduite $\widehat{R}_{1}^{L}$ (relative à $\Omega$ ) (ref. e.g. $[\mathbf{B r} 4],[\mathbf{H e r}]$ ).

8.C. On se propose de montrer, dans le prolongement des méthodes de $[\mathbf{A C}, \S \mathrm{A} .1]$, que pour toute $s$ surharmonique positive dans $\Omega$ la limite $\lim _{x \in \pi^{-1}(\xi), x \rightarrow \xi} s(x)=\ell_{\xi}$ existe pour $\omega_{x_{0}}$-presque tout $\xi \in E$. Le point crucial est d'établir l'inégalité de projection de type Doob ([Doo], [LMT]) suivante. La preuve qui suit s'inspire toujours de [Doo] (cf. aussi l'exposé dans $[\mathbf{L M T}]$ ) et est parallèle à celle de [AC, $\S \mathrm{A} .1]$; néanmoins un nouvel argument permet ici d'éviter l'hypothèse d'uniforme régularité de $\Omega$. Pour d'autres méthodes (et des résultats antérieurs) voir [Da2] pour $\Omega$ Lipschitzien et $[\mathbf{Z h a}]$ pour $\Omega$ NTA.

Théorème 8.2. Il existe $C>0$ tel que pour tout compact $L \subset \Gamma$, on a :

$$
\omega\left(x_{0}, \pi(L)\right) \leq C \omega\left(x_{0}, L\right) .
$$

Preuve: Fixons (comme dans $[\mathbf{L M T}]$ ) une mesure de Borel positive $\mu$ sur $L$ dont la projection $\pi(\mu)$ soit exactement la restriction de $\omega\left(x_{0},.\right)$ à $\pi(L)$ (l'existence de $\mu$ se déduit facilement du théorème de prolongement de Hahn-Banach appliqué à $f \mapsto \int g(x) \omega\left(x_{0}, d x\right), f=g \circ \pi, g \in$ 
$C(\pi(L))$, voir $[\mathbf{L M T}])$. Considérons le potentiel $p(y)=\int_{L} K_{x}(y) d \mu(x)$, $y \in \Omega$, (rappelons que $K$ désigne le noyau Martin de $\Omega$ normalisé en $x_{0}$ ) et montrons qu'on a $p \leq c$ sur $L$ pour une constante $c$ indépendante de $L$.

Pour y parvenir on part de la decomposition $p=p_{1}+p_{2}$ où

$$
p_{1}(y)=\int_{L \cap B\left(y, \frac{1}{2} \delta(y)\right)} K_{x}(y) d \mu(x), \quad p_{2}(y)=\int_{L \backslash B\left(y, \frac{1}{2} \delta(y)\right)} K_{x}(y) d \mu(x) .
$$

Pour estimer $p_{2}$ notons que si $d(x, y) \geq \frac{1}{2} \delta(y)$ alors $\delta(x) \leq d(x, y)+$ $\delta(y) \leq 3 d(x, y)$ et $d(x, y) \geq \frac{1}{3} \delta(x)$. Donc, d'après les inégalités de Harnack au bord disponibles ici (voir le Corollaire 6.14), on a, pour $x \in \Gamma$ et $y \in \Omega \backslash B\left(x, \frac{1}{2} \delta(y)\right)$,

$$
K_{x}(y) \leq c K_{\pi(x)}(y)
$$

pour une constante $c=c(\Omega, \Gamma, \pi)>0$.

À partir de (8.1), on obtient

$$
p_{2}(y) \leq c \int_{L} K_{\pi(x)}(y) d \mu(x)=c \int_{\pi(L)} K_{\zeta}(y) \omega\left(x_{0}, d \zeta\right) \leq c,
$$

où on a utilisé la propriété $\pi(\mu)=\omega\left(x_{0},.\right)$ sur $\pi(L)$ et l'identité $\int_{\partial_{g} \Omega} K_{\zeta}(y) \omega\left(x_{0}, d \zeta\right)=1$ (cf. le 8.B).

Pour estimer $p_{1}$, introduisons un domaine borné $V$ contenant la fermeture de $\Omega$ et observons la majoration : $K_{x}(y) \leq c \frac{G_{V}(y, x)}{G\left(x_{0}, y\right)}$ pour $x \in \Gamma \cap$ $B\left(y \frac{1}{2}, \delta(y)\right), G$ (resp. $G_{V}$ ) désignant la fonction de Green de $\Omega$ (resp. $V$ ) - noter que par les inégalités de Harnack $G\left(x_{0}, y\right) \leq c_{d}^{\prime} G\left(x_{0}, x\right)$ - si $c$ a été fixée assez grande. Comme la projection $\pi: \Gamma \rightarrow \partial_{g} \Omega$ est lipschitzienne, on a $G_{V}(x, y) \leq c G_{V}(\pi(y), \pi(x))$ (quitte à agrandir la constante c) - en convenant ici que pour $z, z^{\prime} \in \bar{\Omega}_{g}, G_{V}\left(z, z^{\prime}\right):=G_{V}\left(u, u^{\prime}\right)$ où $u, u^{\prime}$ sont les projections naturelles de $z, z^{\prime}$ dans $\bar{\Omega}$. En effet, $z \mapsto u$ est 1-lipschitzienne de $\left(\bar{\Omega}_{g}, d_{g}\right)$ sur $\bar{\Omega}$ muni de sa métrique ordinaire et $c_{V}^{-1}\left|u-u^{\prime}\right|^{2-d} \leq G_{V}\left(z, z^{\prime}\right) \leq c_{V}\left|u-u^{\prime}\right|^{2-d}$ pour $z, z^{\prime} \in \bar{\Omega}_{g}$ et une constante $c_{V} \geq 1$ (avec des modifications standard si $d=2$ ). Donc,

$$
\begin{aligned}
p_{1}(y) & \leq \frac{c_{1}}{G\left(x_{0}, y\right)} \int_{B\left(y, \frac{1}{2} \delta(y)\right)} G_{V}(y, x) d \mu(x) \\
& \leq \frac{c_{2}}{G\left(x_{0}, y\right)} \int_{\pi\left[B\left(y, \frac{1}{2} \delta(y)\right)\right]} G_{V}(\pi(y), z) \omega\left(x_{0}, d z\right),
\end{aligned}
$$

où $c_{1}$ et $c_{2}$ sont des constantes positives (ne dépendant que de $\Omega, \Gamma$ et $\pi$ ). 
Considérons maintenant la fonction

$$
h: x \mapsto \int_{\pi\left[B\left(y, \frac{1}{2} \delta(y)\right)\right]} G_{V}(\pi(y), z) \omega(x, d z), \quad x \in \Omega .
$$

Cette fonction $h$ est harmonique positive dans $\Omega$ et s'annulle sur $\partial_{g} \Omega$ hors de $\pi\left(\bar{B}\left(y, \frac{1}{2} \delta(y)\right)\right.$, donc sur $\partial_{g} \Omega$ hors de $B_{g}(\pi(y), c \delta(y))$ pour une constante $c>0$ assez grande (puisque $\pi$ est Lipschitzienne). De plus la valeur en $y$ de $h$ est majorée par $G_{V}(\pi(y), y)$ : en effet, pour toute fonction surharmonique $s$ sur $V$ on a $s(y) \geq \int_{\partial_{g} \Omega} s(z) \omega(y, d z)$ par le principe du maximum (ou par la définition même de $\omega$ ). Et $h(y)$ est donc aussi majorée, si $\boldsymbol{d} \geq \mathbf{3}$, par $c G_{V}\left(y^{\prime}, y\right) \leq c^{\prime} G_{y}\left(y^{\prime}\right), y^{\prime} \in \partial B\left(y, \frac{1}{2} \delta(y)\right)$ (avec de nouvelles constantes $c>0$ ); d'où $h\left(y^{\prime}\right) \leq c G_{y}\left(y^{\prime}\right)$ (par Harnack quitte à modifier encore $c$ ). Par les inégalités de Harnack au bord du Corollaire 6.13, on obtient alors que $h\left(x_{0}\right)$ est majoré par $c G\left(x_{0}, y\right)=$ $c G_{y}\left(x_{0}\right), c:=c(\Omega, \Gamma, \pi)>0$.

À ce point on a montré, compte tenu de (8.3) et si $d \geq 3$, que $p(y) \leq c$ pour $y \in L$ et une constante $c=c(\Omega, \Gamma, \pi)$. Le principe du maximum dit qu'en fait on a dans $\Omega$ tout entier

$$
p(y) \leq c \widehat{R}_{1}^{L}(y)
$$

où $\widehat{R}_{1}^{L}$ désigne la réduite (régularisée) dans $\Omega$ de la fonction constante 1 sur $L$, c'est à dire le potentiel capacitaire de $L$ dans $\Omega$.

En prenant maintenant $y=x_{0}$ et en observant que $p\left(x_{0}\right)=\|\mu\|=$ $\omega\left(x_{0}, \pi(L)\right), R_{1}^{L}\left(x_{0}\right)=\omega\left(x_{0}, L\right)$, on obtient finalement la conclusion $\mathrm{du}$ théorème dans le cas $d \geq 3$.

Le cas $d=2$. L'argument ci-dessus semble difficile à adapter pour $d=2$. On peut néanmoins déduire ce cas de celui de la dimension 3 de la manière suivante. Formons $\tilde{\Omega}:=\Omega \times(-2,2)$ et posons $\tilde{x}_{0}=\left(x_{0}, 0\right)$, $\tilde{\Gamma}=\Gamma \times[-1,1], \tilde{\pi}(x, t)=(\pi(x), t)$ pour $(x, t) \in \Gamma$. On montre aisément que $\tilde{\Omega}$ est intérieurement uniforme (pour un résultat général pour les domaines produits voir $[\mathbf{B S t}])$ et que $\partial_{g} \tilde{\Omega}$ s'identifie naturellement à $\left(\partial_{g} \Omega \times[-2,2]\right) \cup(\Omega \times\{-2,2\})$. Aussi $(\tilde{\Gamma}, \tilde{\pi})$ est un champ non tangentiel dans $\tilde{\Omega}$ de base $\tilde{E}:=E \times[-1,1]$. De plus, pour $K$ compact de $\Omega$, $\omega\left(x_{0}, K\right) \geq \tilde{\omega}\left(\tilde{x}_{0}, K \times[-1,1]\right)$ (où $\tilde{\omega}$ désigne la mesure harmonique relative à $\tilde{\Omega}$ ) et, pour $L$ compact de $\partial_{g} \Omega$ on a, comme on va voir, que pour une constante $c>0$ indépendante de $L$

$$
\omega\left(x_{0}, L\right) \leq c \tilde{\omega}\left(\tilde{x}_{0}, L \times[-1,1]\right) .
$$


Appliquant alors le Théorème 8.2 à $\tilde{\Omega}$ et $\tilde{\pi}$, on obtient $\omega\left(x_{0}, \pi(K)\right) \leq$ $C \omega\left(x_{0}, K\right)$ pour tout compact $K \subset \Gamma$ et une constante $C$ indépendante de $K$, soit le Théorème 8.2 pour $d=2$.

La majoration (8.4) peut se déduire d'un théorème de CranstonMcConnell $[\mathbf{C M}]$ propre à la dimension 2. Soient $\left\{X_{t}\right\}$ un mouvement Brownien plan issu de $x_{0}$ et $\left\{Z_{t}\right\}$ un mouvement Brownien réel indépendant. Si $T$ désigne le premier temps de sortie de $X$ de $\Omega$, il existe d'après $[\mathbf{C M}]$ une constante absolue $\alpha>0$ telle que $E\left(T \mid X_{T} \in L\right) \leq$ $\alpha|\Omega|$-où $|\Omega|=\iint_{\Omega} d x d y$ et $X_{T}$ est vu comme point de la frontière accessible $\partial_{g} \Omega$ - Donc $P_{x}\left(T>M \mid X_{T} \in L\right) \leq \frac{\alpha}{M}|\Omega|=\frac{1}{2}$ si $M=2 \alpha|\Omega|$. On en déduit, si $c_{0}:=P\left(\sup _{0 \leq s \leq M}\left|Z_{s}\right| \leq 1\right)$,

$$
\begin{aligned}
& P\left(\sup _{0 \leq s \leq M}\left|Z_{s}\right| \leq 1, T \leq M, X_{T} \in L\right) \\
= & P\left(\sup _{0 \leq s \leq M}\left|Z_{s}\right| \leq 1\right) P\left(T \leq M, X_{T} \in L\right) \\
\geq & \frac{c_{0}}{2} P\left(X_{T} \in L\right) .
\end{aligned}
$$

Ainsi $\tilde{\omega}\left(\tilde{x}_{0}, L \times[-1,1]\right)=P\left(X_{T} \in L, \sup _{s \leq T}\left|Z_{s}\right|<2,\left|Z_{T}\right| \leq 1\right) \geq$ $\frac{c_{0}}{2} P\left(X_{T} \in L\right)=\frac{c_{0}}{2} \omega\left(x_{0}, L\right)$.

Corollaire 8.3. Pour tout borélien $A$ de $\Gamma$, on a $\omega\left(x_{0}, \pi(A)\right) \leq c \omega\left(x_{0}, A\right)$.

En effet $\Gamma$ est une partie analytique de $\mathbb{R}^{d}$ et $\pi(A)$ est donc analytique comme image continue de $A$. En particulier, $\pi(A)$ est $\omega_{x_{0}}$-mesurable (ref. [Bou]). D'autre part il existe un prolongement lipschitzien $\tilde{\pi}$ de $\pi$ sur la fermeture $F$ de $\Gamma$ dans $\Omega$, et $X \mapsto \mathcal{C}(X)=\stackrel{*}{\omega}_{x_{0}}(\tilde{\pi}(X)), X \subset F$, est une capacité de Choquet sur $F$. Par le théorème de capacitabilité de Choquet on a $\omega(\pi(A))=\sup \{\omega(\pi(K)) ; K$ compact $\subset A\}$ et le corollaire résulte alors du Théorème 8.2 .

Théorème 8.4 (Propriété de Littlewood). Pour toute fonction surharmonique positive $s$ dans $\Omega$, la limite "radiale" $\lim _{x \rightarrow \zeta, x \in \pi^{-1}(\zeta)} s(x)=\ell_{\zeta}$ existe et est finie pour $\omega_{x_{0}}$ presque tout $\zeta \in E$.

La preuve montre que comme on doit s'y attendre, $\zeta \mapsto \ell_{\zeta}$ est une densité (sur $E$, relativement à $\omega_{x_{0}}$ ) de la mesure représentative sur $\partial_{g} \Omega$ de la plus grande minorante harmonique de $s$ dans $\Omega$.

Par le théorème de Fatou du 5.8, il suffit de montrer que si $s$ est un potentiel dans $\Omega$ (la plus grande minorante harmonique de $s$ est nulle), on a $\lim _{x \rightarrow \zeta, x \in \pi^{-1}(\zeta)} s(x)=0$ pour $\omega_{x_{0}}$ presque tout $\zeta \in E$. Pour 
un $\varepsilon>0$ fixé, considérons l'ensemble $A=\{x \in \Omega ; s>\varepsilon\}$ et posons $A_{n}=A \cap\left\{x ; \delta(x) \leq \frac{1}{n}\right\}$ pour $n \in \mathbb{N}^{*}$.

Quand on fait croitre $n$ vers $+\infty$, la réduite $\widehat{R}_{1}^{A_{n}}$ décroit vers une fonction harmonique $h \geq 0$ dans $\Omega$. Comme $h \leq \widehat{R}_{1}^{A_{n}} \leq \widehat{R}_{1}^{A} \leq \frac{1}{\varepsilon} s$ et que $s$ est un potentiel, on doit avoir $h=0$ et $\lim _{n \rightarrow \infty} \widehat{R}_{1}^{A_{n}}\left(x_{0}\right)=0$. Du Théorème 8.4 on déduit alors que $\lim _{n \rightarrow \infty} \omega_{x_{0}}\left(\pi\left(A_{n}\right)\right)=0$. Ce qui signifie exactement que pour $\omega_{x_{0}}$ presque tout $\zeta \in \partial_{g} \Omega$, on a $s(x) \leq \varepsilon$ si $x \in$ $\pi^{-1}(\zeta)$ et $\delta(x)<\frac{1}{n}$ pour $n \geq N(\zeta, \varepsilon)$. D'où le Théorème 8.4.

\section{Sur le caractère quasi-métrique de l'inverse du noyau de Naïm}

9.A. Dans $[\mathbf{K V}]$ (voir p. 3488) Kalton et Verbitsky ont soulevé la question de savoir si, étant donnés un domaine de Lipschitz $\Omega$ de $\mathbb{R}^{d}$ de fonction de Green $G$ et un point $x_{0} \in \Omega$, la fonction $d_{\theta}(x, y)=\frac{G\left(x, x_{0}\right) G\left(x_{0}, y\right)}{G(x, y)}$ (l'inverse du noyau de Naïm $\theta$ de $\Omega$ relatif au point de référence $x_{0} \in \Omega$, cf. [Naï]) possède en général la propriété de quasi-métrique, c'est à dire que

$$
d_{\theta}(x, z) \leq C\left(d_{\theta}(x, y)+d_{\theta}(y, z)\right)
$$

pour tout $(x, y, z) \in\left(\Omega \backslash\left\{x_{0}\right\}\right)^{3}$ et une constante $C=C\left(\Omega, x_{0}\right)>0$ convenable. Dans $[\mathbf{K V}]$, Kalton et Verbitsky ont d'ailleurs établi (9.1) pour $\Omega$ assez régulier et dans [An8] (cf. pp. 131-132) une réponse positive à la conjecture (et une extension au cadre des variétés Gromovhyperboliques) était annoncée. Indépendamment W. Hansen [Han] a également établi cette conjecture pour les domaines uniformes et les puissances fractionnaires du Laplacien. Nous renvoyons également à [Han] pour une riche bibliographie sur la question et des applications.

On se propose dans cette section de décrire notre preuve de (9.1) pour un ouvert uniforme ${ }^{(6)}$ ou une variété hyperbolique. Comme celle de Hansen, elle s'appuie fortement sur la propriété de Harnack au bord. La différence est qu'ici on s'appuiera d'autre part très explicitement sur l'hyperbolicité de $\Omega$ pour la métrique quasi-hyperbolique; en particulier la propriété s'étendra à tout opérateur elliptique adapté et faiblement coercif sur une variété hyperbolique (ref. [An6]).

${ }^{(6)}$ On montre ici (9.1) pour $x, y, z \notin B\left(x_{0}, \frac{1}{2} \delta\left(x_{0}\right)\right)$ tels que les boules $B\left(x, \frac{\delta(x)}{4}\right)$, $B\left(y, \frac{\delta(y)}{4}\right), B\left(z, \frac{\delta(z)}{4}\right)$ soient deux à deux disjointes. Les cas restants se traitent facilement par un examen élémentaire indépendant du principe de Harnack au bord. 
9.B. Un cadre général. On se donne un espace métrique complet, géodésique et Gromov-hyperbolique $(M, d)$ (voir e.g. [BS]) et on se donne aussi une fonction $\tilde{G}: M \times M \rightarrow \mathbb{R}_{+}^{*}$ vérifiant le principe de Harnack à l'infini suivant (où $C$ désigne une constante réelle $\geq 1$ ) : pour $Y$ appartenant à un segment géodésique $[X, Z]$ de $M$ (i.e. $[X, Z]$ est un arc de longueur minimum joignant $X$ à $Z$ dans $M)$ on a

$$
C^{-1} \tilde{G}(X, Y) \tilde{G}(Y, Z) \leq \tilde{G}(X, Z) \leq C \tilde{G}(X, Y) \tilde{G}(Y, Z)
$$

où $C=C_{M}$ désigne une constante réelle $>1$ (indépendante des points et du segment géodésique considérés). On suppose aussi que :

(i) $\tilde{G}(x, y) \leq C \tilde{G}\left(x^{\prime}, y^{\prime}\right)$ si $d\left(x, x^{\prime}\right), d\left(y, y^{\prime}\right) \leq 1$ (propriété de Harnack),

(ii) $\frac{1}{C} \leq \tilde{G}(x, y) \leq C$ pour $(x, y) \in M \times M$ et $d(x, y) \leq 1$,

(iii) $\tilde{G}(x, y) \tilde{G}(y, x) \leq C$ pour tout $(x, y) \in M \times M$.

Bien entendu sous l'hypothèse (i) la condition (ii) revient (à modification de la constante $C$ près) à $\frac{1}{C} \leq \tilde{G}(x, x) \leq C$ pour tout $x \in M$. Noter aussi que $(\mathrm{PHI}) \Rightarrow$ (ii) et qu'on ne suppose pas la symétrie de $\tilde{G}$.

On fixe un point de référence $O \in M$ et on pose $: \tilde{\theta}(x, y)=\frac{\tilde{G}(x, y)}{\tilde{G(x, O) \tilde{G}(O, y)}}$ pour $(x, y) \in M$.

Remarque 9.1. Pour les applications à la fonction de Green $G$ d'un opérateur elliptique du second ordre sur une variété hyperbolique $M$ (resp. sur un domaine intérieurement uniforme borné $\Omega$ de $\mathbb{R}^{d}$ ) on prendra $\tilde{G}(x, y)=G(x, y)$ si $d(x, y)>1$ et $\tilde{G}(x, y)=1$ si $d(x, y) \leq 1$ (resp. $\tilde{G}(x, y)=\delta(x)^{d-2} G(x, y)$ si $d(x, y) \geq \frac{1}{2} \delta(x) \wedge \delta(y), \tilde{G}(x, y)=1$ si $d(x, y)<\frac{1}{2} \delta(x) \wedge \delta(y)$, où $\delta=\delta_{\Omega}$ est la distance au bord dans $\left.\Omega\right)$. Dans ce dernier cas, on substituera à la métrique usuelle la métrique pseudo hyperbolique de $\Omega$ (cf. le C plus loin).

Outre (PHI) on utilisera l'ingrédient géométrique suivant ainsi qu'une autre propriété caractéristique de la géométrie hyperbolique explicitée plus loin.

Lemme 9.2. Si $X, Y$ et $Z$ sont des points de $M$ tels que $d(Y, X)=$ $d(Y,[X, Z])$, alors $d(X,[Y, Z]) \leq c$ où $c=c(M)$ est une constante $>0$.

Cette propriété est assez claire si $M$ est l'espace hyperbolique $H_{N}(-1)$. Pour le cas général voir par exemple [An6, 2.6, p. 82].

Comme dans [An8], il découle alors facilement de (ii) et de (PHI) que si $H=H(X, Y)$ est un point du segment geodesique $[X, Y]$ à distance 
minimum du point de référence $O$ fixé dans $M$, on a

$$
\frac{c^{-1}}{\tilde{G}(O, H) \tilde{G}(H, O)} \leq \tilde{\theta}(X, Y) \leq \frac{c}{\tilde{G}(O, H) \tilde{G}(H, O)}
$$

pour une constante $c=c(M, C) \geq 1$. (Voir [An8, fin de la Section 2].)

\section{C.}

1. Propriété de quasi-métrique pour $\tilde{\theta}^{-1}$. Soient maintenant $X, Y, Z \in M$ et soit $K=H(X, Z)$ un point de $[X, Z]$ à distance minimum de $O$. La propriété des "triangles fins" (caractéristique de l'hyperbolicité au sens de Gromov, cf. e.g. [BS] ou $[\mathbf{A n 6}, \S 6])$ dit qu'il existe une constante $R_{0}=$ $R_{0}(M)$ telle que

$$
d(K,[X, Y] \cup[Y, Z])) \leq R_{0}
$$

Donc ou bien $d(K,[X, Y]) \leq R_{0}$ ou bien $d(K,[Y, Z]) \leq R_{0}$. Supposons qu'on soit dans le premier cas et soit $K^{\prime}$ un point de $[X, Y]$ à distance de $K$ majorée par $R_{0}$. Soit aussi $H=H(X, Y)$. Par (9.2) et Harnack (i), on a pour une constante $c_{1}=c_{1}(M, C) \geq 1$,

$$
c^{-1} \tilde{G}\left(O, K^{\prime}\right) \tilde{G}\left(K^{\prime}, O\right) \leq \tilde{\theta}(X, Z)^{-1} \leq c \tilde{G}\left(O, K^{\prime}\right) \tilde{G}\left(K^{\prime}, O\right) .
$$

A l'aide du Lemme 9.2, (i) et (PHI), on voit que $\tilde{G}\left(O, K^{\prime}\right)$ est uniformément de l'ordre de $\tilde{G}(O, H) \times \tilde{G}\left(H, K^{\prime}\right)$ ou encore (par Harnack) du même ordre que $\tilde{G}(O, H) \tilde{G}(H, K)$. Utilisant les équivalences similaires pour $\tilde{G}\left(K^{\prime}, O\right)$ et tenant compte des estimations $(9.2)$ de $\tilde{\theta}(X, Y)$ on obtient

$$
c_{2}^{-1} \frac{\tilde{\theta}(X, Y)}{\tilde{G}(H, K) \tilde{G}(K, H)} \leq \tilde{\theta}(X, Z) \leq c_{2} \frac{\tilde{\theta}(X, Y)}{\tilde{G}(H, K) \tilde{G}(K, H)},
$$

pour une constante $c_{2}:=c_{2}(M, C)$. Utilisant la propriété (iii) on obtient alors

$$
\tilde{\theta}(X, Z) \geq C^{-1} c_{2}^{-1} \tilde{\theta}(X, Y)=c_{3} \tilde{\theta}(X, Y) .
$$

De même si $d(K,[Y, Z]) \leq R_{0}$, on trouve $\tilde{\theta}(X, Z) \geq c_{3} \tilde{\theta}(Y, Z)$. Ainsi dans tous les cas l'inégalité de quasi-métrique suivante a lieu :

(9.4) $\tilde{\theta}^{-1}(X, Z) \leq c^{-1} \max \left\{\tilde{\theta}^{-1}(X, Y), \tilde{\theta}^{-1}(Y, Z)\right\}, \quad c=c(M, C)>0$. 
2. Application aux fonctions de Green d'opérateur elliptiques sur des variétés hyperboliques. Soit $M$ une variété Riemannienne complète de dimension $d \geq 2$ qui est Gromov-hyperbolique et à géométrie bornée au sens que les boules $B_{x}=B\left(x, r_{0}\right)$ sont uniformément bilipschitziennes à la boule unité de $\mathbb{R}^{d}$ pour un $r_{0}>0$ fixé. Plus précisément, on suppose les hypothèses de $[\mathbf{A n} \mathbf{8}$, Chapter 1] remplies. Soit $\mathcal{L}$ un opérateur différentiel du second ordre de la forme $\mathcal{L} u=\operatorname{div}(A \nabla u)+B_{1} \cdot \nabla u+\operatorname{div}\left(B_{2} u\right)+V u$. Ici $A$ est une section de $\operatorname{End}(T(M))$ telle que pour tout $\xi \in T M$, $\langle A \xi, \xi\rangle \geq C^{-1}\|\xi\|^{2}$ et $\left\|A_{x}\right\| \leq C$ où $x$ est le point base de $\xi$ et $C$ un constante $>0$. De plus $B_{1}$ et $B_{2}$ sont deux champs de vecteurs mesurables uniformément bornés sur $M$ et $V$ une fonction mesurable uniformément bornée sur $M$ (on pourrait considérer des conditions moins restrictives du type $\left\|B_{1}\right\|_{L^{p}\left(B_{x}\right)}+\left\|B_{2}\right\|_{L^{p}\left(B_{x}\right)}+\|V\|_{L^{q}\left(B_{x}\right)} \leq C$ avec $p>d$, $q>d / 2$ ). On suppose en outre que $\mathcal{L}$ est faiblement coercif, c'est à dire qu'il existe $\varepsilon_{0}>0$ tel que $\mathcal{L}+\varepsilon_{0}$ admette une sursolution locale strictement positive. On sait qu'alors $\mathcal{L}$ admet une fonction de Green $G$ vérifiant le principe de Harnack à l'infini suivant (ref. [An6], [An5]) : si $X, Y, Z \in M, Y \in[X, Z]$ et si $d(X, Y), d(Y, Z) \geq 1$ alors

$$
c^{-1} G(X, Y) G(Y, Z) \leq G(X, Z) \leq c G(X, Y) G(Y, Z)
$$

pour une constante $c=c(M) \geq 1$. De plus $c^{-1} \leq G(X, Y) \leq c$ pour $d(X, Y)=1$. Il s'ensuit (compte tenu de Harnack) que la fonction

$$
\tilde{G}(X, Y)=\mathbf{1}_{d(X, Y) \geq 1} G(X, Y)+\mathbf{1}_{d(X, Y)<1}
$$

vérifie la condition (PHI) du 9.B. Il est clair aussi que le (i) du 9.B est vérifié pour $C$ assez grand (propriété de Harnack pour $\mathcal{L}$ et son adjoint formel). Observons enfin la propriété suivante.

Lemme 9.3. Il existe des constantes strictement positives $c$ et $\alpha>0$ telles que

$$
\tilde{G}(X, Y) \tilde{G}(Y, X) \leq c e^{-\alpha d(X, Y)}
$$

pour $X, Y \in M$.

Preuve: On peut se limiter au cas où $d(X, Y) \geq 1$. Fixons $\varepsilon>0$ tel que $4 \varepsilon<\varepsilon_{0}$ et donc tel que $\mathcal{L}+4 \varepsilon$ soit encore faiblement coercif. Notons $G^{t}$ la fonction de Green de $\mathcal{L}+t I$. On sait [An5], [An6] que la faible coercivité et les hypothèses de géométrie bornée impliquent que $G\left(Z, Z^{\prime}\right) \leq$ $c e^{-\beta d\left(Z, Z^{\prime}\right)} G^{\varepsilon}\left(Z, Z^{\prime}\right)$ pour certaines constantes $c>0, \beta>0$ (et tout $\left.\left(Z, Z^{\prime}\right) \in M^{2}\right)$. Fixons $X^{\prime} \in M$ tel que $d\left(X^{\prime}, X\right)=\frac{1}{2}$. Comme $\mathcal{L}+2 \varepsilon$ est faiblement coercif $G^{2 \varepsilon}\left(X, X^{\prime}\right) \leq c$ pour une constante $c=c(\mathcal{L}, \varepsilon)$ ([An5, Proposition 7]). En utilisant l'équation résolvante, les inégalités 
de Harnack et les hypothèses de géométrie bornée on en déduit :

$$
G^{\varepsilon}(X, Y) G^{\varepsilon}\left(Y, X^{\prime}\right) \leq c G^{2 \varepsilon}\left(X, X^{\prime}\right) \leq c^{\prime}=c^{\prime}(\varepsilon, \mathcal{L}),
$$

et donc (avec Harnack)

$$
\tilde{G}(X, Y) \tilde{G}(Y, X) \leq c_{1}^{\prime} G(X, Y) G\left(Y, X^{\prime}\right) \leq c_{2}^{\prime} e^{-2 \beta d(X, Y)}
$$

pour de nouvelles constantes positives $c_{1}^{\prime}$ et $c_{2}^{\prime}$ ne dépendant que de $\mathcal{L}$.

Le lemme précédent assure en particulier que $\tilde{G}$ vérifie le (iii) du 9.B. Le théorème suivant résulte alors du 9.B et de [An8].

Théorème 9.4. Fixons $X_{0} \in M$ et posons $d_{\tilde{\theta}}(X, Y)=\frac{\tilde{G}\left(X, X_{0}\right) \tilde{G}\left(X_{0}, Y\right)}{\tilde{G}(X, Y)}$ pour $X, Y \in M$. Alors $d_{\tilde{\theta}}$ est quasi-symétrique et vérifie l'inégalité de quasi-métrique, i.e. il existe $C \geq 1$ tel que

$$
d_{\tilde{\theta}}(X, Z) \leq C \max \left\{d_{\tilde{\theta}}(X, Y), d_{\tilde{\theta}}(Y, Z)\right\}
$$

pour tout $(X, Y, Z) \in M^{3}$.

La quasi-symétrie du noyau $\tilde{\theta}=d_{\tilde{\theta}}^{-1}$ découle de (9.2) (cf. [An8]). L’inégalité de quasi-métrique découle du 9.C.1 et (9.4).

Un examen de cas, assez fastidieux et omis ici, permet de montrer par ailleurs l'inégalité de quasi-métrique (9.1) [i.e. pour l'inverse du noyau de Naïm $\theta$ ] pour les triplets $(X, Y, Z)$ tels que

$$
\min \left\{d(X, Y), d(Y, Z), d(X, Z), d\left(X, X_{0}\right), d\left(Y, X_{0}\right), d\left(Z, X_{0}\right)\right\} \leq 1 .
$$

Pour ces cas, on utilise uniquement le principe du maximum et les inégalités de Harnack à toutes les petites échelles (i.e. uniformément pour les boules $B(x, r), x \in M, r \leq 1)$. Avec le Théorème 9.4, ces vérifications donnent la propriété de quasi-métrique pour $d_{\theta}=\theta^{-1}$ en toute généralité.

3. Le cas des domaines intérieurement uniformes bornés. On suppose maintenant que $\Omega$ est un domaine borné et intérieurement uniforme dans $\mathbb{R}^{d}, d \geq 3$. Soit $x_{0} \in \Omega$.

3A. On va d'abord considérer le cas d'un opérateur elliptique d'ordre deux sur $\mathbb{R}^{d}$ de la forme $L(u)=\operatorname{div}(A(\nabla u))$ où $A(x)=\left\{a_{i j}(x)\right\}_{1 \leq i, j \leq d}$ est une matrice symétrique à coefficients mesurables uniformément bornés sur $\mathbb{R}^{d}$ telle que $\langle A(x) \xi, \xi\rangle \geq c_{0}\|\xi\|^{2}$ pour tout $\xi \in \mathbb{R}^{d}$ et une constante $c_{0}>0$ convenable. 
Munissons $\Omega$ de la métrique pseudo-hyperbolique $d_{h}$, i.e. $|d x|_{h}=$ $\frac{|d x|}{\delta(x)}$ pour $x \in \Omega$. D'après [BHK, Théorème 3.6$],\left(\Omega, d_{h}\right)$ est Gromovhyperbolique et tout segment géodésique de $\left(\Omega, d_{h}\right)$ est un arc intérieurement $c$-uniforme de $\Omega, c=c(\Omega)>0$ (ref. [BHK, p. 11]). D'autre part, si on pose $\tilde{G}(x, y)=\delta^{d-2}(y) G(x, y)$ si $d_{h}(x, y) \geq 1$ et $\tilde{G}(x, y)=1$ si $d_{h}(x, y)<1$, alors par le (PHI) du Corollaire 6.11 (dont on a dit qu'il s'étend à l'opérateur $L$ considéré ici, cf. le 7 ) $\tilde{G}$ vérifie les conditions énoncées en 9.B. On en déduit que l'inverse du pseudo noyau de Naïm $d_{\tilde{\theta}}(x, y)=\frac{\tilde{G}\left(x, x_{0}\right) G \tilde{G}\left(x_{0}, y\right)}{\tilde{G}(x, y)}$ est une pseudo-métrique.

3B. Si on suppose de plus $\Omega$ uniformément régulier (au sens de [An4]) on peut envisager des opérateurs elliptiques bien plus généraux. Ainsi si $L$ est de la forme $L u=\operatorname{div}(A \nabla u)+B \cdot \nabla u+V u$ avec $A$ comme en $\mathrm{C} 1$ et $B, V$ mesurables sur $\Omega$ tels que $\delta\|B\|+\delta^{2}|V| \leq C$ sur $\Omega$ et (pour alléger) $V \leq 0$, on sait que $\mathcal{L}=\delta^{2} L$ est un opérateur adapté et faiblement coercif sur $\left(\Omega, d_{h}\right)[\mathbf{A n} \mathbf{7}]$. Donc le B s'applique (ici on n'utilise pas les résultats de $[\mathbf{A i k}]$ ou ceux du $\S 6$, mais le principe de Harnack à l'infini de [An6]). Il s'ensuit que la fonction $d_{\tilde{\theta}}$ associée à la fonction de Green modifiée $\tilde{G}$ de $L$ est une quasi-métrique.

\section{Références}

[Aik] H. AIKAWA, Boundary Harnack principle and Martin boundary for a uniform domain, J. Math. Soc. Japan 53(1) (2001), 119-145.

[AHL] H. Aikawa, K. Hirata et T. Lundh, Martin boundary points of a John domain and unions of convex sets, J. Math. Soc. Japan 58(1) (2006), 247-274.

[ALM] H. Aikawa, T. Lundh ET T. Mizutani, Martin boundary of a fractal domain, Potential Anal. 18(4) (2003), 311-357.

[An1] A. AnConA, Principe de Harnack à la frontière et théorème de Fatou pour un opérateur elliptique dans un domaine lipschitzien, Ann. Inst. Fourier (Grenoble) 28(4) (1978), 169-213, x.

[An2] A. Ancona, Une propriété de la compactification de Martin d'un domaine euclidien, Ann. Inst. Fourier (Grenoble) 29(4) (1979), ix, 71-90.

[An3] A. Ancona, Régularité d'accès des bouts et frontière de Martin d'un domaine euclidien, J. Math. Pures Appl. (9) 63(2) (1984), $215-260$. 
[An4] A. Ancona, On strong barriers and an inequality of Hardy for domains in $\mathbb{R}^{n}$, J. London Math. Soc. (2) 34(2) (1986), $274-290$.

[An5] A. AnCONA, Negatively curved manifolds, elliptic operators, and the Martin boundary, Ann. of Math. (2) 125(3) (1987), $495-536$.

[An6] A. Ancona, Théorie du potentiel sur les graphes et les variétés, in : "École d'été de Probabilités de Saint-Flour XVIII-1988", Lecture Notes in Math. 1427, Springer, Berlin, 1990, pp. 1-112.

[An7] A. Ancona, First eigenvalues and comparison of Green's functions for elliptic operators on manifolds or domains, J. Anal. Math. 72 (1997), 45-92.

[An8] A. AnconA, Some results and examples about the behavior of harmonic functions and Green's functions with respect to second order elliptic operators, Nagoya Math. J. 165 (2002), $123-158$.

[An9] A. AnCONA, Quasimetric property for the inverse of Naïm's kernel, Manuscrit (1999), 4 p.

[AC] A. Ancona et N. Chevallier, Sur la convergence radiale des potentiels associés à l'équation de Helmholtz, Bull. Soc. Math. France 128(2) (2000), 249-281.

[BB] Z. M. Balogh et S. M. Buckley, Geometric characterizations of Gromov hyperbolicity, Invent. Math. 153(2) (2003), 261-301.

[BV1] Z. BAlOGH ET A. Volberg, Boundary Harnack principle for separated semihyperbolic repellers, harmonic measure applications, Rev. Mat. Iberoamericana 12(2) (1996), 299-336.

[BV2] Z. BALOGH ET A. VolberG, Geometric localization, uniformly John property and separated semihyperbolic dynamics, Ark. Mat. 34(1) (1996), 21-49.

[BBZ] R. F. BAss ET K. Burdzy, A boundary Harnack principle in twisted Hölder domains, Ann. of Math. (2) 134(2) (1991), $253-276$.

[BHK] M. Bonk, J. Heinonen et P. Koskela, Uniformizing Gromov hyperbolic spaces, Astérisque 270 (2001), viii+99 pp.

[BS] M. Bonk et O. Schramm, Embeddings of Gromov hyperbolic spaces, Geom. Funct. Anal. 10(2) (2000), 266-306. 
[Bou] N. Bourbaki, "Éléments de mathématique. I : Les structures fondamentales de l'analyse. Fascicule VIII. Livre III : Topologie générale. Chapitre 9 : Utilisation des nombres réels en topologie générale", Deuxième édition revue et augmentée, Actualités Scientifiques et Industrielles 1045, Hermann, Paris, 1958.

[Br1] M. Brelot, Le problème de Dirichlet "ramifié", Ann. Univ. Grenoble. Sect. Sci. Math. Phys. (N.S.) 22 (1946), 167-200.

[Br2] M. Brelot, Le problème de Dirichlet géodésique, C. R. Acad. Sci. Paris 228 (1949), 1790-1792.

[Br3] M. Brelot, Le problème de Dirichlet avec la frontière de Martin, C. R. Acad. Sci. Paris 240 (1955), 142-144.

[Br4] M. Brelot, "Axiomatique des fonctions harmoniques", Deuxième édition, Séminaire de Mathématiques Supérieures 14 (Été, 1965), Les Presses de l'Université de Montréal, Montreal, Que., 1969.

[BD] M. Brelot et J. L. Doob, Limites angulaires et limites fines, Ann. Inst. Fourier (Grenoble) 13, fasc. 2 (1963), 395-415.

[BSt] S. M. Buckley et A. Stanoyevitch, Weak slice conditions, product domains, and quasiconformal mappings, Rev. Mat. Iberoamericana 17(3) (2001), 607-642.

[CFMS] L. Caffarelli, E. Fabes, S. Mortola et S. Salsa, Boundary behavior of nonnegative solutions of elliptic operators in divergence form, Indiana Univ. Math. J. 30(4) (1981), 621-640.

[Car] L. CARLESON, On the existence of boundary values for harmonic functions in several variables, Ark. Mat. 4 (1962), 393-399.

[CM] M. Cranston et T. R. McConnell, The lifetime of conditioned Brownian motion, Z. Wahrsch. Verw. Gebiete 65(1) (1983), 1-11.

[Da1] B. E. J. DahlBerg, Estimates of harmonic measure, Arch. Rational Mech. Anal. 65(3) (1977), 275-288.

[Da2] B. E. J. DAhlBerG, On the existence of radial boundary values for functions subharmonic in a Lipschitz domain, Indiana Univ. Math. J. 27(3) (1978), 515-526.

[Doo] J. L. Doob, Some classical function theory theorems and their modern versions, Ann. Inst. Fourier (Grenoble) 15, fasc. 1 (1965), 113-136.

[Han] W. HANSEN, Uniform boundary Harnack principle and generalized triangle property, J. Funct. Anal. 226(2) (2005), 452-484. 
[Her] R.-M. HeRvé, Recherches axiomatiques sur la théorie des fonctions surharmoniques et du potentiel, Ann. Inst. Fourier (Grenoble) 12 (1962), 415-571.

[HW1] R. A. Hunt et R. L. Wheeden, On the boundary values of harmonic functions, Trans. Amer. Math. Soc. 132 (1968), $307-322$.

[HW2] R. A. Hunt et R. L. Wheeden, Positive harmonic functions on Lipschitz domains, Trans. Amer. Math. Soc. 147 (1970), $507-527$.

[JK] D. S. JeRISON ET C. E. KeniG, Boundary behavior of harmonic functions in nontangentially accessible domains, $A d v$. in Math. 46(1) (1982), 80-147.

[KV] N. J. Kalton et I. E. Verbitsky, Nonlinear equations and weighted norm inequalities, Trans. Amer. Math. Soc. 351(9) (1999), 3441-3497.

[Lit] J. E. Littlewood, On functions subharmonic in a circle. II, Proc. London Math. Soc. (2) 28 (1928), 383-394.

[Löm] A. Lömker, Martin boundaries of quasi-sectorial domains, Potential Anal. 13(1) (2000), 11-67.

[LMT] T. J. Lyons, K. B. MacGibbon et J. C. Taylor, Projection theorems for hitting probabilities and a theorem of Littlewood, J. Funct. Anal. 59(3) (1984), 470-489.

[Mar] R. S. MARTin, Minimal positive harmonic functions, Trans. Amer. Math. Soc. 49 (1941), 137-172.

[MS] O. MARTio ET J. SARvas, Injectivity theorems in plane and space, Ann. Acad. Sci. Fenn. Ser. A I Math. 4(2) (1979), $383-401$.

[Naï] L. NAÏM, Sur le rôle de la frontière de R. S. Martin dans la théorie du potentiel, Ann. Inst. Fourier, Grenoble 7 (1957), $183-281$.

[NV] R. NÄKki ET J. VÄIsÄLÄ, John disks, Exposition. Math. 9(1) (1991), 3-43.

[Tay] J.-C. TAYLOR, On the Martin compactification of a bounded Lipschitz domain in a Riemannian manifold, Ann. Inst. Fourier (Grenoble) 28(2) (1978), v, 25-52.

[Va1] J. VÄıs̈̈LÄ, Uniform domains, Tohoku Math. J. (2) 40(1) (1988), 101-118. 
[Va2] J. VÄıs̈̈L̈̈, Relatively and inner uniform domains, Conform. Geom. Dyn. 2 (1998), 56-88 (electronic).

[Va3] J. VÄISÄLÄ, Hyperbolic and uniform domains in Banach spaces, Ann. Acad. Sci. Fenn. Math. 30(2) (2005), 261-302.

[Zha] Shi Ying ZHAO, Boundary behavior of subharmonic functions in nontangential accessible domains, Studia Math. 108(1) (1994), 25-48.

Département de Mathématiques

Université Paris Sud

Campus d'Orsay, Bât. 425

91405 Orsay Cedex

France

E-mail address: alano.ancona@math.u-psud.fr

Primera versió rebuda el 13 de setembre de 2006, darrera versió rebuda el 14 de febrer de 2007. 